Aproximando ondas viajantes por equilíbrios de uma equação não local

\author{
Glauce Barbosa Verão
}

TESE APRESENTADA

$\mathrm{AO}$

Instituto DE MATEMÁticA E Estatística

DA

Universidade DE SÃo PAUlo

PARA

OBTENÇÃO DO TÍTULO

$\mathrm{DE}$

DOUTORA EM CIÊNCIAS

Programa: Matemática

Orientador: Prof. Dr. Luiz Augusto Fernandes de Oliveira

São Paulo, dezembro de 2016 


\section{Aproximando ondas viajantes por equilíbrios de uma equação não local}

Esta versão da tese contém as correções e alterações sugeridas pela Comissão Julgadora durante a defesa da versão original do trabalho, realizada em 02/12/2016. Uma cópia da versão original está disponível no

Instituto de Matemática e Estatística da Universidade de São Paulo.

Comissão Julgadora:

- Prof. Dr. Luiz Augusto Fernades de Oliveira (orientador) - IME-USP

- Prof. Dr. Antônio Luiz Pereira - IME-USP

- Prof ${ }^{a}$. Dra ${ }^{a}$ Gleiciane da Silva Aragão - UNIFESP

- Prof. Dr. Ma To Fu - ICMC - USP

- Prof ${ }^{a}$. Dra ${ }^{a}$. Rita de Cássia Dornelas Sodré Broche - UFLA 


\section{Agradecimentos}

Ao concluir este trabalho, agradeço:

A Nossa Senhora de Aparecida, por me proteger e me ajudar a passar por todos os momentos difíceis.

Ao meu orientador Luiz Augusto F. de Oliveira pela orientação e dedicação para tornar possível este trabalho.

Aos membros da banca examinadora, Rita, Gleiciane, Anôonio e Ma To Fu, que me ajudaram a enriquecer e melhorar este trabalho.

Aos meus pais Valdete e Erico, minha irmã Jaqueline que acreditaram no meu sonho e não mediram esforços para me ajudar a realizá-lo. Mãe, você que dividiu seu salário comigo para que eu pudesse permanecer em São Paulo estudando, dedico e devo tudo a você.

Ao Evandro que suportou todas as crises de choro e com muito carinho e paciência me ajudou a superar cada etapa.

Aos amigos que fiz durante o doutorado, Maikel, Vinícius, Ariadne, Itailma, Ânderson, Eliane, Oscar, Rosilene, Joelson, Hector e Adilson, vocês me ajudaram de diversas formas e tornaram essa caminhada mais leve e prazerosa.

Ao professor Cosme E. R. Mercedes da UEMS que me ajudou com a simulação numérica do Matlab e pela sua importância em toda minha trajetória acadêmica.

A Universidade São Judas Tadeu pela oportunidade de atuar como docente e me sentir realizada, a cada dia, com a profissão que sempre sonhei.

As professoras Delma Freo Faccin e Maristela Missio pelo incentivo e ajuda no início desta caminhada acadêmica 


\section{Resumo}

VERÃO, G. B. Aproximando ondas viajantes por equilíbrios de uma equação não local. 2016. 87 f. Tese (Doutorado) - Instituto de Matemática e Estatística, Universidade de São Paulo, São Paulo, 2016.

O sistema de FitzHugh-Nagumo possui um tipo especial de solução chamadas ondas viajantes, que são da forma $u(x, t)=\phi(x+c t)$ e $w(x, t)=\psi(x+c t)$ e além disso sabe-se que ela é estável. Tem-se o interesse de obter uma caracterização de seu perfil $(\phi, \psi)$ e sua velocidade de propagação c. Fazendo uma mudança de variáveis, transformamos tal problema em encontrar equilíbrios de uma equação não local. Esta equação não local possui uma onda viajante de velocidade zero cujo perfil é o mesmo da equação original e, com esta equação, é possível aproximar, ao mesmo tempo, o perfil e a velocidade da onda viajante. Como a intenção é usar métodos numéricos para aproximar tais soluções, o problema não local foi analisado em um intervalo limitado verificando a existência e algumas propriedades espectrais em domínios limitados.

Palavras-chave: FiztHugh-Nagumo, soluções ondas viajantes, equação não local. 


\section{Abstract}

VERÂO, G. B. Approximating traveling waves by equilibria of nonlocal equations . 2016. 87 f. Tese (Doutorado) - Instituto de Matemática e Estatística, Universidade de São Paulo, São Paulo, 2016.

The FitzHugh-Nagumo systems have a special kind of solution named traveling wave, which has a form $u(x, t)=\phi(x+c t)$ and $w(x, t)=\psi(x+c t)$ and furthermore it is a stable solution. It is our interest to obtain a characterization of its profile $(\phi, \psi)$ and speed of propagation $c$. Changing variables, we transform the problem of finding these solutions in the problem of finding an equilibria in a nonlocal equation. This nonlocal equation has a traveling wave with zero speed whose profile is the same of the original equation, and the nonlocal equation is used to approximate the profile and speed of the traveling wave at the same time. To use numerical methods for approximating such solutions, the nonlocal problem was analyzed in a finite interval to check that the existence and some spectral properties on bounded domains.

Palavras-chave: FiztHugh-Nagumo, traveling wave solutions, nonlocal equations. 


\section{Sumário}

$\begin{array}{ll}\text { Lista de Figuras } & \text { ix }\end{array}$

1 Teoria Básica $\quad 5$



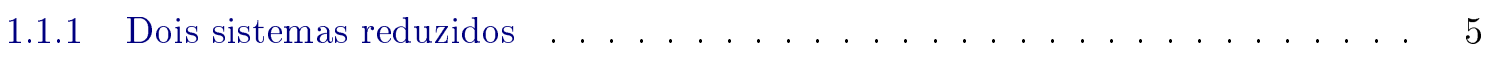

1.1.2 Demonstração da existência da onda viajante . . . . . . . . . . . . 6

1.1.3 Teoria geométrica da perturbação singular . . . . . . . . . . . . . . . 11

1.2 Estabilidade de ondas viajantes . . . . . . . . . . . . . . . . . 12

1.2 .1 Existência de Soluções . . . . . . . . . . . . . . . . . . . . . . . . . . . . . .

1.2.2 Função de Evans para sistema reduzido . . . . . . . . . . . . . . . 20

1.3 Função de Evans para sistema FitzHugh-Nagumo . . . . . . . . . . . . . . . . 21

1.4 Outros resultados - estimativas uniformes . . . . . . . . . . . . . 30

$\begin{array}{lll}2 & \text { Aproximando ondas viajantes } & \mathbf{3 7}\end{array}$

2.1 Definição do problema não local . . . . . . . . . . . . . . 37

2.2 Propriedades das soluções do problema local . . . . . . . . . . . . . . . . . . . . 39

2.3 Propriedades das soluções do problema não local . . . . . . . . . . . . . . . . . 41

3 Problema local em um intervalo limitado $\quad 47$

3.1 Existência e unicidade de soluções estacionárias . . . . . . . . . . . . . . . . . 47

3.2 Análise espectral do problema em intervalo finito . . . . . . . . . . . . 50

3.2 .1 Hipóteses e principais resultados . . . . . . . . . . . . . 50

3.2 .2 Aplicação no sistema de FitzHugh-Nagumo . . . . . . . . . . . . . 53

4 Problema não local em um intervalo limitado $\quad \mathbf{5 5}$

4.1 Soluções estacionárias do problema não local . . . . . . . . . . . . . . . 55

4.1.1 Convergência das soluções estacionárias para a onda viajante quando $|J| \rightarrow \infty \quad 56$



$4.2 .1 \quad$ Propriedades espectrais de $L^{r} \ldots \ldots \ldots \ldots \ldots \ldots \ldots$

4.2 .2 Propriedades espectrais de $L^{\infty} \ldots \ldots \ldots \ldots \ldots$

5 Convergência espectral $\quad 65$

5.1 Definições e resultados básicos . . . . . . . . . . . . . . . . . 65

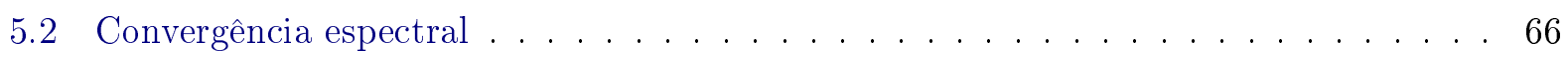


viii SUMÁRIO

6 Resultados Numéricos $\quad \mathbf{7 3}$

6.1 Definição da equação não local . . . . . . . . . . . . . . . . . . 73

6.2 Resultados numéricos . . . . . . . . . . . . . . . . . . . 73

$\begin{array}{ll}\text { Referências Bibliográficas } & 77\end{array}$ 


\section{Lista de Figuras}



2 Três pontos de equilíbrios de (1) para $\gamma>0$ suficientemente grande. . . . . . . . . 2

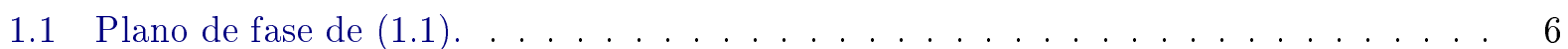



1.3 Intersecção das variedades $W^{u}(0,0,0, \bar{c})$ e $W^{s}(1,0,0, \bar{c}) \ldots \ldots \ldots \ldots \ldots \ldots$

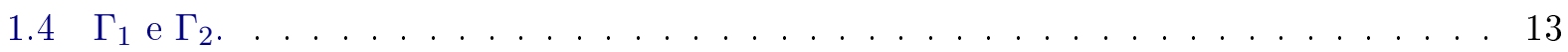

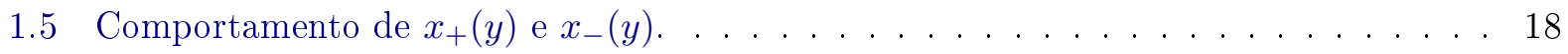

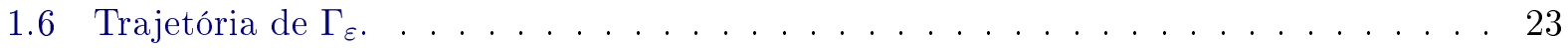

6.1 Velocidade de propagação $\lambda \ldots \ldots \ldots \ldots \ldots \ldots$. . . . . . . . . . . . . . . . . .

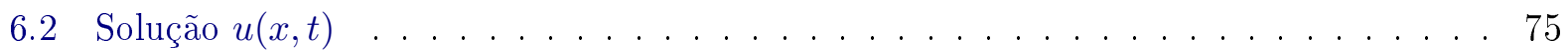

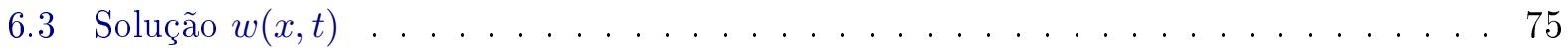




\section{Introdução}

O tema central de estudo deste trabalho está relacionado com a existência, estabilidade e aproximação de ondas viajantes do sistema de FitzHugh-Nagumo

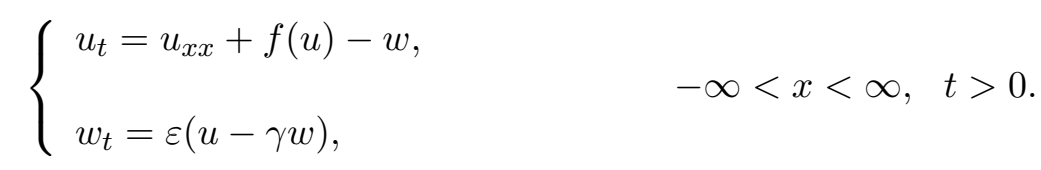

Em todo esse trabalho, $\varepsilon>0$ e $\gamma>0$ são constantes reais e $f: \mathbb{R} \rightarrow \mathbb{R}$ é uma função de classe $C^{2}$ satisfazendo as seguintes condições:

(H1) Existe $0<a<1$ tal que $f(0)=f(a)=f(1)=0, \quad f^{\prime}(0)<0, \quad f^{\prime}(1)<0$ e $f^{\prime}(a)>0$;

(H2) $f(u)>0$ quando $u<0$ ou $a<u<1$ e $f(u)<0$ quando $0<u<a$ ou $u>1$;

(H3) $\int_{0}^{1} f(s) d s>0$.

Uma representação de $f$ pode ser ilustrada na Figura 1.

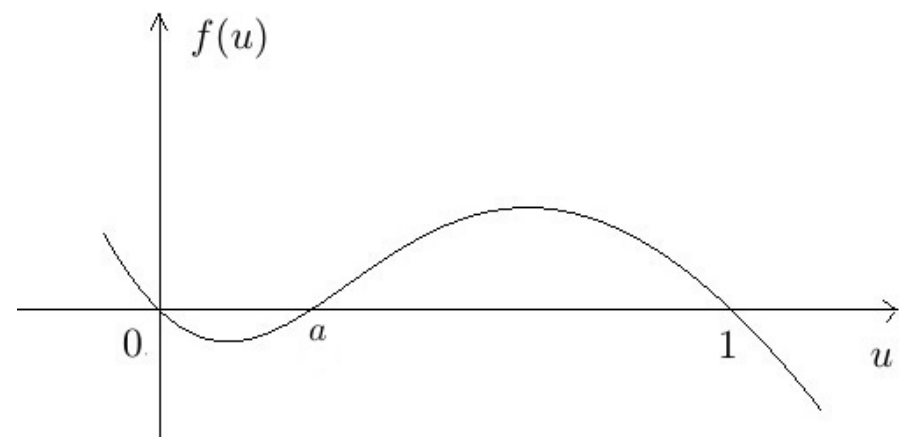

Figura 1: Função $f(u)$ satisfazendo as condições (H1)-(H3).

Um exemplo típico de uma função que satisfaz as hipóteses (H1)-(H3) é

$$
f(u)=u(u-a)(1-u),
$$

onde $0<a<\frac{1}{2}$.

Sistemas de reação difusão surgem em diversos processos biológicos como, por exemplo, a propagação dos estímulos do nervo axônio. Um dos trabalhos pioneiros nesta direção foi o artigo de Hodgkin e Huxley (1952). Mais tarde, FitzHugh (1961) e Nagumo et al. (1962) apresentaram uma versão simplificada do modelo de Hodgkin-Huxley e, desde então, é o objeto de estudo de diversos pesquisadores. Neste trabalho, vamos nos concentrar em um tipo especial de soluções chamadas ondas viajantes.

Ondas viajantes são soluções de (1) da forma

$$
u(x, t)=\phi(\xi), \quad w(x, t)=\psi(\xi) \quad \xi=x+c t
$$

onde $c>0$ é uma constante. Soluções de (1) da forma (3) são chamadas ondas viajantes de velocidade 
c e perfil $(\phi, \psi)$, ou simplesmente ondas viajantes de velocidade $c$. Naturalmente, se $\phi, \psi: \mathbb{R} \rightarrow \mathbb{R}$ são funções de classe $C^{2}$, então a terna $(\phi, \psi, c)$ é solução do sistema

$$
\left\{\begin{aligned}
\phi^{\prime \prime}-c \phi^{\prime}+f(\phi)-\psi & =0 \\
-c \psi^{\prime}+\varepsilon(\phi-\gamma \psi) & =0
\end{aligned}\right.
$$

Observemos que (4) é uma família de sistemas de equações diferenciais ordinárias dependendo do parâmetro $c$, que também é uma incógnita a ser determinada.

Vários autores estudaram existência de soluções de (4) para diversos valores de $c$ e demonstraram a existência de ondas viajantes de diversos perfis, tais como ondas periódicas e pulsos. Para uma exposição extensiva do assunto, recomendamos as referências: Jones (1984), Oliveira (1992) Szmolyan (1991) e Yanagida (1985).

Para descrevermos os perfis de ondas viajantes que consideraremos nessas notas, vamos examinar o sistema (4) mais detalhadamente. Primeiro, escrevemos (4) como o sistema de primeira ordem dependendo do parâmetro $c$ dado por

$$
\left\{\begin{aligned}
u^{\prime}(\xi) & =v(\xi) \\
v^{\prime}(\xi) & =c v(\xi)-f(u(\xi))+w(\xi) \\
w^{\prime}(\xi) & =\frac{\varepsilon}{c}(u(\xi)-\gamma w(\xi)) .
\end{aligned}\right.
$$

O número de pontos de equilíbrios de (5) depende apenas de $\gamma$. Durante todo este trabalho, tal parâmetro irá satisfazer a seguinte hipótese:

(H4) $\gamma>0$ é suficientemente grande para que $f(u)=\frac{1}{\gamma} u$ tenha três raízes reais $0<a<u_{1}<u_{2}<1$ $\mathrm{e}$

$$
\int_{0}^{u_{2}}\left(f(u)-\frac{1}{\gamma} u\right) d u>0
$$

como mostra a Figura 2.

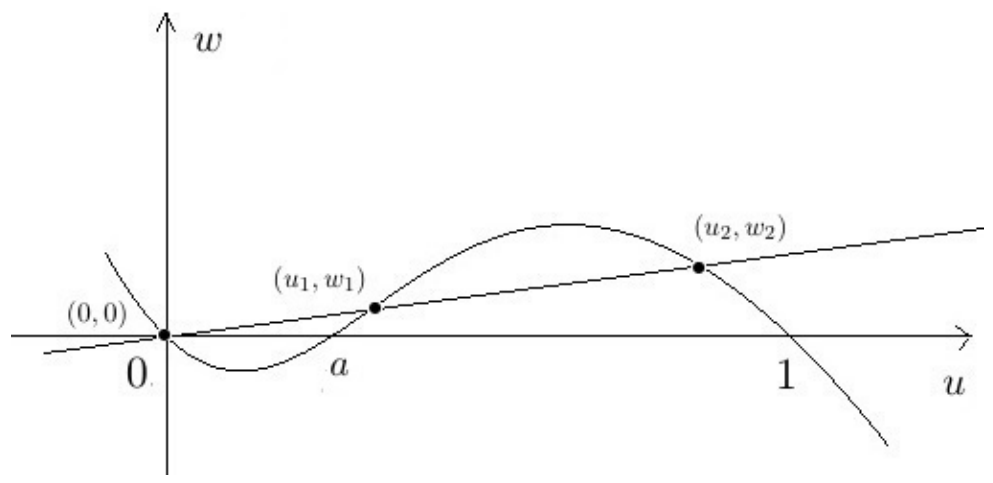

Figura 2: Três pontos de equilíbrios de (1) para $\gamma>0$ suficientemente grande.

Quando $f$ é a cúbica $f(u)=u(u-a)(1-u)$, a condição (H4) é satisfeita quando $0<a<\frac{1}{2}$ e $\gamma>\frac{9}{(2-a)(1-2 a)}$.

Sob as hipóteses (H1)-(H4), o sistema (5) tem três equilíbrios $p_{0}=(0,0,0), p_{1}=\left(u_{1}, 0, \frac{1}{\gamma} u_{1}\right)$ e $p_{2}=\left(u_{2}, 0, \frac{1}{\gamma} u_{2}\right)$.

Nesse trabalho vamos estudar a existência, estabilidade e aproximação de ondas viajantes $(\phi, \psi)$ : $\mathbb{R} \rightarrow \mathbb{R}^{2}$ do sistema de FitzHugh-Nagumo (1) que satisfazem

$$
\left(\phi(-\infty), \phi^{\prime}(-\infty), \psi(-\infty)\right)=(0,0,0) \quad \text { e } \quad\left(\phi(+\infty), \phi^{\prime}(+\infty), \psi(+\infty)\right)=\left(u_{2}, 0, \frac{1}{\gamma} u_{2}\right)
$$

Mostraremos que existe um valor $c>0$ tal que (1) tem uma onda viajante estável; além disso, 
essa onda é única, a menos de translação na variável de fase $\xi$. Em seguida, descreveremos um método para aproximação dessa onda como equilíbrio de um sistema não local.

Geometricamente, procurar ondas viajantes $(\phi, \psi, c)$ de (1) satisfazendo (6) é equivalente a determinar valores do parâmetro real $c$ para os quais o sistema (5) admite uma órbita heteroclínica conectando os equilíbrios $p_{0}$ e $p_{2}$.

Observe ainda que ondas viajantes $(\phi, \psi, c)$ de (1) podem ser olhadas como equilíbrios de um sistema parabólico num sistema de coordenadas móvel: se $(u, w)$ é uma solução de (1) e $\xi=x+c t$, então

$$
\bar{u}(\xi, t)=u(x, t) \text { e } \bar{w}(\xi, t)=w(x, t)
$$

satisfazem

$$
\left\{\begin{array}{l}
\bar{u}_{t}=\bar{u}_{\xi \xi}-c \bar{u}_{\xi}+f(\bar{u})-\bar{w} \\
\bar{w}_{t}=-c \bar{w}_{\xi}+\varepsilon(\bar{u}-\gamma \bar{w}) .
\end{array}\right.
$$

Nessas variáveis, $(\phi, \psi)$ é um ponto de equilíbrio de (8). Obviamente, como solução de um sistema autônomo

$$
\{(\phi(\cdot+k), \psi(\cdot+k)): k \in \mathbb{R}\}
$$

é uma família uniparamétrica de pontos de equilíbrios de (8).

Desta forma, o conceito de estabilidade da onda viajante é o conceito de estabilidade orbital, que discutiremos nos próximos capítulos. Nesse contexto, o estudo de estabilidade orbital de famílias de equilíbrios é de fundamental importância e resultados positivos nessa direção, que serão aqui utilizados, podem ser encontrados em Henry (1981), para sistemas parabólicos, e em Evans (1975), Yanagida (1989) e Jones (1984), para sistemas de FitzHugh-Nagumo.

Esta tese está organizada da seguinte maneira: o Capítulo 1 é dedicado a mostrar a existência e estabilidade da solução onda viajante mencionada acima e, além disso, mostraremos algumas estimativas que serão usadas mais adiante. No Capítulo 2 definimos a equação não local

$$
\left\{\begin{array}{l}
p_{t}=p_{x x}-\eta^{\prime}(t) p_{x}+f(p)-q \\
q_{t}=-\eta^{\prime}(t) q_{x}+\varepsilon(p-\gamma q)
\end{array} \quad-\infty<x<\infty, \quad t>0\right.
$$

e mostramos que este problema possui uma família de ondas viajantes com velocidade $c=0$ cujo perfil converge exponencialmente para o perfil da onda viajante encontrada no Capítulo 1 . Além disso, o termo não local $n^{\prime}(t)$, converge exponencialmente para a velocidade da onda viajante do problema original.

A premissa deste estudo é poder aproximar numericamente o perfil e a velocidade da onda viajante através dos equilíbrios do problema não local (9). Para usar métodos numéricos é necessário truncar o domínio e por isso, no Capítulo 4 analisamos o problema não local em um intervalo limitado com certas condições de contorno, isto é,

$$
\begin{cases}p_{t}=p_{x x}-\frac{A-\left\langle q, p_{x}\right\rangle_{L^{2}(J)}}{\left\|p_{x}\right\|_{L^{2}(J)}^{2}} p_{x}+f(p)-q, & \\ q_{t}=-\frac{A-\left\langle q, p_{x}\right\rangle_{L^{2}(J)}}{\left\|p_{x}\right\|_{L^{2}(J)}^{2}} q_{x}+\varepsilon(p-\gamma q), & \\ p(x, 0)=u_{0}(x) & q(x, 0)=w_{0}(x) \\ p\left(x_{-}, t\right)=0, \quad p\left(x_{+}, t\right)=u_{2} & \text { e } q\left(x_{-}, t\right)=0 .\end{cases}
$$

As condições de contorno acima foram escolhidas de forma a imitar o comportamento da onda viajante do problema original. 
Assim como o problema local e o não local, definidos em $\mathbb{R}$, estão intimamente ligados, o problema não local (10) herda algumas propriedades do problema local definido no mesmo domínio e, por isso, o Capítulo 3 é dedicado ao estudo da existência e estabilidade dos equilíbrios do problema

$$
\begin{cases}u_{t}=u_{x x}-c u_{x}+f(u)-w, & x \in, \quad t>0 \\ w_{t}=-c w_{x}+\varepsilon(u-\gamma w), & \\ u(x, 0)=u_{0}(x) \quad \text { e } & w(x, 0)=w_{0}(x) \\ u\left(x_{-}, t\right)=0, \quad u\left(x_{+}, t\right)=u_{2} & \text { e } w\left(x_{-}, t\right)=0,\end{cases}
$$

onde $A=\int_{0}^{u_{2}} f(s) d s$. A análise espectral do problema (10) é feita em duas etapas: no Capítulo 4 consideramos a linearização de (10) ao redor da solução estacionária encontrada no Capítulo 3 e estudamos as propriedades espectrais deste operador definido tanto em $\mathbb{R}$ quanto no intervalo $J$ finito; no Capítulo 5 estudamos a convergência espectral para um determinado conjunto e para um intervalo suficientemente grande. 


\section{Capítulo 1}

\section{Teoria Básica}

\subsection{Existência de ondas viajantes}

Nesta seção vamos mostrar existência de uma onda viajante de (1) satisfazendo (6). Como vimos, isso é equivalente a demonstrar a existência de um número real $c>0$ tal que (5) tem uma órbita heteroclínica conectando os equilíbrios $p_{0}$ e $p_{2}$. A estratégia que adotaremos será a de mostrar que existe um valor de $c$ tal que a variedade instável de $p_{0}$ intercepta a variedade estável de $p_{2}$. Para isso, na próxima subseção, vamos estudar inicialmente dois sistemas obtidos de (5), doravante chamados sistemas reduzidos.

\subsubsection{Dois sistemas reduzidos}

Lema 1.1.1 Suponha que $f$ satisfaz as hipóteses (H1)-(H3). Então, existe $c=\bar{c}>0$ tal que o sistema

$$
\left\{\begin{array}{l}
u^{\prime}=v \\
v^{\prime}=c v-f(u) \quad '=\frac{d}{d \xi}
\end{array}\right.
$$

tem uma solução heteroclínica $\left(\bar{\phi}(\xi), \bar{\phi}^{\prime}(\xi)\right)$ tal que $\bar{\phi}^{\prime}(\xi)>0$ para todo $\xi \in \mathbb{R} e$

$$
\lim _{\xi \rightarrow-\infty}\left(\bar{\phi}(\xi), \bar{\phi}^{\prime}(\xi)\right)=(0,0) \quad e \quad \lim _{\xi \rightarrow+\infty}\left(\bar{\phi}(\xi), \bar{\phi}^{\prime}(\xi)\right)=(1,0)
$$

Lema 1.1.2 Suponha que $f$ e $\gamma$ satisfazem as hipóteses (H1)-(H4). Então, existe $c=\underline{c}>0$ tal que o sistema

$$
\left\{\begin{array}{l}
u^{\prime}=v \\
v^{\prime}=c v-f(u)+\frac{1}{\gamma} u
\end{array}\right.
$$

tem uma solução heteroclínica $\left(\underline{\phi}(\xi), \underline{\phi^{\prime}}(\xi)\right)$ tal que $\underline{\phi}^{\prime}(\xi)>0$ para todo $\xi \in \mathbb{R} e$

$$
\lim _{\xi \rightarrow-\infty}\left(\underline{\phi}(\xi), \underline{\phi}^{\prime}(\xi)\right)=(0,0) \quad \text { e } \quad \lim _{\xi \rightarrow+\infty}\left(\underline{\phi}(\xi), \underline{\phi^{\prime}}(\xi)\right)=\left(u_{2}, 0\right) .
$$

A demonstração dos lemas acima é um estudo de plano de fase dos sistemas (1.1) e (1.3) que podem ser encontrados em Henry (1981) e Fife e McLeod (1977).

Observação. Como observado em Henry (1981), p. 130, quando $f(u)=u(u-a)(1-u)$ e $0<a<\frac{1}{2}$, temos a solução explícita de (1.1) $\bar{\phi}$ e sua velocidade $\bar{c}$, dadas por:

$$
\bar{c}=\sqrt{2}\left(\frac{1}{2}-a\right) \text { e } \bar{\phi}(\xi)=\frac{1}{1+\exp (-\xi / \sqrt{2})} .
$$


Com pequenas modificações, podemos obter também expressões explícitas para $\underline{c}$ e $\underline{\phi}$ :

$$
\underline{c}=\sqrt{2}\left(\frac{u_{2}}{2}-u_{1}\right) \quad \text { e } \quad \underline{\phi}(\xi)=\frac{u_{2}}{1+\exp \left(-\xi u_{2} / \sqrt{2}\right)} .
$$

A Figura 1.1 ilustra como as soluções de (1.1) se comportam de acordo com o parâmetro $c$. A partir dela podemos concluir que $\underline{c}<\bar{c}$.
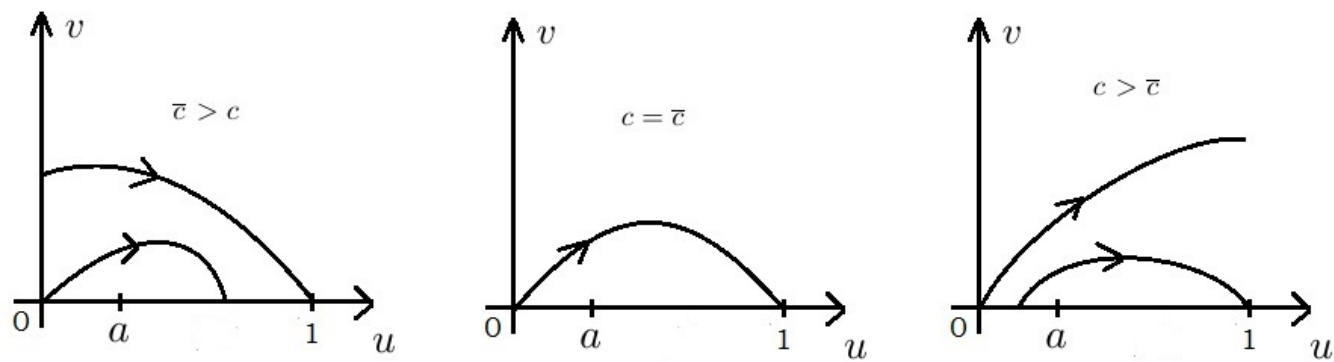

Figura 1.1: Plano de fase de (1.1).

\subsubsection{Demonstração da existência da onda viajante}

Voltemos agora nossa atenção ao sistema (5), para o qual queremos encontrar uma solução que conecta $p_{0}=(0,0,0) \operatorname{com} p_{2}=\left(u_{2}, 0, w_{2}\right)$, onde $w_{2}=\frac{1}{\gamma} u_{2}$.

A linearização de $(5)$ em torno de $p_{0}=(0,0,0)$ é a equação $Z^{\prime}=M_{0} Z$, onde $Z=(u, v, w)^{T}$ e

$$
M_{0}=\left(\begin{array}{ccc}
0 & 1 & 0 \\
-f^{\prime}(0) & c & 1 \\
\frac{\varepsilon}{c} & 0 & -\frac{\varepsilon \gamma}{c}
\end{array}\right) .
$$

Os autovalores de $M_{0}$ são as raízes do polinômio característico

$$
p(r)=-r^{3}+r^{2}\left(c-\frac{\varepsilon \gamma}{c}\right)+r\left(\varepsilon \gamma-f^{\prime}(0)\right)+\frac{\varepsilon}{c}-\frac{\varepsilon \gamma}{c} f^{\prime}(0) .
$$

Para cada $t \in \mathbb{R}$, temos

$$
p(i t)=\frac{1}{c}\left[t^{2}\left(\varepsilon \gamma-c^{2}\right)+\varepsilon\left(1-\gamma f^{\prime}(0)\right)\right]+i t\left[t^{2}+\varepsilon \gamma-f^{\prime}(0)\right] .
$$

Como $f^{\prime}(0)<0$, a equação $p(i t)=0$ não tem solução e, portanto, $p$ não tem raiz no eixo imaginário. Também, escrevendo $p$ como

$$
p(r)=\left(-\frac{\varepsilon \gamma}{c}-r\right)\left[r^{2}-c r+f^{\prime}(0)\right]+\frac{\varepsilon}{c},
$$

verificamos que se $\varepsilon>0$ é suficientemente pequeno, então $p(r)=0$ tem três raízes reais que satisfazem $r_{3}<r_{2}<0<r_{1}$. Portanto, para todos $c>0$ e $\varepsilon>0, M_{0}$ tem 3 autovalores que satisfazem:

$$
r_{3}<r_{2}<0<r_{1}
$$


Se $\vec{v}=\left(v_{1}, v_{2}, v_{3}\right)$ é um autovetor de $M_{0}$ associado a $r_{1}$, suas componentes satisfazem

$$
\left\{\begin{aligned}
v_{2} & =r_{1} v_{1} \\
-f^{\prime}(0) v_{1}+\left(c-r_{1}\right) v_{2}+v_{3} & =0 \\
\frac{\varepsilon}{\varepsilon \gamma+r_{1} c} v_{1} & =v_{3} .
\end{aligned}\right.
$$

Escolhendo $v_{1}=1$, concluímos que o espaço tangente à variedade instável em $(0,0,0)$ é gerado pelo vetor

$$
\vec{v}=\left(1, r_{1}, \frac{\varepsilon}{\varepsilon \gamma+c r_{1}}\right) .
$$

Para cada $c>0$, seja $(u(\xi), v(\xi), w(\xi))$ uma solução de (5) contida no primeiro octante que satisfaz

$$
\lim _{\xi \rightarrow-\infty}(u(\xi), v(\xi), w(\xi))=(0,0,0)
$$

e denote por $\Gamma_{c}$ a curva integral de (5) que entra na região $u>0, v>0$ e $w>0$ saindo de $(0,0,0)$ :

$$
\Gamma_{c}=\left\{(u(\xi), v(\xi), w(\xi)): \lim _{\xi \rightarrow-\infty}(u(\xi), v(\xi), w(\xi))=(0,0,0)\right\} .
$$

Da expressão (1.5) do autovetor $\vec{v}$ podemos selecionar $(u(0), v(0), w(0))$ em $\Gamma_{c}$ tão próximo de $(0,0,0)$ de modo que

$$
0<u(0)<a, \quad v(0)>0 \quad \text { e } \quad 0<w(0)<\frac{1}{\gamma} u(0) .
$$

Vamos procurar soluções de (5) que satisfaz (1.6) e também

$$
\lim _{\xi \rightarrow+\infty}(u(\xi), v(\xi), w(\xi))=\left(u_{2}, 0, w_{2}\right) .
$$

Lema 1.1.3 Para cada $c>0$, seja $\Gamma_{c}$ a curva integral de (5) saindo de $(0,0,0)$ e satisfazendo (1.7). Temos os seguintes resultados:

(i) $\Gamma_{c}$ não pode encontrar o plano $u=\gamma w$ em um ponto $\left(u\left(\xi_{0}\right), v\left(\xi_{0}\right), w\left(\xi_{0}\right)\right)$ com $u\left(\xi_{0}\right)>0$ se existe $\delta>0$ tal que $u(\xi)>0,0<w(\xi)<\frac{u(\xi)}{\gamma}$ e $v(\xi)>0$ para $\xi \in\left(\xi_{0}-\delta, \xi_{0}\right)$;

(ii) $\Gamma_{c}$ não pode interceptar o plano $w=0$ em um ponto $\left(u\left(\xi_{0}\right), v\left(\xi_{0}\right), w\left(\xi_{0}\right)\right)$ com $u\left(\xi_{0}\right)>0$ se existe $\delta>0$ tal que $u(\xi)>0$ e $v(\xi)>0$ para $\xi \in\left(\xi_{0}-\delta, \xi_{0}\right)$;

(iii) Se $\Gamma_{c}$ entra na região

$$
\Omega^{+}=\left\{(u, v, w): u>u_{2}, \quad v>0, \quad 0<w<\frac{1}{\gamma} u\right\}
$$

então $\Gamma_{c}$ permanece nesta região.

Demonstração. A demonstração é imediata e consiste em examinar a restrição do campo de vetores definido por (5) em cada um dos planos $w=0, w=\frac{1}{\gamma} u$ e $u=u_{2}$.

Um resultado que será muito usado nos próximos lemas é a comparação entre soluções de EDO cuja demonstração pode ser encontrada em Budincevic (2010).

Teorema 1.1.1 Considere os problemas

$$
\left\{\begin{array}{l}
y^{\prime}(x)=f(x, y) \\
y\left(x_{0}\right)=y_{0}
\end{array}\right.
$$


$e$

$$
\left\{\begin{array}{l}
z^{\prime}(x)=g(x, z) \\
z\left(x_{0}\right)=y_{0}
\end{array}\right.
$$

Suponha que (1.9) tem uma única solução em

$$
D=\left\{(x, y):\left|x-x_{0}\right|<\delta_{1}, \quad\left|y-y_{0}\right|<\delta_{2}\right\} .
$$

Se $f(x, y) \geq g(x, y)$ então $y_{0}(x) \geq z_{0}(x)$ para $x \geq x_{0}$ onde $y_{0}(x)$ é a única solução do problema (1.9) enquanto que $z_{0}(x)$ é a única solução do problema (1.10).

Sejam $\underline{c}$ e $\bar{c}$ os números obtidos nos Lemas 1.1.1 e 1.1.2.

Lema 1.1.4 Suponha que $f$ satisfaz as hipóteses $(H 1)-(H 4)$. Se $c \geq \bar{c}$, então $\Gamma_{c}$ é tal que $v(\xi)>0$ para todo $\xi \geq 0$.

Demonstração. Podemos escrever (1.4) na forma

$$
p(r)=\left(\frac{\varepsilon \gamma}{c}+r\right)\left(-r^{2}+c r-f^{\prime}(0)\right)+\frac{\varepsilon}{c} .
$$

Encontrar as raízes de $p$ é equivalente a encontrar $r$ que satisfaz

$$
-r^{2}+c r-f^{\prime}(0)+\frac{\varepsilon}{\varepsilon \gamma+c r}=0 .
$$

Portanto, o único autovalor positivo $r_{1}$ de $M_{0}$ satisfaz

$$
r_{1}=\frac{1}{2}\left\{c+\sqrt{c^{2}+4\left(\frac{\varepsilon}{\varepsilon \gamma+c r_{1}}-f^{\prime}(0)\right)}\right\} .
$$

Por outro lado, considerando a linearização do sistema

$$
\left\{\begin{array}{l}
u^{\prime}=v \\
v^{\prime}=\bar{c} v-f(u)
\end{array}\right.
$$

ao redor do equilíbrio $(0,0)$, obtemos o autovalor positivo

$$
r_{+}=\frac{1}{2}\left\{\bar{c}+\sqrt{\bar{c}^{2}-4 f^{\prime}(0)}\right\}
$$

e o seu correspondente autovetor $\overline{v_{1}}=\left(1, r_{+}\right)$. Como $c \geq \bar{c}$, então $r_{1}>r_{+}$.

Pelo Lema 1.1.1, (1.12) tem uma orbita heteroclínica $\left(\bar{\phi}(\xi), \bar{\phi}^{\prime}(\xi)\right)$ tal que $\bar{\phi}^{\prime}(\xi)>0$ e satisfaz (1.2). Podemos selecionar $\left(\bar{\phi}(0), \bar{\phi}^{\prime}(0)\right)$ na curva integral de (1.12) com valor inicial bem próximo de $(0,0)$.

Como $u^{\prime}(0)>0$ e $\bar{\phi}^{\prime}(0)>0$ podemos reescrever os problemas (5) e (1.12) nos respectivos problemas de valor inicial:

$$
\left\{\begin{array}{l}
\frac{d v}{d u}=\frac{c v-f(u)}{v}+\frac{w}{v} \\
\frac{d w}{d u}=\frac{\varepsilon(u-\gamma w)}{c v} \\
v(0)=0 \text { e } w(0)=0
\end{array}\right.
$$


$\mathrm{e}$

$$
\left\{\begin{array}{l}
\frac{d v}{d u}=\frac{\bar{c} v-f(u)}{v} \\
v(0)=0
\end{array}\right.
$$

Comparando as soluções dos problemas (1.13) e (1.14) concluímos que

$$
v(\xi) \geq \bar{\phi}^{\prime}(\xi)>0 \quad \text { para todo } \xi>0 .
$$

Lema 1.1.5 Se $c \leq \underline{c}$ então $\Gamma_{c}$ intersecta o plano $v=0$ em algum ponto $\left(u_{p}, 0, w_{p}\right)$ onde $u_{1} \leq$ $u_{p} \leq u_{2}$ e $0 \leq w_{p} \leq \frac{\overline{u_{p}}}{\gamma}$.

Demonstração. Pelo Lema 1.1.2 se $c=\underline{c}$ então existe uma órbita heteroclínica $\left(\underline{\phi}(\xi), \underline{\phi}^{\prime}(\xi)\right)$ ligando $(0,0)$ e $\left(u_{2}, 0\right)$.

Portanto o problema

$$
\left\{\begin{array}{l}
u^{\prime}=v, \\
v^{\prime}=c v-f(u)+\frac{1}{\gamma} u, \quad{ }^{\prime}=\frac{d}{d \xi} \\
w^{\prime}=\frac{\varepsilon}{c}(u-\gamma w) .
\end{array}\right.
$$

tem solução $\left(\underline{\phi}(\xi), \underline{\phi}^{\prime}(\xi), \underline{\psi}(\xi)\right)$ tal que

$$
\begin{aligned}
& \lim _{\xi \rightarrow-\infty}\left(\phi(\xi), \phi^{\prime}(\xi), \psi(\xi)\right)=(0,0,0) \\
& \lim _{\xi \rightarrow+\infty}\left(\phi(\xi), \phi^{\prime}(\xi), \psi(\xi)\right)=\left(u_{2}, 0, w_{2}\right)
\end{aligned}
$$

e $\underline{\phi}^{\prime}>0$ em $(-\infty, \infty)$.

Como feito no Lema 1.1.4, considere os problemas

$$
\left\{\begin{array}{l}
\frac{d v}{d u}=\frac{\underline{c} v-f(u)+\frac{u}{\gamma}}{v} \\
v(0)=0
\end{array}\right.
$$

e

$$
\left\{\begin{array}{l}
\frac{d v}{d u}=\frac{c v-f(u)+\frac{u}{\gamma}}{v}+\frac{w-\frac{u}{\gamma}}{v} \\
\frac{d w}{d u}=\frac{\varepsilon(u-\gamma w)}{c v} \\
v(0)=0 \text { e } w(0)=0 .
\end{array}\right.
$$

Pelo item (i) do Lema 1.1.3, $w<\frac{u}{\gamma}$. Comparando as soluções de (1.16) e (1.17) concluímos que a componente $v$ da solução de (1.17) satisfaz

$$
v(\xi) \leq \underline{\phi}^{\prime}(\xi), \quad \text { para todo } \xi>0 .
$$


Lema 1.1.6 Para qualquer $u_{+}>u(0)$ tal que

$$
F(u)=\int_{0}^{u}\left(f(s)-\frac{s}{\gamma}\right) d s>0 \quad \text { para } u \in\left(0, u_{+}\right),
$$

existe $\xi_{+}$, tal que $u\left(\xi_{+}\right)=u_{+}$e $u(\xi)>0, u(\xi)-\gamma w(\xi)>0, v(\xi)>0$ para $\xi \in\left(-\infty, \xi_{+}\right]$.

Demonstração. Veja Gao e Wang (2004), p.671 .

Teorema 1.1.2 Sob as hipóteses (H1)-(H4), para todo $\varepsilon>0$, existe $c^{*} \in(\underline{c}, \bar{c})$ tal que existe uma soluçấo onda viajante do problema (1) com velocidade $c^{*}$ satisfazendo (6), isto é, existe um único $c^{*} \in(\underline{c}, \bar{c})$ tal que existe uma única, a menos de translação, solução do problema (5)-(6).

Demonstração. Suponha que $\Gamma_{c}$ começa em um ponto na região

$$
R=\left\{(u, v, w) ; 0<u<u_{2}, 0<w<\max \left\{f(u), \frac{u}{\gamma}\right\}, v>0\right\}
$$

em $\xi=0$.

Pelo Lema 1.1.4, se $c \geq \bar{c}, \Gamma_{c}$ não intersecta com o plano $v=0$ para qualquer $\xi>0$ e portanto $\Gamma_{c}$ entrará na região

$$
S=\left\{(u, v, w) ; u>u_{2}, \max \{f(u), 0\}<w<\frac{u}{\gamma}, v>0\right\} .
$$

Pelo Lema 1.1.5, se $c=\underline{c}, \Gamma_{c}$ intersecta com $v=0$ em algum ponto $\left(u_{p}, 0, w_{p}\right)$ tal que $u_{1} \leq$ $u_{p} \leq u_{2}$ e $0 \leq w_{p} \leq \frac{u_{p}}{\gamma}$.

Logo, se $c=\underline{c}$ ou $\Gamma_{c}$ entra na região

$$
T=\left\{(u, v, w) ; u_{2}<u<1, w<f(u), v<0\right\} .
$$

ou tende para $\left(u_{2}, 0, w_{2}\right)$ quando $\xi \rightarrow+\infty$.

Suponha que $\Gamma_{c}$ entra na região $T$ e sejam

$$
\bar{C}=\left\{c>0, \exists \xi_{0} \text { tal que } \Gamma_{c} \text { está em R se } \xi \in\left(-\infty, \xi_{0}\right) \text { e entra em } \mathrm{S} \text { em } \xi=\xi_{0}\right\}
$$

$\mathrm{e}$

$$
\underline{C}=\left\{c>0, \exists \xi_{0} \text { tal que } \Gamma_{c} \text { está em R se } \xi \in\left(-\infty, \xi_{0}\right) \text { e entra em T em } \xi=\xi_{0}\right\} .
$$

$\bar{C}$ e $\underline{C}$ são conjuntos abertos, não vazios e além disso $\bar{C} \cap \underline{C}=\emptyset$. Podemos escrever

$$
\underline{C}=\left(c_{1}, c_{2}\right) \cup\left(c_{3}, c_{4}\right) \cup \cdots \cup\left(c_{i}, c_{i+1}\right) \cup \cdots
$$

onde $0<c_{1}<c_{2}<\cdots<c_{i}<\cdots<\bar{c}$.

Suponha que $\underline{c} \in\left(c_{j-1}, c_{j}\right)$ então $\underline{c}<c_{j}$ e $c_{j}$ pertence a fronteira de $\underline{C}$. Como $\Gamma_{c_{j}}$ não entra em $T$, existem dois casos a serem considerados:

Caso 1: $\Gamma_{c_{j}}$ permanece na região R. Como $u^{\prime}=v>0$ e que $u \in\left(0, u_{2}\right)$ é limitado então ou $\lim _{\xi \rightarrow+\infty}(u, v, w)=\left(u_{1}, 0, w_{1}\right)$ ou $\lim _{\xi \rightarrow+\infty}(u, v, w)=\left(u_{2}, 0, w_{2}\right)$. Pelo Lema 1.1.6 concluímos que $\lim _{\xi \rightarrow+\infty}(u, v, w)=\left(u_{2}, 0, w_{2}\right)$.

Caso 2: $\Gamma_{c_{j}}$ sai da região R. Como $\Gamma_{c_{j}}$ tem dependência contínua relativa a $c, \Gamma_{c_{j}}$ irá passar por algum ponto do contorno de $\mathrm{S}$ em algum tempo finito $\xi=\xi_{1} . \Gamma_{c_{j}}$ irá passar por algum ponto $\left(u\left(\xi_{1}\right), v\left(\xi_{1}\right), w\left(\xi_{1}\right)\right)$ 


$$
B=\left\{(u, v, w) ; u_{2} \leq u \leq 1, w=f(u), v=0\right\}
$$

Temos $u^{\prime}\left(\xi_{1}\right)=v\left(\xi_{1}\right)=0, \quad w^{\prime}\left(\xi_{1}\right)=\frac{\varepsilon}{c}\left(u\left(\xi_{1}\right)-\gamma w\left(\xi_{1}\right)\right)>0, \quad u^{\prime \prime}\left(\xi_{1}\right)=v^{\prime}\left(\xi_{1}\right)=0$.

$$
\begin{aligned}
u^{\prime \prime \prime}\left(\xi_{1}\right)=v^{\prime \prime}\left(\xi_{1}\right) & =\frac{d}{d u} f\left(u\left(\xi_{1}\right)\right) v\left(\xi_{1}\right)+w^{\prime}\left(\xi_{1}\right)+c v^{\prime}\left(\xi_{1}\right) \\
& =\frac{\varepsilon}{c}\left(u\left(\xi_{1}\right)-\gamma w\left(\xi_{1}\right)\right)>0 .
\end{aligned}
$$

Então $\Gamma_{c_{j}}$ entrará na região S e $c_{j} \in \bar{C}$. Como $\bar{C}$ é aberto, então existe $\delta>0$ tal que $\left(c_{j}-\delta, c_{j}+\right.$ $\delta) \subset \bar{C} \cup \underline{C}$ o que contradiz $\bar{C} \cup \underline{C}=\emptyset$.

\subsubsection{Teoria geométrica da perturbação singular}

A existência de ondas viajantes para equação de FitzHugh-Nagumo foi estudada e demonstrada de diferentes formas. Uma dessas formas é a Teoria Geométrica da Perturbação Singular (GPS) que de forma sucinta, vamos descrever os passos de sua demonstração para o caso da órbita heteroclínica.

Quando $\varepsilon=0$ no sistema

$$
\left\{\begin{array}{l}
u^{\prime}=v, \\
v^{\prime}=c v-f(u)+w, \quad{ }^{\prime}=\frac{d}{d \xi} \\
w^{\prime}=\frac{\varepsilon}{c}(u-\gamma w), \\
c^{\prime}=0,
\end{array}\right.
$$

então $w^{\prime}=0$ e portanto $w$ atua como um parâmetro no sistema

$$
\left\{\begin{array}{l}
u^{\prime}=v \\
v^{\prime}=c v-f(u)+w \\
w^{\prime}=0 \\
c^{\prime}=0
\end{array}\right.
$$

O sistema (1.19) tem os pontos de equilíbrios no conjunto

$$
\mathcal{S}=\{(u, v, w, c) ; v=0 \text { e } w=f(u)\} .
$$

Definem-se duas curvas $\mathcal{S}_{L}$ e $\mathcal{S}_{R}$ contidas em $\mathcal{S}$ onde $f^{\prime}(u)<0$ como mostra a Figura 1.2 para um valor de $c$ fixo.

Como $\mathcal{S}_{L}$ e $\mathcal{S}_{R}$ foram definidas longe dos pontos onde $f^{\prime}(u)=0$, os pontos $(u, 0, f(u), c)$ de $\mathcal{S}_{L}$ e $\mathcal{S}_{R}$ são pontos fixos hiperbólicos e definem uma variedade estável $W^{s}$ e uma variedade instável $W^{u}$.

Como existe $\bar{c}$ tal que, existe uma órbita no plano $w \equiv 0$ saindo de $(0,0)$ chegando em $(1,0)$ (Lema 1.1.1), isto significa que as variedades $W^{u}(0,0,0, \bar{c})$ e $W^{s}(1,0,0, \bar{c})$ se intersectam como ilustra a Figura 1.3.

Seja $\delta>0$ suficientemente pequeno e $I_{\delta}=[\bar{c}-\delta, \bar{c}+\delta]$, vamos denotar por

$$
\mathcal{N}^{u}=\bigcup_{c \in I_{\delta}} W^{u}(0,0,0, c) \quad \text { e } \quad \mathcal{N}^{s}=\bigcup_{c \in I_{\delta}} W^{s}\left(u_{2}, 0, f\left(u_{2}\right), c\right) .
$$




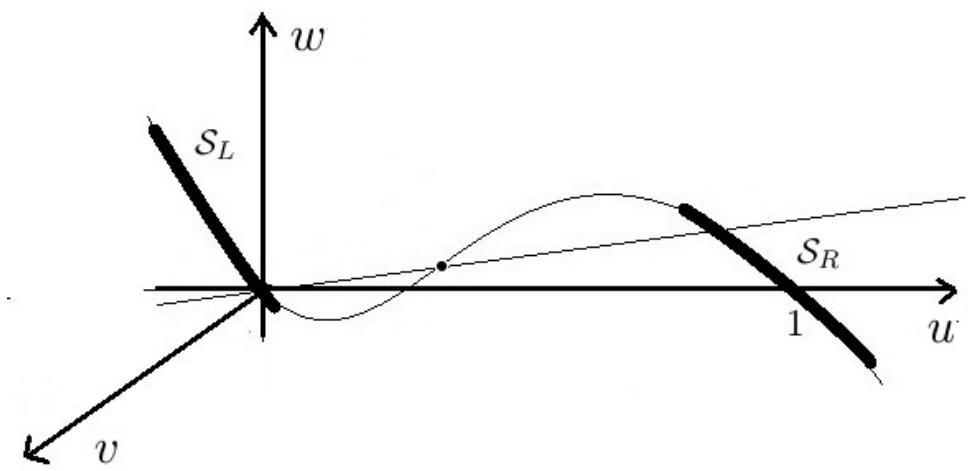

Figura 1.2: Curvas $\mathcal{S}_{L}$ e $\mathcal{S}_{R}$ para c fixo.



Figura 1.3: Intersecção das variedades $W^{u}(0,0,0, \bar{c})$ e $W^{s}(1,0,0, \bar{c})$.

Como existe uma órbita heteroclínica $\left(\phi, \phi^{\prime}, 0, \bar{c}\right)$ ligando os pontos $(0,0,0, \bar{c}) \in \mathcal{N}^{u}$ e $(1,0,0, \bar{c}) \in$ $\mathcal{N}^{s}$ isto implica que as variedades $\mathcal{N}^{u}$ e $\mathcal{N}^{s}$ se intersectam. O Teorema 4.1 de Szmolyan (1991) mostra que esta intersecção é transversal.

Para mais detalhes desta demonstração veja os artigos de Szmolyan (1991) e Jones (1995).

Seja $\Gamma_{0}=\Gamma_{1} \cup \Gamma_{2}$ a curva suave por partes, onde $\Gamma_{1}$ é a trajetória de $\bar{\phi}$ do Lema 1.1.1 e $\Gamma_{2}$ é o gráfico de $f(u)$ para $u \in\left[u_{2}, 1\right]$ como mostra a Figura 1.4

Yanagida (1989) usou os argumentos do GPS para enunciar o seguinte resultado

Teorema 1.1.3 Se $\varepsilon>0$ for suficientemente pequeno, (1) tem uma solução onda viajante cuja órbita $\Gamma_{\varepsilon}$ satisfaz; $\Gamma_{\varepsilon} \rightarrow \Gamma_{0}$ e sua velocidade de propagação $c^{*} \rightarrow \bar{c}$, quando $\varepsilon \rightarrow 0$.

\subsection{Estabilidade de ondas viajantes}

Nesta seção, vamos discutir a estabilidade da onda viajante

$$
\bar{u}(x, t)=\phi\left(x+c^{*} t\right), \quad \bar{w}(x, t)=\psi\left(x+c^{*} t\right),
$$

de (1), que satisfaz as condições

$$
\left(\phi(-\infty), \phi^{\prime}(-\infty), \psi(-\infty)\right)=(0,0,0) \quad \text { e } \quad\left(\phi(+\infty), \phi^{\prime}(+\infty), \psi(+\infty)\right)=\left(u_{2}, 0, \frac{1}{\gamma} u_{2}\right),
$$




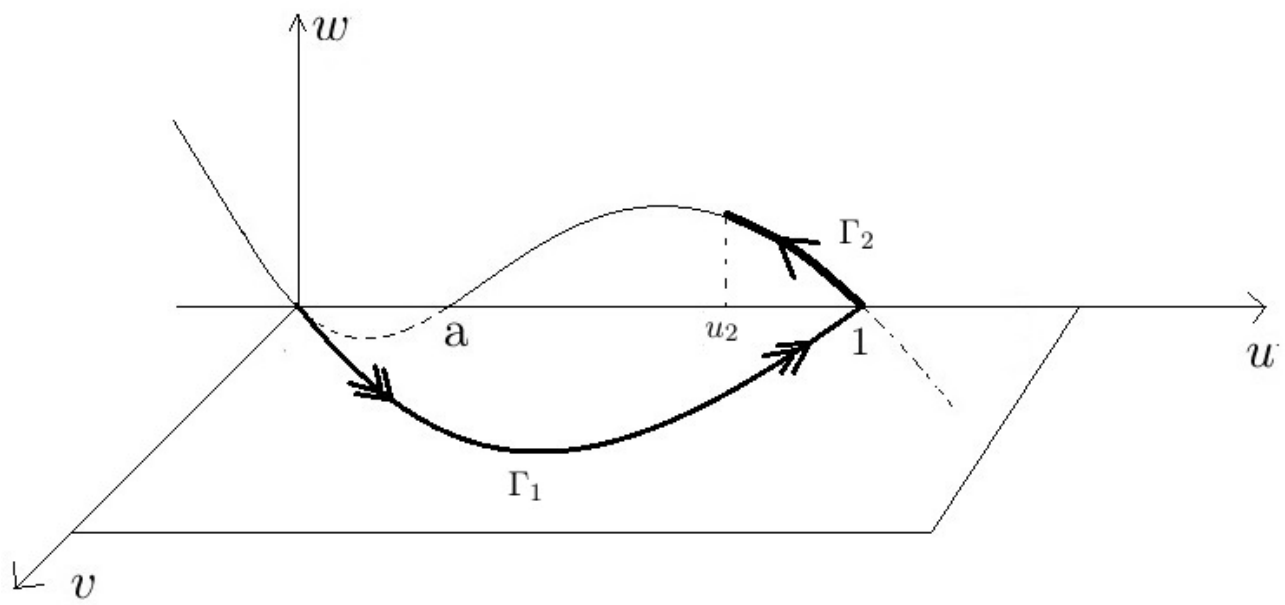

Figura 1.4: $\Gamma_{1} e \Gamma_{2}$.

obtida no Teorema 1.1.2.

Como observamos na introdução, a onda viajante $\left(\phi, \psi, c^{*}\right)$ de (1) pode ser olhada como equilíbrio de um sistema evolutivo num sistema de coordenadas móvel: se $(u, w)$ é uma solução de (1) e $\xi=x+c^{*} t$, então $\bar{u}(\xi, t)=u(x, t)$ e $\bar{w}(\xi, t)=w(x, t)$ satisfazem

$$
\left\{\begin{array}{l}
\bar{u}_{t}=\bar{u}_{\xi \xi}-c^{*} \bar{u}_{\xi}+f(\bar{u})-\bar{w} \\
\bar{w}_{t}=-c^{*} \bar{w}_{\xi}+\varepsilon(\bar{u}-\gamma \bar{w}) .
\end{array}\right.
$$

Nessas variáveis, $(\phi, \psi)$ é um ponto de equilíbrio de (1.23). Obviamente, como solução de um sistema autonômo (5), translações no "tempo" $\xi$ também são soluções, de modo que $\{(\phi(\cdot+k), \psi(\cdot+$ $k)): k \in \mathbb{R}\}$ é uma família uniparamétrica de pontos de equilíbrios de (1.23).

Desta forma, o conceito de estabilidade da onda viajante é o conceito de estabilidade orbital, que discutiremos neste capítulo. Naturalmente, precisamos eleger um espaço de fase $X$ e examinar (1.23) como um sistema de evolução em $X$. Na próxima subseção, faremos uma breve discussão desses assuntos, já que a literatura a respeito da boa colocação de (1) é bastante conhecida.

\subsubsection{Existência de Soluções}

Nessa seção trataremos de modo sucinto a teoria básica de soluções de (1.23). Seguiremos de perto o tratamento dado por Bates e Jones (1989). Detalhes podem também ser encontrados nas referências Evans (1972), Smoller (1983) e Rauch e Smoller (1978)

Em primeiro lugar, diversos espaços podem ser escolhidos para formular (1.23) como uma equação de evolução, mas vamos focar nos seguintes espaços de Banach:

1) $C_{\text {unif }}(\mathbb{R})=\{u: \mathbb{R} \rightarrow \mathbb{R}: u$ é limitada e uniformemente contínua $\}$ com a norma

$$
\|u\|_{\infty}=\sup \{|u(x)|:-\infty<x<\infty\} ;
$$

2) $W^{m, p}(\mathbb{R})=\left\{u: \mathbb{R} \rightarrow \mathbb{R}: u, u^{\prime}, \ldots, u^{(m)} \in L^{p}(\mathbb{R})\right\}^{1}, m \in \mathcal{Z}_{+}^{*}$ com a norma

$$
\|u\|_{m, p}=\left\{\int_{-\infty}^{\infty}\left(|u(x)|^{p}+\ldots+\left|u^{(m)}(x)\right|^{p}\right) d x\right\}^{1 / p} .
$$

\footnotetext{
${ }^{1}$ Derivadas no sentido de distribuição
} 
Nesse último caso, admitimos que $1<p<\infty$. Um fato básico que usaremos sistematicamente é o seguinte

Teorema 1.2.1 Para todo $1<p<\infty, W^{1, p}(\mathbb{R})$ e $C_{\text {unif }}(\mathbb{R})$ são espaços de Banach e $W^{1, p}(\mathbb{R}) \subset$ $C_{\text {unif }}(\mathbb{R})$ com inclusão contínua:

$$
\sup _{-\infty<x<\infty}|u(x)| \leq c\left\{\int_{-\infty}^{\infty}\left(|u(x)|^{p}+\left|u^{\prime}(x)\right|^{p}\right) d x\right\}^{1 / p},
$$

para toda $u \in W^{1, p}(\mathbb{R})$, com $c>0$. Em particular, $H^{1}(\mathbb{R}):=W^{1,2}(\mathbb{R})$ é um espaço de Hilbert.

Demonstração. Pode ser encontrada em Brezis (2010). forma

Seja $X$ qualquer um dos espaços $X=C_{\text {unif }}(\mathbb{R})^{2}$ ou $X=H^{1}(\mathbb{R})^{2}$. Tomando perturbação da

$$
\bar{u}(\xi, t)=\phi(\xi)+u(\xi, t), \quad \bar{w}(\xi, t)=\psi(\xi)+w(\xi, t),
$$

$\operatorname{com}(u(\cdot, t), w(\cdot, t)) \in X$, então $(u, w)$ satisfaz

$$
\begin{cases}u_{t}=u_{\xi \xi} & -c^{*} u_{\xi}+f^{\prime}(\phi(\xi)) u-w+f(\phi(\xi)+u)-f(\phi(\xi))-f^{\prime}(\phi(\xi)) u \\ w_{t}= & -c^{*} w_{\xi}+\varepsilon(u-\gamma w) .\end{cases}
$$

Defina os seguintes operadores em $X$ :

$A=\left[\begin{array}{cc}\frac{d^{2}}{d \xi^{2}} & 0 \\ 0 & 0\end{array}\right], \quad B=\left[\begin{array}{cc}-c^{*} \frac{d}{d \xi} & 0 \\ 0 & -c^{*} \frac{d}{d \xi}\end{array}\right], \quad C=\left[\begin{array}{cc}f^{\prime}(0) & -1 \\ \varepsilon & -\varepsilon \gamma\end{array}\right] \quad$ e $K=\left[\begin{array}{cc}f^{\prime}(\phi(\xi))-f^{\prime}(0) & 0 \\ 0 & 0\end{array}\right]$

então, as equações (1.25) podem ser escritas como o sistema de evolução

$$
\frac{d}{d t}\left(\begin{array}{c}
u \\
w
\end{array}\right)=(A+B+C+K)\left(\begin{array}{c}
u \\
w
\end{array}\right)+\left(\begin{array}{c}
f(\phi(\xi)+u)-f(\phi(\xi))-f^{\prime}(\phi(\xi)) u \\
0
\end{array}\right) .
$$

Agora, $-A$ é setorial e, portanto, $A$ gera um semigrupo analítico em $X$. Como $C$ é um operador limitado, então $A+C$ é o gerador de um semigrupo analítico $P(t)$ em $X$. Também, para $a \in \mathbb{R}$, o operador translação $T_{a}: X \rightarrow X$ dado por $T_{a}(u, w)(\xi)=(u(\xi+a), w(\xi+a))$ é um operador limitado (isometria) e é o $C^{0}$-grupo gerado por $B$. Um cálculo explícito mostra então que $A+B+C$ gera $T_{c^{*} t} P(t)$, que é um $C^{0}$-semigrupo em $X$. Finalmente, como $K$ é um operador limitado, $A+B+C+K$ é o gerador de um $C^{0}$-semigrupo em $X$.

Como $f$ é de classe $C^{2}$, a aplicação $F: X \rightarrow X$ dada por

$$
F(u, w)(\xi)=\left(f(\phi(\xi)+u(\xi))-f(\phi(\xi))-f^{\prime}(\phi(\xi)) u(\xi), 0\right)
$$

é de classe $C^{1}$, no sentido de Frechet, e portanto, o problema de Cauchy para (1.25) tem solução fraca local única. Existência global pode ser também ser obtida e recomendamos as referências Rothe (1984), Rauch (1976) Bates e Jones (1989), Pazy (2012).

Definição 1.2.1 Dizemos que a solução onda viajante $(\phi, \psi)$ de $(1.23)$ é estável se, dado $\epsilon>$ 0 , existe $\delta>0$ tal que $\left\|\left(u_{0}, w_{0}\right)\right\|<\delta$, então a soluçẫo $(\bar{u}(\cdot, t), \bar{w}(\cdot, t))$ de (1.23) satisfazendo $(\bar{u}(\cdot, 0), \bar{w}(\cdot, 0))=\left(\phi+u_{0}, \psi+w_{0}\right)$ satisfaz $\|(u(\cdot, t), w(\cdot, t))\|<\epsilon$, para todo $t \geq 0$.

Dizemos que a solução onda viajante $(\phi, \psi)$ de (1.23) é orbitalmente estável se ela é estável e existe $\rho>0$ tal que se $\left\|\left(u_{0}, w_{0}\right)\right\|<\rho$, então existe $\xi_{0} \in \mathbb{R}$ tal que a solução $(\bar{u}(\cdot, t), \bar{w}(\cdot, t))$ de (1.23) satisfaz

$$
\lim _{t \rightarrow \infty}\left[\left\|\bar{u}(\xi, t)-\phi\left(\xi-\xi_{0}\right)\right\|+\left\|\bar{w}(\xi, t)-\psi\left(\xi-\xi_{0}\right)\right\|\right]=0 .
$$


Como $\left\|f(\phi(\xi)+u)-f(\phi(\xi))-f^{\prime}(\phi(\xi)) u\right\| /\|u\| \rightarrow 0$ quando $\|u\| \rightarrow 0$, a equação linearizada da equação (1.23) ao redor de $(\phi, \psi)$ é dada por

$$
\begin{cases}u_{t}=u_{\xi \xi} & -c^{*} u_{\xi}+f^{\prime}(\phi(\xi)) u-w \\ w_{t}= & -c^{*} w_{\xi}+\varepsilon(u-\gamma w)\end{cases}
$$

Vamos agora fazer algumas observações importantes a respeito do resultado "estabilidade via linearização implica estabilidade da equação não linear". Devido ao caráter 'hiperbólico' da segunda equação em (1.27), os resultados de Henry (1981) não podem ser aplicados imediatamente. Evans (1972) demonstrou que a estabilidade da equação linearizada implica na estabilidade orbital da equação não linear para uma classe de equações que incluem o sistema de FitzHugh-Nagumo no caso de ondas homoclínicas. A conclusão foi mais tarde generalizada por Bates e Jones (1989). No presente contexto (de ondas heteroclínicas), o resultado é de fato também verdadeiro e, segundo nosso conhecimento, decorrem dos recentes resultados contidos em Ghazaryan et al. (2011) e suas referências.

Seja $L: D(L) \subset X \rightarrow X$ o operador linear com domínio

$$
D(L)=\left\{(u, w) \in X:\left(u^{\prime}, w^{\prime}\right) \in X \text { e }\left(u^{\prime \prime}, 0\right) \in X\right\}
$$

definido pela equação linear (1.27) dado por

$$
L\left(\begin{array}{l}
u \\
w
\end{array}\right)(\xi)=\left(\begin{array}{l}
u^{\prime \prime}(\xi)-c^{*} u^{\prime}(\xi)+f^{\prime}(\phi(\xi)) u(\xi)-w(\xi) \\
-c^{*} w^{\prime}(\xi)+\varepsilon(u(\xi)-\gamma w(\xi))
\end{array}\right)
$$

e indiquemos por $\sigma(L)$ o espectro de $L$.

O resultado básico que usaremos para obter estabilidade orbital foi utilizado por Yanagida (1989) e demonstrado por Bates e Jones (1989) e também por Ghazaryan et al. (2011) e consiste do seguinte

Teorema 1.2.2 A solução onda viajante $(\phi, \psi)$ da equação (1.23) é exponencialmente estável se

(i) Existe $\delta>0$ tal que $\sigma(L)$ satisfaz $\sigma(L) \backslash\{0\} \subset\{\mu \in \mathbb{C}: \operatorname{Re} \mu \leq-\delta\}$;

(ii) 0 é um autovalor simples de L, isto é, a equação

$$
\begin{aligned}
u^{\prime \prime}(\xi)-c^{*} u^{\prime}(\xi)+f^{\prime}(\phi(\xi)) u(\xi)-w(\xi) & =\phi^{\prime}(\xi) & \\
-c^{*} w^{\prime}(\xi)+\varepsilon(u(\xi)-\gamma w(\xi) & =\psi^{\prime}(\xi) & -\infty<x<\infty
\end{aligned}
$$

não tem solução limitada.

Para estudar o espectro de $L$, vamos recordar a seguinte

Definição 1.2.2 Sejam $L$ um operador linear e $\sigma(L)$ o espectro de $L$.

i) O espectro normal $\sigma_{n}(L)$ de $L$ é o conjunto de todos $\mu \in \mathbb{C}$ tais que $\mu$ é um autovalor isolado de multiplicidade finita.

ii) O espectro essencial $\sigma_{e}(L)$ é definido por $\sigma_{e}(L)=\sigma(L) \backslash \sigma_{n}(L)$.

Como $\phi(\xi) \rightarrow 0$ e $\phi(\xi) \rightarrow u_{2}$ quando $\xi \rightarrow \mp \infty$, os coeficientes do operador $L$ dado em (1.28) são assintoticamente constantes; tem, portanto, interesse estudar os seguintes operadores com coeficientes constantes $L_{-}$e $L_{+}$, com mesmos domínios que o de $L$, definidos por

$$
L_{-}\left(\begin{array}{l}
u \\
w
\end{array}\right)(\xi)=\left(\begin{array}{l}
u^{\prime \prime}(\xi)-c^{*} u^{\prime}(\xi)+f^{\prime}(0) u(\xi)-w(\xi) \\
-c^{*} w^{\prime}(\xi)+\varepsilon(u(\xi)-\gamma w(\xi))
\end{array}\right)
$$




$$
L_{+}\left(\begin{array}{l}
u \\
w
\end{array}\right)(\xi)=\left(\begin{array}{l}
u^{\prime \prime}(\xi)-c^{*} u^{\prime}(\xi)+f^{\prime}\left(u_{2}\right) u(\xi)-w(\xi) \\
-c^{*} w^{\prime}(\xi)+\varepsilon(u(\xi)-\gamma w(\xi))
\end{array}\right) .
$$

O fato fundamental aqui é que as diferenças $L-L_{-}$e $L-L_{+}$são da forma

$$
\left(L-L_{ \pm}\right) u(\xi)=a_{ \pm}(\xi) u(\xi),
$$

onde $a_{-}(\xi)=f^{\prime}(\phi(\xi))-f^{\prime}(0)$ e $a_{+}(\xi)=f^{\prime}(\phi(\xi))-f^{\prime}\left(u_{2}\right)$ e definem operadores compactos em $L^{2}\left(\mathbb{R}_{-}\right)$e em $L^{2}\left(\mathbb{R}_{+}\right)$, respectivamente, (ver Bates e Jones (1989) e Pego (1985)) e vale o seguinte resultado

Lema 1.2.1 Se $K: X \rightarrow X$ é um operador compacto, então $\sigma_{e}(L)=\sigma_{e}(L+K)$.

A demonstração do Lema 1.2.1 pode ser encontrada, por exemplo, em Henry (1981).

Dess a forma, o espectro essencial de $L$ pode ser obtido como a reunião dos espectros essenciais de $L_{-}$e de $L_{+}$, os quais estudaremos a seguir. Por comodidade, no que segue, indicaremos por $\alpha$ qualquer um dos números $f^{\prime}(0)$ ou $f^{\prime}\left(u_{2}\right)$. O fato essencial é que $\alpha$ é um número negativo: $\alpha<0$.

Vamos inicialmente analisar o resolvente de $L_{+}$. Um número $\lambda \in \mathbb{C}$ pertence ao conjunto resolvente de $L_{+}$se dado $(f, g) \in X$, existe um único $(u, w) \in D\left(L_{+}\right)$tal que

$$
\left(L_{+}-\lambda I\right)\left(\begin{array}{c}
u \\
w
\end{array}\right)=\left(\begin{array}{c}
f \\
g
\end{array}\right)
$$

e a aplicação $(f, g) \mapsto(u, w): X \rightarrow X$ é contínua.

A equação (1.32) pode ser escrita de forma equivalente como o sistema

$$
\frac{d}{d \xi} z(\xi)=M(\lambda) z(\xi)+h(\xi)
$$

onde

$$
M(\lambda)=\left[\begin{array}{ccc}
0 & 1 & 0 \\
\lambda-\alpha & c^{*} & 1 \\
\frac{\varepsilon}{c^{*}} & 0 & -\frac{\varepsilon \gamma+\lambda}{c^{*}}
\end{array}\right] \text { e } h(\xi)=\left(\begin{array}{c}
0 \\
f(\xi) \\
-g(\xi) / c^{*}
\end{array}\right)
$$

Lema 1.2.2 Uma condição necessária e suficiente para que (1.33) tenha solução limitada em $(-\infty, \infty)$ para toda h limitada é que $M(\lambda)$ não tenha autovalor no eixo imaginário. Além disso, se $M(\lambda)$ não tem autovalor no eixo imaginário, então para toda $h \in B C(\mathbb{R})$, existe uma única $z=K(h) \in B C(\mathbb{R})$ tal que $K(h)$ é a única solução limitada de $(1.33)$ e o operador $K: B C(\mathbb{R}) \rightarrow$ $B C(\mathbb{R})$ é linear e contínuo.

Aqui, $B C(\mathbb{R})$ indica o espaço das funções contínuas e limitadas com a norma do sup.

A demonstração pode ser encontrada em Hale (1980).

Portanto, o espectro essencial de $L_{+}$consiste dos números complexos $\lambda$ tais que $M(\lambda)$ tem algum autovalor no eixo imaginário. Seja $p(\mu)=\operatorname{det}(M(\lambda)-\mu I)$ polinômio característico de $M(\lambda)$. Então,

$$
p(\mu)=\operatorname{det}(M(\lambda)-\mu I)=\frac{1}{c^{*}}\left(\varepsilon \gamma+\lambda+c^{*} \mu\right)\left[\mu^{2}-c^{*} \mu+\alpha-\lambda\right]-\frac{\varepsilon}{c^{*}},
$$

e o espectro essencial de $L_{+}$é

$$
\sigma_{e}\left(L_{+}\right)=\{\lambda \in \mathbb{C}: \text { existe } \tau \in \mathbb{R} \text { tal que } p(i \tau)=0\} .
$$


Agora, a equação $p(i \tau)=0$ é equivalente a

$$
\left(\varepsilon \gamma+\lambda+i c^{*} \tau\right)\left[-\tau^{2}-i c^{*} \tau+\alpha-\lambda\right]-\varepsilon=0 .
$$

Escrevendo $\lambda=x+i y, \operatorname{com} x, y \in \mathbb{R}$, a equação acima se escreve como

$$
\left[(\varepsilon \gamma+x)+i\left(y+c^{*} \tau\right)\right]\left[\left(\alpha-\tau^{2}-x\right)-i\left(y+c^{*} \tau\right)\right]=\varepsilon .
$$

Efetuando o produto indicado e igualando partes real e imaginária, concluímos que $x$ e $y$ são soluções reais do sistema

$$
\left\{\begin{array}{l}
(\varepsilon \gamma+x)\left(\alpha-\tau^{2}-x\right)+\left(y+c^{*} \tau\right)^{2}=\varepsilon \\
\left(y+c^{*} \tau\right)\left[\alpha-\tau^{2}-x-\varepsilon \gamma-x\right]=0
\end{array}\right.
$$

A segunda equação implica que $y=-c^{*} \tau$ ou $x=\frac{1}{2}\left[\alpha-\varepsilon \gamma-\tau^{2}\right]$.

Se $y=-c^{*} \tau$, a primeira equação implica que $(\varepsilon \gamma+x)\left(\alpha-\tau^{2}-x\right)=\varepsilon$, isto é,

$$
x^{2}-\left(\alpha-\varepsilon \gamma-\tau^{2}\right) x+\varepsilon\left[1-\gamma\left(\alpha-\tau^{2}\right)=0 .\right.
$$

Segue-se então que

$$
x=x_{ \pm}(\tau)=\frac{1}{2}\left[\left(\alpha-\varepsilon \gamma-\tau^{2}\right) \pm \sqrt{\left(\alpha+\varepsilon \gamma-\tau^{2}\right)^{2}-4 \varepsilon}\right] .
$$

(para $\varepsilon>0$ suficientemente pequeno, o termo em $\sqrt{ }$ é positivo).

Substituindo $\tau=-y / c^{*}$, obtemos duas curvas no plano complexo que contém o espectro essencial de $L_{+}$; a curva mais à direita $x=x_{+}\left(-y / c^{*}\right)$ é dada por

$$
x_{+}=\frac{1}{2 c^{* 2}}\left[c^{* 2}(\alpha-\varepsilon \gamma)-y^{2}+\sqrt{c^{* 2}\left(\alpha+\varepsilon \gamma-y^{2}\right)^{2}-4 \varepsilon c^{* 2}}\right]
$$

que satisfaz

$$
\lim _{|y| \rightarrow \infty} x_{+}=\lim _{|\tau| \rightarrow \infty} x_{+}(\tau)=-\frac{1}{2} \lim _{|\tau| \rightarrow \infty} \frac{\left(\varepsilon \gamma+\alpha-\tau^{2}\right)^{2}-4 \varepsilon-\left(\varepsilon \gamma-\alpha+\tau^{2}\right)^{2}}{\left(\alpha-\varepsilon \gamma-\tau^{2}\right)+\sqrt{\left(\alpha+\varepsilon \gamma-\tau^{2}\right)^{2}-4 \varepsilon}}=-\varepsilon \gamma
$$

e tem o comportamento indicado na Figura 1.5.

Quando $x=\frac{1}{2}\left[\alpha-\varepsilon \gamma-\tau^{2}\right]$, os pontos $\lambda \in \sigma_{e}\left(L_{+}\right)$satisfazem $\operatorname{Re} \lambda \leq \frac{1}{2}[\alpha-\varepsilon \gamma]<-\varepsilon \gamma$, se $\varepsilon$ é suficientemente pequeno (por exemplo, $0<\varepsilon<\frac{1}{\gamma} \min \left\{-f^{\prime}(0),-f^{\prime}\left(u_{2}\right)\right\}$ ).

Procedendo da mesma forma, obtemos uma estimativa análoga para o espectro essencial de $L_{-}$. Demonstramos, portanto, o seguinte

Teorema 1.2.3 Se $\varepsilon>0$ é suficientemente pequeno, então existe $\delta \in(0, \varepsilon \gamma)$ tal que Re $\lambda<-\delta$, para todo $\lambda \in \sigma_{e}(L)$.

Uma vez que o espectro essencial de $L$ satisfaz a hipótese i) do Teorema 1.2.2, vamos analisar os autovalores de $L$ no semiplano $\operatorname{Re} \lambda \geq-\delta$. Para isso, queremos determinar $\lambda \in \mathbb{C} \operatorname{com} \operatorname{Re} \lambda \geq-\delta$ tal que a equação $L(u, w)=\lambda(u, w)$ tem solução não nula. Isso é equivalente a determinar $\lambda$ para os quais

$$
\left\{\begin{array}{l}
u^{\prime \prime}(\xi)-c^{*} u^{\prime}(\xi)+f^{\prime}(\phi(\xi)) u(\xi)-w(\xi)=\lambda u(\xi) \\
-c^{*} w^{\prime}(\xi)+\varepsilon(u(\xi)-\gamma w(\xi))=\lambda w(\xi)
\end{array}\right.
$$




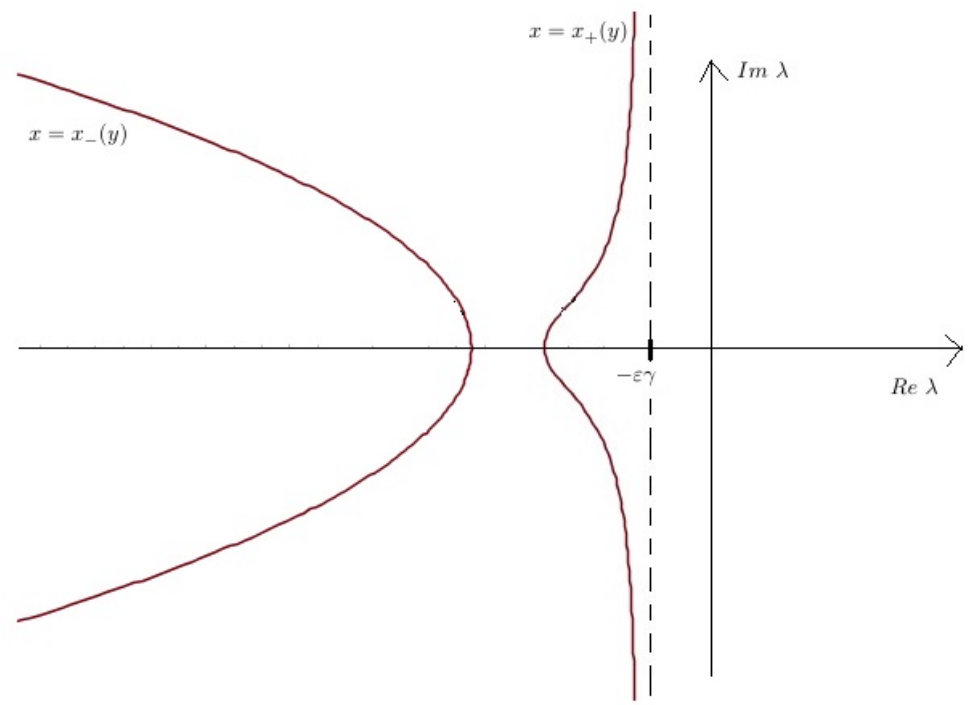

Figura 1.5: Comportamento de $x_{+}(y)$ e $x_{-}(y)$.

tem solução limitada não-nula.

Podemos escrever (1.35) na forma

$$
\frac{d}{d \xi} z(\xi)=M(\lambda, \xi) z(\xi)
$$

onde

$$
M(\lambda, \varepsilon)=\left[\begin{array}{ccc}
0 & 1 & 0 \\
\lambda-f^{\prime}(\phi) & c^{*} & 1 \\
\frac{\varepsilon}{c^{*}} & 0 & -\frac{\varepsilon \gamma+\lambda}{c^{*}}
\end{array}\right]
$$

ou ainda nas equações equivalentes:

$$
\frac{d}{d \xi} z(\xi)=\left(M_{1}(\lambda)+R_{1}(\xi)\right) z(\xi)
$$

onde

$$
M_{1}(\lambda)=\left[\begin{array}{ccc}
0 & 1 & 0 \\
\lambda-f^{\prime}(0) & c^{*} & 1 \\
\frac{\varepsilon}{c^{*}} & 0 & -\frac{\lambda+\varepsilon \gamma}{c^{*}}
\end{array}\right] \quad \text { e } \quad R_{1}(\xi)=\left[\begin{array}{ccc}
0 & 0 & 0 \\
f^{\prime}(0)-f^{\prime}(\phi(\xi)) & 0 & 0 \\
0 & 0 & 0
\end{array}\right]
$$

$$
\frac{d}{d \xi} z(\xi)=\left[M_{2}(\lambda)+R_{2}(\xi)\right] z(\xi)
$$

onde

$$
M_{2}(\lambda)=\left[\begin{array}{ccc}
0 & 1 & 0 \\
\lambda-f^{\prime}\left(u_{2}\right) & c^{*} & 1 \\
\frac{\varepsilon}{c^{*}} & 0 & -\frac{\lambda+\varepsilon \gamma}{c^{*}}
\end{array}\right] \quad \text { e } \quad R_{2}(\xi)=\left[\begin{array}{ccc}
0 & 0 & 0 \\
f^{\prime}\left(u_{2}\right)-f^{\prime}(\phi(\xi)) & 0 & 0 \\
0 & 0 & 0
\end{array}\right] .
$$

Lema 1.2.3 Suponha que $\alpha$ é um número real negativo. Se $\varepsilon>0$ é suficientemente pequeno, então 
existe uma constante $\delta_{1}>0$ tal que se Re $\lambda \geq-\delta_{1}$, então a matriz

$$
M(\lambda)=\left[\begin{array}{ccc}
0 & 1 & 0 \\
\lambda-\alpha & c^{*} & 1 \\
\frac{\varepsilon}{c^{*}} & 0 & -\frac{\lambda+\varepsilon \gamma}{c^{*}}
\end{array}\right]
$$

tem um autovalor com parte real positiva e dois autovalores com parte real negativa.

Demonstração. Como vimos na demonstração do Teorema 1.2.3, para todo $\alpha<0$, existe $\delta>0$ tal que se $M(\lambda)$ tem um autovalor no eixo imaginário, então $\operatorname{Re} \lambda<-\delta$. Portanto, tomando $0<\delta_{1} \leq \delta$, concluímos que se $\operatorname{Re} \lambda \geq-\delta_{1}$, então $M(\lambda)$ não tem autovalores no eixo imaginário. Logo, para $\operatorname{Re} \lambda \geq-\delta_{1}$, o número de autovalores de $M(\lambda)$ em cada um dos semiplanos $\operatorname{Re} \mu>0$ e $\operatorname{Re} \mu<0$ é constante.

Para determinar esse número, vamos calculá-lo quando $\lambda=0$. Os autovalores de $M(0)$ são as raízes do polinômio (1.34) $\operatorname{com} \lambda=0$, e se reduz a

$$
\left(\varepsilon \gamma+c^{*} \mu\right)\left[\mu^{2}-c^{*} \mu+\alpha\right]-\varepsilon=0 .
$$

Pelo Teorema da Função Implícita, essa última equação tem três raízes reais distintas dadas por

$$
\begin{gathered}
\mu_{3}(\varepsilon)=\mu_{-}-\frac{\varepsilon}{c^{*} \mu_{+} \sqrt{c^{* 2}-4 \alpha}}+O\left(\varepsilon^{2}\right), \\
\mu_{2}(\varepsilon)=\frac{1-\alpha \gamma}{c^{*} \alpha} \varepsilon+\frac{2(1-\alpha \gamma)}{c^{*} \alpha^{3}} \varepsilon^{2}+O\left(\varepsilon^{3}\right)
\end{gathered}
$$

e

$$
\mu_{1}(\varepsilon)=\mu_{+}+\frac{\varepsilon}{c^{*} \mu_{+} \sqrt{c^{* 2}-4 \alpha}}+O\left(\varepsilon^{2}\right),
$$

onde $\mu_{-}<0$ e $\mu_{+}>0$ são as raízes de $\mu^{2}-c^{*} \mu+\alpha=0$.

Como $\alpha<0$, temos $\mu_{3}(\varepsilon)<\mu_{2}(\varepsilon)<0<\mu_{1}(\varepsilon)$, para todo $\varepsilon>0$ suficientemente pequeno. Portanto, $M(\lambda)$ tem dois autovalores com parte real negativa e um autovalor com parte real positiva.

A seguir, vamos enunciar um resultado que será útil nas próximas seções

Teorema 1.2.4 Seja $A \in \mathbb{C}^{N \times N}$ uma matriz constante e $R$ uma matriz integrável satisfazendo

$$
\int_{0}^{\infty}\|R(t)\| d t<\infty
$$

Suponha que A é diagonalizável. Se $\lambda$ é um autovalor de $A$ e p é um autovetor correspondente, então o sistema

$$
\dot{x}=A x+R(t) x
$$

tem uma solução $\phi$ que satisfaz

$$
\lim _{t \rightarrow \infty} \phi(t) e^{-\lambda t}=p
$$

Demonstração. A demonstração pode ser encontrada no livro de Coddington e Levinson (1955), p. 104. 


\subsubsection{Função de Evans para sistema reduzido}

Considere o problema de autovalor

$$
u^{\prime \prime}-\bar{c} u^{\prime}+f^{\prime}(\bar{\phi}(\xi)) u=\lambda u, \quad-\infty<\xi<\infty .
$$

O problema (1.40) pode ser reescrito na equação

$$
\frac{d}{d \xi} Z_{0}(\xi, \lambda)=B(\xi, \lambda) Z_{0}(\xi, \lambda),
$$

onde $Z_{0}(\xi, \lambda)=(u, v)$ e

$$
B(\xi, \lambda)=\left(\begin{array}{cc}
0 & 1 \\
\lambda-f^{\prime}(\bar{\phi}(\xi)) & \bar{c}
\end{array}\right) .
$$

Como $\bar{\phi}(-\infty)=0$ e $\bar{\phi}(+\infty)=1$ podemos considerar

$$
\frac{d}{d \xi} Z_{0}(\xi, \lambda)=B_{1}(\lambda) Z_{0}(\xi, \lambda)
$$

onde

$$
B_{1}(\lambda)=\left(\begin{array}{cc}
0 & 1 \\
\lambda-f^{\prime}(0) & \bar{c}
\end{array}\right) \quad \text { quando } \quad \xi \rightarrow-\infty
$$

$$
\frac{d}{d \xi} Z_{0}(\xi, \lambda)=B_{2}(\lambda) Z_{0}(\xi, \lambda)
$$

onde

$$
B_{2}(\lambda)=\left(\begin{array}{cc}
0 & 1 \\
\lambda-f^{\prime}(1) & \bar{c}
\end{array}\right) \quad \text { quando } \quad \xi \rightarrow+\infty .
$$

Pelo Teorema A2 do Capítulo 5 de Henry (1981) sabemos que o espectro essencial está na região $\operatorname{Re} \lambda<-\delta_{0}<0$, onde $\delta_{0}=\min \left\{-f^{\prime}(0),-f^{\prime}(1)\right\}$.

Se $\operatorname{Re} \lambda \geq-\delta_{0}$, as matrizes (1.44) e (1.46) possuem autovalores satisfazendo, respectivamente:

$$
r_{-}<0<r_{+} \quad \text { e } \quad s_{-}<0<s_{+},
$$

e seus correspondentes autovetores:

$$
\rho_{ \pm}=\left(1, r_{ \pm}\right) \text {e } \quad \kappa_{ \pm}=\left(1, s_{ \pm}\right)
$$

Pelo Teorema 1.2.4, existe uma solução de (1.43) $Z_{0}^{+}$tal que

$$
\lim _{\xi \rightarrow-\infty} Z_{0}^{+}(\xi, \lambda) e^{-r_{+} \xi}=\rho_{+} .
$$

A menos de multiplicação por constantes, $Z_{0}^{+}$é a única solução limitada de (1.41) quando $\xi \rightarrow-\infty$.

Por outro lado (1.41) pode ser escrita na forma $Z_{0}^{\prime}=\left(B_{2}(\lambda)+R_{2}(\xi)\right) Z_{0}$, onde $B_{2}(\lambda)$ é dada por (1.46) e

$$
R_{2}(\lambda)=\left(\begin{array}{cc}
0 & 0 \\
f^{\prime}(1)-f^{\prime}(\bar{\phi}) & 0
\end{array}\right) .
$$

Portanto podemos definir uma solução $Z_{0}^{-}(\lambda, \xi)$ de (1.41) com a seguinte forma:

$$
Z_{0}^{-}(\lambda, \xi)=E_{0}(\lambda) \kappa_{+} e^{s_{+} \xi}+E_{1}(\lambda) \kappa_{-} e^{s_{-} \xi}+O\left(e^{s_{-} \xi}\right),
$$


onde $E_{i}, i=0,1$ são constantes em relação a $\xi$, mas dependem de $\lambda$.

A solução $Z_{0}^{-}$será limitada se, e somente se, $E_{0}(\lambda)=0$. Desta forma, podemos construir uma solução $Z_{0}$ tal que

$$
Z_{0} \rightarrow \rho_{+} e^{r_{+} \xi} \quad \text { quando } x \rightarrow-\infty
$$

e

$$
Z_{0} \rightarrow E_{0}(\lambda) \kappa_{+} e^{s_{+} \xi} \quad \text { quando } x \rightarrow \infty .
$$

Encontrar os valores de $\lambda$ tal que $E_{0}(\lambda)=0$ significa encontrar os valores de $\lambda$ para o qual $Z_{0}$ é uma solução limitada que é equivalente a encontrar os autovalores do problema (1.40). A função de Evans na literatura é apresentada de várias formas, esta última foi sugerida por Yanagida (1989). Outras formas da função de Evans podem ser encontradas em Kapitula e Promislow (2013), Jones (1984), Sandstede (2002) e referências.

Lema 1.2.4 Propriedades de $E_{0}(\lambda)$ :

(i) $E_{0}(\lambda)$ é analítica e real se $\lambda$ for real

(ii) $E_{0}(0)=0$

(iii) $\frac{d}{d \lambda} E_{0}(0) \neq 0$

Demonstração. Veja Yanagida (1985) Lema 4.7.

\subsection{Função de Evans para sistema FitzHugh-Nagumo}

Voltando ao problema (1.35), considerando $\alpha=f^{\prime}(0)$ ou $\alpha=f^{\prime}\left(u_{2}\right)$ em (1.37) ou em (1.38), sejam $r_{i}$ e $s_{i}, i=1,2,3$ os autovalores de $M_{1}(\lambda)$ e $M_{2}(\lambda)$, respectivamente, que satisfazem:

$$
\text { Re } r_{1}>0>\operatorname{Re} r_{2}>\operatorname{Re} r_{3} \text { e } \operatorname{Re} s_{1}>0>\operatorname{Re} s_{2}>\operatorname{Re} s_{3} \text {. }
$$

Sejam $\rho_{i}$ e $\kappa_{i}$ os autovetores correspondentes aos autovalores $r_{i}, s_{i}, i=1,2,3$, respectivamente, dados por

$$
\rho_{i}(\lambda, \varepsilon)=\left(1, r_{i}, \frac{\varepsilon}{c^{*} r_{i}+\lambda+\varepsilon \gamma}\right)
$$

$\mathrm{e}$

$$
\kappa_{i}(\lambda, \varepsilon)=\left(1, s_{i}, \frac{\varepsilon}{c^{*} s_{i}+\lambda+\varepsilon \gamma}\right) .
$$

Como $R_{2}(\xi)$ satisfaz as hipóteses do Teorema 1.2.4, existe uma base $\left\{\phi_{s_{1}}, \phi_{s_{2}}, \phi_{s_{3}}\right\}$ de soluções de (1.38) tal que $\phi_{s_{i}}(\xi) e^{-s_{i} \xi} \rightarrow \kappa_{i}$ quando $\xi \rightarrow+\infty$.

Da mesma forma, $R_{1}(\xi)$ também satisfaz as hipóteses do Teorema 1.2.4 e uma solução $\phi$ de (1.37) é limitada quando $\xi \rightarrow-\infty$ se, e somente se,

$$
\lim _{\xi \rightarrow-\infty} \phi(\xi) e^{-r_{1} \xi}=\rho_{1} .
$$

Escrevendo $\phi$ como combinação linear das funções da base $\left\{\phi_{s_{1}}, \phi_{s_{2}}, \phi_{s_{3}}\right\}$, isto é,

$$
\phi=c_{1} \phi_{s_{1}}+c_{2} \phi_{s_{2}}+c_{3} \phi_{s_{3}}
$$


concluímos que $\phi$ é limitada em $(-\infty, \infty)$ se, e somente se, a componente de $\phi$ na direção $\phi_{s_{1}}$ é zero, isto é, $c_{1}=0$. Da mesma forma como fizemos para o sistema reduzido, podemos construir uma solução $Z$ de (1.33) que satisfaz

$$
Z \rightarrow \rho_{1}(\lambda, \varepsilon) e^{r_{1}(\lambda, \varepsilon) \xi} \quad \text { quando } \quad \xi \rightarrow-\infty
$$

$$
Z \rightarrow E(\lambda, \varepsilon) \kappa_{1}(\lambda, \varepsilon) e^{s_{1}(\lambda, \varepsilon) \xi} \quad \text { quando } \xi \rightarrow \infty .
$$

Observemos que $M_{i}(\lambda), i=1,2$, tem autovalores distintos e dependem analiticamente de $\lambda$ para $\operatorname{Re} \lambda>-\delta$, concluímos que as soluções de (1.37) (ou equivalentemente, (1.38)) são funções analíticas de $\lambda$, bem como os autovalores e autovetores de $M_{i}(\lambda)$. Assim, a componente $c_{1}=c_{1}(\lambda)$ de $\phi$ também é uma função analítica de $\lambda$, definida no semiplano $\operatorname{Re} \lambda>-\delta$.

Definição 1.3.1 Para $\operatorname{Re} \lambda>-\delta$, definimos $E(\lambda):=c_{1}(\lambda)$, onde $c_{1}=c_{1}(\lambda)$ é a função definida no parágrafo anterior e dizemos que $E$ é a função de Evans associada ao problema (1.35).

O seguinte resultado é imediato

Teorema 1.3.1 Um número complexo $\lambda$ é autovalor de (1.35) se, e somente se, $E(\lambda)=0$.

Na referência Evans (1975), descreve as propriedades fundamentais da função $E$. No teorema a seguir, enunciamos as mais importantes, que estão ligadas ao tema de nosso estudo.

Teorema 1.3.2 Seja $\Omega \subset \mathbb{C}$ a componente do resolvente de $L$ que contém 0 . Então, a função $E$ pode ser estendida de modo analítico $a \Omega \times(0, \varepsilon)$ e tem as seguintes propriedades:

(i) E é analítica em $\lambda$ e é real se $\lambda \in \Omega$ é real;

(ii) $E(0, \varepsilon)=0$ e $\frac{d}{d \lambda} E(0, \varepsilon) \neq 0$ para todo $\varepsilon \in\left(0, \varepsilon_{0}\right)$;

(iii) $E(\lambda, \varepsilon) \neq 0$ para todo $\lambda \in \Omega \backslash\{0\}$ e $\varepsilon \in\left(0, \varepsilon_{0}\right)$.

As demonstrações dessas propriedades podem ser encontradas no Lema 4 em Yanagida (1989).

Observação: Derivando a equação

$$
\begin{gathered}
\phi^{\prime \prime}-c^{*} \phi^{\prime}+f(\phi)-\psi=0 \\
-c^{*} \psi^{\prime}+\varepsilon(\phi-\gamma \psi)=0
\end{gathered}
$$

obtemos

$$
\begin{gathered}
\phi^{\prime \prime \prime}-c^{*} \phi^{\prime \prime}+f^{\prime}(\phi) \phi^{\prime}-\psi^{\prime}=0 \\
-c^{*} \psi^{\prime \prime}+\varepsilon\left(\phi^{\prime}-\gamma \psi^{\prime}\right)=0 .
\end{gathered}
$$

portanto $\left(\phi^{\prime}, \psi^{\prime}\right)$ é uma autofunção associada ao autovalor $\lambda=0$ do problema (1.32).

O próximo lema estabelece uma importante relação entre $E(\lambda, \varepsilon)$ e $E_{0}(\lambda)$.

Lema 1.3.1 Existem funçôes $H_{1}(\lambda, \varepsilon)$ e $H_{2}(\lambda, \varepsilon)$ suaves em $\varepsilon$ e analíticas em $\lambda$ tal que

$$
E(\lambda, \varepsilon)=H_{1}(\lambda, \varepsilon)\left\{E_{0}(\lambda)+H_{2}(\lambda, \varepsilon)\right\} .
$$

Além disso $H_{1}(\lambda, \varepsilon) \neq 0$ para todo $\lambda$ e $H_{2}(\lambda, \varepsilon) \rightarrow 0$ quando $\varepsilon \rightarrow 0^{+}$.

Demonstração. Como mencionado na introdução, soluções ondas viajantes é uma família uniparamétrica da forma $\{(\phi(\cdot+k), \psi(\cdot+k)), k \in \mathbb{R}\}$, ou seja, as translações no espaço de ondas viajantes são ainda soluções ondas viajantes do mesmo sistema. Por isso vamos normalizar as trajetórias de $\Gamma_{\varepsilon}=\left(\phi(\xi), \phi^{\prime}(\xi), \psi(\xi)\right)$ do problema $(5)-(6)$ e de $\Gamma_{1}=\left(\bar{\phi}(\xi), \bar{\phi}^{\prime}(\xi)\right)$, a trajetória do problema (1.1) por

$$
\phi(0)=\bar{\phi}(0)=a .
$$


Pelo Teorema 1.1.3, $\Gamma_{\varepsilon} \rightarrow \Gamma_{0}$ quando $\varepsilon \rightarrow 0^{+}$, então

$$
\left(\phi(\xi, \varepsilon), \phi^{\prime}(\xi, \varepsilon), \psi(\xi, \varepsilon)\right) \rightarrow\left(\bar{\phi}(\xi), \bar{\phi}^{\prime}(\xi), 0\right) \quad \text { quando } \quad \varepsilon \rightarrow 0^{+} \text {e } \xi \text { fixo. }
$$

Existe uma função monótona decrescente $\xi_{0}(\varepsilon)$ satisfazendo $\xi_{0}(\varepsilon) \rightarrow+\infty$ quando $\varepsilon \rightarrow 0^{+}$tal que a condição de convergência (1.54) seja uniforme em $\left(-\infty, \xi_{0}(\varepsilon)\right)$. Então a trajetória de $\Gamma_{\varepsilon}$ é suficientemente próxima da trajetória de $\Gamma_{1}$ para $\xi \in\left(-\infty, \xi_{0}(\varepsilon)\right)$ e suficientemente próxima de $\Gamma_{2}$ para $\left(\xi_{0}(\varepsilon),+\infty\right)$ como mostra a Figura 1.6.



Figura 1.6: Trajetória de $\Gamma_{\varepsilon}$.

Vamos considerar o comportamento de $Z=\left(\phi, \phi^{\prime}, \psi\right)$ para $\xi \in\left(-\infty, \xi_{0}(\varepsilon)\right)$.

Como $r_{1}(\lambda, \varepsilon)$ é o único autovalor com parte real positiva para $\varepsilon \geq 0$ e $Z$ é contínua em $\varepsilon=0$ então, se $\xi$ é fixo;

$$
Z(\xi, \lambda, 0)=\lim _{\varepsilon \rightarrow 0^{+}} Z(\xi, \lambda, \varepsilon)
$$

e satisfaz a equação

$$
\frac{d}{d \xi} Z(\xi, \lambda, 0)=M(\xi, \lambda, 0) Z(\xi, \lambda, 0)
$$

onde

$$
M(\xi, \lambda, 0)=\left(\begin{array}{ccc}
B(\xi, \lambda) & & 0 \\
0 & 0 & \frac{-\lambda}{c}
\end{array}\right)
$$

e $B(\xi, \lambda)$ é a matriz (1.42). Desta forma, $Z(\xi, \lambda, 0)$ e $\left(\bar{\phi}(\xi), \bar{\phi}^{\prime}(\xi), 0\right)$ satisfazem a mesma equação. Por outro lado, a condição (1.50) pode ser escrita na forma

$$
Z(\xi, \lambda, 0) \simeq \rho_{1}(\lambda, 0) e^{r_{1}(\lambda, 0) \xi} \quad \text { quando } \xi \rightarrow-\infty .
$$


Fazendo $\xi \rightarrow-\infty$ na equação $(1.55)$ obtemos

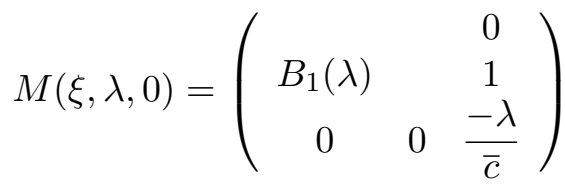

e isto significa que $r_{1}(\lambda, 0)=r_{+}(\lambda)$ e $\rho_{1}(\lambda, 0)=\left(\rho_{+}, 0\right)$. Como $Z(\xi, \lambda, 0)$ e $\left(Z_{0}(\xi, \lambda), 0\right)$ satisfazem a mesma condição quando $\xi \rightarrow-\infty$, isto implica que

$$
Z(\xi, \lambda, 0)=\left(\begin{array}{c}
Z_{0}(\xi, \lambda) \\
0
\end{array}\right), \quad-\infty<\xi<\infty .
$$

Podemos escolher $\xi_{0}(\varepsilon)$ de tal forma que a divergência de $\xi_{0}(\varepsilon)$ para o infinito é suficientemente lenta, obtemos

$$
Z\left(\xi_{0}(\varepsilon), \lambda, \varepsilon\right) \rightarrow\left(\begin{array}{c}
Z_{0}(\xi, \lambda) \\
0
\end{array}\right) \quad \operatorname{com} \varepsilon \rightarrow 0^{+} .
$$

Consequentemente, pela relação (1.49) obtemos

$$
Z\left(\xi_{0}(\varepsilon), \lambda, \varepsilon\right) \rightarrow E_{0}(\lambda)\left(\begin{array}{c}
\kappa_{+} \\
0
\end{array}\right) e^{\left[s_{+}(\lambda) \xi_{0}(\lambda)\right]}, \quad \varepsilon \rightarrow 0^{+}
$$

Vamos considerar o comportamento de $Z(\xi, \lambda, \varepsilon)$ para $\xi \in\left[\xi_{0}(\varepsilon),+\infty\right)$. Sejam

$$
\widetilde{M}(\lambda, \varepsilon, u)=\left(\begin{array}{ccc}
0 & 1 & 0 \\
\lambda-f^{\prime}(u) & c & 1 \\
\frac{\varepsilon}{c} & 0 & \frac{-(\lambda+\gamma \varepsilon)}{c}
\end{array}\right)
$$

e $\sigma_{i}(\lambda, \varepsilon, u), \quad i=1,2,3$, as raízes características de $\widetilde{M}$.

Como $f^{\prime}(u)<0$ para $u \in\left[u_{2}, 1\right]$, assumimos que

$$
\operatorname{Re}\left\{\sigma_{3}\right\}<\operatorname{Re}\left\{\sigma_{2}\right\}<0<\operatorname{Re}\left\{\sigma_{1}\right\}
$$

Sejam os autovetores

$$
v_{i}(\lambda, \varepsilon, u)=\left(1, \sigma_{i}, \frac{\varepsilon}{c \sigma_{i}+\lambda+\varepsilon \gamma}\right), \quad i=1,2,3 .
$$

Como os autoespaços dependem continuamente dos parâmetros podemos assumir, por uma normalização apropriada, que $v_{i}, \quad i=1,2,3$, depende continuamente de $\lambda, \varepsilon>0$ e $u \in\left[u_{2}, 1\right]$. Seja $V(\xi, \lambda, \varepsilon)$ uma matriz $3 \times 3$ função de $\xi$ cujas colunas são os vetores $v_{i}^{\prime} s$, isto é:

$$
V(\xi, \lambda, \varepsilon)=\left.\left(\begin{array}{lll}
v_{1} & v_{2} & v_{3}
\end{array}\right)\right|_{u=\phi(\xi, \varepsilon)} .
$$

Seja

$$
W(\xi, \lambda, \varepsilon) \equiv V^{-1}(\xi, \lambda, \varepsilon) Z(\xi, \lambda, \varepsilon) e^{I}
$$

onde

$$
I=-\int_{\xi_{0}(\varepsilon)}^{\xi} \sigma_{1}(\lambda, \varepsilon, \phi(s, \varepsilon)) d s-s_{+}(\lambda) \xi_{0}(\varepsilon) .
$$

Como




e $\phi\left(\xi_{0}(\varepsilon), \varepsilon\right) \rightarrow 1$ quando $\varepsilon \rightarrow 0^{+}, v_{1}\left(\lambda, \varepsilon, \phi\left(\xi_{0}(\varepsilon), \varepsilon\right)\right) \rightarrow\left(\kappa_{+}, 0\right)$. Portanto, pelas equações (1.57) e (1.58) obtemos

$$
W\left(\xi_{0}(\varepsilon), \lambda, \varepsilon\right) \simeq\left(\begin{array}{c}
E_{0}(\lambda) \\
0 \\
0
\end{array}\right) \quad \text { quando } \varepsilon \rightarrow 0^{+} .
$$

Por outro lado, de acordo com as equações (1.33) e (1.58)

$$
\begin{aligned}
\frac{d}{d \xi} W & =\frac{d}{d \xi}\left(V^{-1} Z\right) e^{I}-V^{-1} Z e^{I} \sigma_{1} \\
& =\left[\frac{d}{d \xi} V^{-1} Z+V^{-1} \frac{d}{d \xi} Z\right] e^{I}-V^{-1} Z e^{I} \sigma_{1} \\
& =\frac{d}{d \xi} V^{-1} Z e^{I}+V^{-1} M Z e^{I}-V^{-1} Z e^{I} \sigma_{1} \\
& =\frac{d}{d \xi} V^{-1} Z e^{I}+V^{-1} M V W-W \sigma_{1} \\
& =-V^{-2} \frac{d V}{d \xi} V W+N W,
\end{aligned}
$$

onde $N$ é uma matriz $3 \times 3$ tal que $N \equiv V^{-1} M V+\sigma_{1} I$. Logo $W$ satisfaz

$$
\frac{d}{d \xi} W=\left(N-V^{-1} \frac{d V}{d \xi}\right) W .
$$

Pela definição de $V$, a matriz $N$ tem a forma

$$
N(\xi, \lambda, \varepsilon)=\left(\begin{array}{ccc}
0 & 0 & 0 \\
0 & \sigma_{2}-\sigma_{1} & 0 \\
0 & 0 & \sigma_{3}-\sigma_{1}
\end{array}\right):=\left(\begin{array}{ccc}
0 & 0 & 0 \\
0 & \\
0 & N_{0}(\xi, \lambda, \varepsilon)
\end{array}\right) .
$$

Como

$$
\frac{d}{d \xi} \psi(\xi, \varepsilon)=\frac{\varepsilon}{c}(\phi(\xi, \varepsilon)-\gamma \psi(\xi, \varepsilon))>0
$$

podemos fazer a mudança de variáveis de $\xi$ para $\phi$ na equação (1.62). Usando (1.64) e (1.62) logo

$$
\begin{aligned}
& \frac{d W}{d \psi}=\frac{d W}{d \xi} \frac{d \xi}{d \psi} \\
& \quad=\left(N-V^{-1} \frac{d V}{d \xi}\right) W\left[\frac{-c}{\varepsilon\left(f^{-1}(\psi)-\gamma \psi\right)}\right] .
\end{aligned}
$$

Como

$$
\frac{d V}{d \psi}=\frac{d V}{d \xi}\left[\frac{-c}{\varepsilon\left(f^{-1}(\psi)-\gamma \psi\right)}\right]
$$

$\log 0$

$$
\frac{d W}{d \psi}=\left(\frac{-c}{\varepsilon\left(f^{-1}(\psi)-\gamma \psi\right)} N-V^{-1} \frac{d V}{d \psi}\right) W
$$

onde $f^{-1}$ é uma função inversa de $f$ a qual está definida para $\left[0, w_{2}\right]$ cuja imagem está sobre $\left[u_{2}, 1\right]$.

Por (1.63), a parte real das raízes características da matriz

$$
\frac{c}{\varepsilon\left(f^{-1}(\psi)-\varepsilon \psi\right)} N_{0}
$$

diverge para o infinito quando $\varepsilon \rightarrow 0^{+}$. Portanto, se resolvemos a equação (1.65) com a condição inicial (1.61) a segunda e a terceira componente de $W$ tende para zero quando $\varepsilon \rightarrow 0^{+}$para $\xi \geq \xi_{0}(\varepsilon)$. Logo o comportamento da primeira componente $W_{1}$ de $W$ é descrita aproximadamente 
pela equação

$$
\left.\frac{d}{d \psi} W_{1} \simeq\left(-V^{-1} \frac{d V}{d \psi}\right)\right|_{11} W_{1}, \quad \varepsilon \rightarrow 0^{+},
$$

onde $\left.(\cdot)\right|_{11}$ denota a componente $a_{11}$ da matriz. Como $V$ é suave em $\varepsilon=0$, então

$$
\left.\left(-V^{-1} \frac{d V}{d \psi}\right)\right|_{11}
$$

tem um limite finito quando $\varepsilon \rightarrow 0^{+}$.

Como a equação (1.66) é homogênea em $W_{1}$, logo $W_{1}$ pode ser escrita como

$$
W_{1}(+\infty, \lambda, \varepsilon) \simeq J(\lambda, \varepsilon) E_{0}(\lambda), \quad \varepsilon \rightarrow 0^{+},
$$

onde $J(\lambda, \varepsilon)$ é analítica em $\lambda \in \mathbb{C}$, suave em $\varepsilon \geq 0$, positiva para $\lambda$ real e $J(\lambda, \varepsilon) \neq 0$ para qualquer $\lambda$ e $\varepsilon \geq 0$.

Consequentemente,

$$
W(+\infty, \lambda, \varepsilon) \simeq\left[\begin{array}{c}
J(\lambda, \varepsilon) E_{0}(\lambda) \\
0 \\
0
\end{array}\right], \quad \varepsilon \rightarrow 0^{+} .
$$

Como $v_{1}\left(\lambda, \varepsilon, u_{2}\right)=\kappa_{1}(\lambda, \varepsilon)$ e $\sigma_{1}\left(\lambda, \varepsilon, u_{2}\right)=s_{1}(\lambda, \varepsilon)$ segue das equações (1.58) e (1.67) que

$$
Z(\xi, \lambda, \varepsilon) \simeq \kappa_{1}(\lambda, \varepsilon) H_{1}(\lambda, \varepsilon)\left[E_{0}(\lambda)+H_{2}(\lambda, \varepsilon)\right] e^{s_{1}(\lambda, \varepsilon) \xi}, \quad \xi \rightarrow+\infty,
$$

onde

$$
H_{1}(\lambda, \varepsilon) \equiv J(\lambda, \varepsilon) \exp \left[\int_{\xi_{0}(\varepsilon)}^{+\infty}\left(\sigma_{1}(\lambda, \varepsilon, \phi(\xi, \varepsilon))-s_{1}(\lambda, \varepsilon) d \xi+s_{+} \xi_{0}(\varepsilon)\right]\right.
$$

e

$$
H_{2}(\lambda, \varepsilon) \equiv \frac{E(\lambda, \varepsilon)}{H_{1}(\lambda, \varepsilon)}-E_{0}(\lambda) .
$$

Teorema 1.3.3 Se $\varepsilon>0$ for suficientemente pequeno, a onda viajante $(\phi(\xi), \psi(\xi))$ do problema (1) é exponencialmente estável, isto é, existem constantes $K>0$ e $\omega>0$ tais que para algum $x_{0} \in \mathbb{R}$

$$
\left|u(x, t)-\phi\left(x+c t-x_{0}\right)\right|<K e^{-\omega t}
$$

$e$

$$
\left|w(x, t)-\psi\left(x+c t-x_{0}\right)\right|<K e^{-\omega t}
$$

para todo $x \in \mathbb{R}$ e todo $t \geq 0$.

Demonstração. Seja $\delta_{1}>0$ a constante do Lema 1.2.3. Seja $R_{0}>0$ uma constante independente de $\varepsilon$ e considere

$$
\begin{aligned}
C_{1} & \equiv\left\{\lambda \in \mathbb{C} ;|\lambda| \leq R_{0}, \operatorname{Re}\{\lambda\} \geq-\delta_{1}\right\} \\
C_{2} & \equiv\left\{\lambda \in \mathbb{C} ;|\lambda|>R_{0}, \operatorname{Re}\{\lambda\} \geq-\delta_{1}\right\} .
\end{aligned}
$$

Sejam $R_{0}$ fixo e $\varepsilon>0$ suficientemente pequeno. Como $E_{0}(0)=0$, usando o Teorema 1.3 .2 e o Lema 1.3.1 temos

$$
H_{1}(0, \varepsilon) H_{2}(0, \varepsilon)=0 \quad \Longrightarrow \quad H_{2}(0, \varepsilon)=0 \text {. }
$$

Como $C_{1}$ é compacto, a convergência $H_{2}(\lambda, \varepsilon) \rightarrow 0$ quando $\varepsilon \rightarrow 0^{+}$é uniforme em $C_{1}$. Pelo fato que $E_{0}(\lambda) \neq 0$ em $C_{1} \backslash\{\lambda \in \mathbb{C} ; \operatorname{Re}\{\lambda\}<0\}$. Logo $\lambda=0$ é o único zero de $E_{0}(\lambda)+H_{2}(\lambda, \varepsilon)$ em $C_{1} \backslash\{\lambda \in \mathbb{C} ; \operatorname{Re}\{\lambda\}<0\}$. 
Agora vamos provar que não existe autovalor em $C_{2}$ se $R_{0}$ é suficientemente grande.

Se $\varepsilon>0$ é suficientemente pequeno e $\operatorname{Re}\{\lambda\}>-\delta_{1}$, existe $k>0$ tal que as raízes de $M_{1}(\lambda, \varepsilon)$ satisfazem:

$$
\begin{aligned}
& r_{1}(\lambda, \varepsilon)=\frac{c}{2}+\frac{\sqrt{c^{2}+4\left(\lambda-f^{\prime}(0)\right)}}{2}+k(\varepsilon), \\
& r_{2}(\lambda, \varepsilon)=\frac{c}{2}-\frac{\sqrt{c^{2}+4\left(\lambda-f^{\prime}(0)\right)}}{2}+k(\varepsilon), \\
& r_{3}(\lambda, \varepsilon)=-\frac{\lambda}{2}+k(\varepsilon) .
\end{aligned}
$$

Como

$$
\frac{r_{1}(\lambda, \varepsilon)}{\sqrt{\lambda}}=\frac{1}{2}\left\{\frac{c+k(\varepsilon)}{\sqrt{\lambda}}+\sqrt{4+\frac{c^{2}-f^{\prime}(0)}{\lambda}}\right\},
$$

$\log 0$

$$
\lim _{\lambda \rightarrow+\infty} \frac{r_{1}(\lambda, \varepsilon)}{\sqrt{\lambda}}=1 \quad \Longrightarrow \quad \lim _{\lambda \rightarrow+\infty} \frac{r_{1}(\lambda, \varepsilon)-\sqrt{\lambda}}{\sqrt{\lambda}}=0
$$

e podemos concluir que

$$
r_{1}(\lambda, \varepsilon)=\sqrt{\lambda}+O(1) \quad \text { quando } \quad \lambda \rightarrow+\infty
$$

De forma análoga, concluímos que

$$
\begin{aligned}
& r_{2}(\lambda, \varepsilon)=-\sqrt{\lambda}+O(1), \\
& r_{3}(\lambda, \varepsilon)=-\frac{\lambda}{c}+O(1), \quad \text { quando } \quad|\lambda| \rightarrow+\infty .
\end{aligned}
$$

O mesmo vale para as raízes do polinômio característico de $M_{2}$

$$
\begin{aligned}
& s_{1}(\lambda, \varepsilon)=\sqrt{\lambda}+O(1) \\
& s_{2}(\lambda, \varepsilon)=-\sqrt{\lambda}+O(1) \\
& s_{3}(\lambda, \varepsilon)=-\frac{\lambda}{c}+O(1) \quad \text { quando }|\lambda| \rightarrow+\infty .
\end{aligned}
$$

Sejam as matrizes $P(\lambda, \varepsilon)$ e $Q(\lambda, \varepsilon)$ cujas colunas são os autovetores associados aos autovalores $r_{i}^{\prime} s$ e $s_{i}^{\prime} s$, isto é,

$$
P(\lambda, \varepsilon)=\left(\begin{array}{ccc}
1 & 1 & 1 \\
r_{1} & r_{2} & r_{3} \\
\frac{\varepsilon}{c r_{1}+\lambda+\varepsilon \gamma} & \frac{\varepsilon}{c r_{2}+\lambda+\varepsilon \gamma} & \frac{\varepsilon}{c r_{3}+\lambda+\varepsilon \gamma}
\end{array}\right)
$$

e

$$
Q(\lambda, \varepsilon)=\left(\begin{array}{ccc}
1 & 1 & 1 \\
s_{1} & s_{2} & s_{3} \\
\frac{\varepsilon}{c s_{1}+\lambda+\varepsilon \gamma} & \frac{\varepsilon}{c s_{2}+\lambda+\varepsilon \gamma} & \frac{\varepsilon}{c s_{3}+\lambda+\varepsilon \gamma}
\end{array}\right)
$$

Considere

$$
\begin{aligned}
& X_{1}(\xi, \lambda, \varepsilon) \equiv P^{-1}(\lambda, \varepsilon) Z(\xi, \lambda, \varepsilon) \\
& X_{2}(\xi, \lambda, \varepsilon) \equiv Q^{-1}(\lambda, \varepsilon) Z(\xi, \lambda, \varepsilon)
\end{aligned}
$$


Por (1.36), (1.37) e (1.75), $X_{1}(\xi, \lambda, \varepsilon)$ satisfaz

$$
\begin{aligned}
\frac{d}{d \xi} X_{1}(\xi, \lambda, \varepsilon) & =P^{-1}(\lambda, \varepsilon) \frac{d}{d \xi} Z(\xi, \lambda, \varepsilon) \\
& =P^{-1}(\lambda, \varepsilon) M(\xi, \lambda, \varepsilon) Z(\xi, \lambda, \varepsilon) \\
& =P^{-1}(\lambda, \varepsilon)\left[M_{1}(\lambda, \varepsilon)+R_{1}(\xi)\right] Z(\xi, \lambda, \varepsilon) \\
& =P^{-1}(\lambda, \varepsilon) M_{1}(\lambda, \varepsilon) P(\lambda, \varepsilon) X_{1}(\xi, \lambda, \varepsilon)+P^{-1}(\lambda, \varepsilon) R_{1}(\xi) P(\lambda, \varepsilon) X_{1}(\xi, \lambda, \varepsilon) .
\end{aligned}
$$

Desta forma podemos escrever

$$
\frac{d}{d \xi} X_{1}(\xi, \lambda, \varepsilon)=\left[N_{1}(\lambda, \varepsilon)+\tilde{N}_{1}(\xi, \lambda, \varepsilon)\right] X_{1}(\xi, \lambda, \varepsilon)
$$

onde

$$
N_{1}=\left(\begin{array}{ccc}
r_{1} & 0 & 0 \\
0 & r_{2} & 0 \\
0 & 0 & r_{3}
\end{array}\right)
$$

e $\tilde{N}_{1}(\xi, \lambda, \varepsilon)=P^{-1}(\lambda, \varepsilon) R_{1}(\xi) P(\lambda, \varepsilon)$.

Usando (1.70), vemos que todas as componentes de $\tilde{N}_{1}$ tendem para zero quando $|\lambda| \rightarrow+\infty$, ou seja, quando $\xi$ é fixo e $|\lambda| \rightarrow+\infty$ todas as componentes de

$$
X_{1}(\xi, \lambda, \varepsilon)-\left(\begin{array}{l}
1 \\
0 \\
0
\end{array}\right) e^{r_{1}} \xi
$$

tornam-se pequenas em comparação com $e^{r_{1} \xi}$.

Logo, por (1.75),

$$
Z(\xi, \lambda, \varepsilon)=\rho_{1} e^{r_{1} \xi} \quad \Longrightarrow \quad Z(0, \lambda, \varepsilon)=\rho_{1} \quad \text { quando } \quad|\lambda| \rightarrow+\infty
$$

e pela igualdade (1.70) temos

$$
Z(0, \lambda, \varepsilon)=\left(\begin{array}{c}
1+o(1) \\
\sqrt{\lambda}++O(1) \\
o(1)
\end{array}\right) \quad \text { quando } \quad|\lambda| \rightarrow+\infty .
$$

Por outro lado, $X_{2}$ satisfaz

$$
\frac{d}{d \xi} X_{2}(\xi, \lambda, \varepsilon)=\left[N_{2}(\lambda, \varepsilon)+\tilde{N}_{2}(\xi, \lambda, \varepsilon)\right] X_{2}(\xi, \lambda, \varepsilon),
$$

onde

$$
N_{2}=\left(\begin{array}{ccc}
s_{1} & 0 & 0 \\
0 & s_{2} & 0 \\
0 & 0 & s_{3}
\end{array}\right)
$$

e $\tilde{N}_{2}(\xi, \lambda, \varepsilon)=Q^{-1}(\lambda, \varepsilon) R_{2}(\xi) Q(\lambda, \varepsilon)$. De maneira similar concluímos que

$$
Z(0, \lambda, \varepsilon)=E(\lambda, \varepsilon)\left(\begin{array}{c}
1+o(1) \\
\sqrt{\lambda}++O(1) \\
o(1)
\end{array}\right) \quad \text { quando } \quad|\lambda| \rightarrow+\infty .
$$

Comparando (1.77) com (1.76) obtemos $E(\lambda, \varepsilon) \rightarrow 1$ quando $|\lambda| \rightarrow+\infty$.

Como o comportamento das raízes características $r_{i}$ e $s_{i}, i=1,2,3$, quando $|\lambda| \rightarrow+\infty$, não depende essencialmente de $\varepsilon$, a convergência de $E(\lambda, \varepsilon)$ é uniforme para $\varepsilon$ pequeno. Pelo Teorema 1.3.1, existe uma constante $R_{0}>0$, independente de $\varepsilon$, tal que $C_{2}$ não contém autovalor da equação 
(1.35).

Vamos provar que $\lambda=0$ é um autovalor simples.

A equação (1.29) pode ser reescrita na forma

$$
\frac{d}{d \xi} \hat{Z}(\xi, \varepsilon)=M(\xi, 0, \varepsilon) \hat{Z}(\xi, \varepsilon)+\left(\begin{array}{c}
0 \\
\phi^{\prime}(\xi, \varepsilon) \\
-\frac{\psi^{\prime}(\xi, \varepsilon)}{c^{*}}
\end{array}\right) .
$$

Mostrar que 0 é um autovalor simples é mostrar que (1.78) não tem solução limitada.

Seja $Z(\xi, \lambda, \varepsilon)$ a única solução de (1.36), a menos de multiplicação por constantes, que é limitada quando $\xi \rightarrow-\infty$.

Como $\left(\phi^{\prime}, \psi^{\prime}\right)$ satisfaz (1.52) e é uma solução limitada de (1.36), podemos escrever

$$
\hat{Z}(\xi, 0, \varepsilon)=C_{1}\left(\begin{array}{c}
\phi^{\prime} \\
\phi^{\prime \prime} \\
\psi^{\prime}
\end{array}\right)
$$

para alguma constante $C_{1} \neq 0$. Substituindo $\hat{Z}(\xi, \lambda, \varepsilon)$ na equação (1.36) e diferenciando em $\lambda$, temos

$$
\begin{aligned}
\left.\frac{d}{d \lambda}\left(\frac{d}{d \xi} \hat{Z}(\xi, \lambda, \varepsilon)\right)\right|_{\lambda=0} & =\left.\frac{d}{d \lambda}(M(\xi, \lambda, \varepsilon) \hat{Z}(\xi, \lambda, \varepsilon))\right|_{\lambda=0} \\
\frac{d}{d \xi} \hat{Z}_{\lambda}(\xi, 0, \varepsilon) & =\left.\frac{d}{d \lambda} M(\xi, \lambda, \varepsilon)\right|_{\lambda=0} \hat{Z}(\xi, 0, \varepsilon)+\left.M(\xi, 0, \varepsilon) \frac{d}{d \lambda} \hat{Z}(\xi, \lambda, \varepsilon)\right|_{\lambda=0} \\
& =\left(\begin{array}{ccc}
0 & 0 & 0 \\
1 & 0 & 0 \\
0 & 0 & -\frac{1}{c^{*}}
\end{array}\right) C_{1}\left(\begin{array}{c}
\phi^{\prime} \\
\phi^{\prime \prime} \\
\psi^{\prime}
\end{array}\right)+M(\xi, 0, \varepsilon) \hat{Z}_{\lambda}(\xi, 0, \varepsilon),
\end{aligned}
$$

ou seja

$$
\frac{d}{d \xi} \hat{Z}_{\lambda}(\xi, 0, \varepsilon)=+M(\xi, 0, \varepsilon) \hat{Z}_{\lambda}(\xi, 0, \varepsilon)+C_{1}\left(\begin{array}{c}
0 \\
\phi^{\prime} \\
-\frac{\psi^{\prime}}{c^{*}}
\end{array}\right)
$$

isto significa que $\frac{1}{C_{1}} Z_{\lambda}(\xi, 0, \varepsilon)$ é uma solução particular da equação (1.78). Por outro lado, é a única solução, a menos de multiplicação de contantes, da equação (1.36) com $\lambda=0$ que é limitada quando $\xi \rightarrow-\infty$. Isto significa que qualquer solução da equação (1.78) que é limitada quando $\xi \rightarrow-\infty$ pode ser escrita como

$$
\hat{Z}(\xi, \varepsilon)=\left(\frac{1}{B_{1}}\right) \hat{Z}_{\lambda}(\xi, 0, \varepsilon)+C_{2}\left(\begin{array}{c}
\phi^{\prime}(\xi, \varepsilon) \\
\phi^{\prime \prime}(\xi, \varepsilon) \\
\psi^{\prime}(\xi, \varepsilon)
\end{array}\right)
$$

para alguma constante $C_{2}$.

Diferenciando a equação (1.51)

$$
\begin{aligned}
\frac{d}{d \lambda} \hat{Z}(\xi, 0, \varepsilon) & =\left.\frac{d}{d \lambda}\left(E(\lambda, \varepsilon) \kappa_{1}(\lambda, \varepsilon) e^{s_{1}(\lambda, \varepsilon) \xi}\right)\right|_{\lambda=0} \\
& =E_{\lambda}(0, \varepsilon) \kappa_{1}(0, \varepsilon) e^{s_{1}(0, \varepsilon) \xi}+\left.E(0, \varepsilon) \frac{d}{d \lambda} \kappa_{1}(\lambda, \varepsilon) e^{s_{1}(\lambda, \varepsilon) \xi}\right|_{\lambda=0}
\end{aligned}
$$

Como $E(0, \varepsilon)=0$ e $E_{\lambda}(0, \varepsilon) \neq 0$ então $\hat{Z}_{\lambda}$ não é limitada quando $\xi \rightarrow \infty$ e portanto a equação 
(1.78) não tem solução limitada e $\lambda=0$ é um autovalor simples. Pelo Teorema 1.2.2 segue que a onda viajante $(\phi, \psi)$ é exponencialmente estável.

\subsection{Outros resultados - estimativas uniformes}

Considere o problema de Cauchy

$$
\left\{\begin{array}{l}
y_{t}=y_{x x}-c y_{x}+f(y)-z \\
z_{t} \quad-c z_{x}+\varepsilon(y-\gamma z) \\
y(x, 0)=y_{0}(x) \quad \text { e } \quad z(x, 0)=z_{0}(x),
\end{array}\right.
$$

onde $0 \leq y_{0}(x) \leq u_{2}$ e $0 \leq z_{0}(x) \leq w_{2}$.

O resultado abaixo é um teorema da comparação para o problema (1.81) e foi enunciado em Klaasen e Troy (1981), Tuma e Blázquez (1992) e demonstrado para casos gerais em Tuma (1987).

Teorema 1.4.1 Considere

$$
\left\{\begin{array}{l}
N_{1}(y, z) \equiv y_{t}-y_{x x}+c y_{x}-f(y)+z=0 \\
N_{2}(y, z) \equiv z_{t}+c z_{x}-\varepsilon(y-\gamma z)=0 . \\
y(x, 0)=y_{0}(x) \quad z(x, 0)=z_{0}(x)
\end{array}\right.
$$

Sejam $y, z, \underline{y}, \underline{z}$ soluções limitadas de classe $C^{2} \operatorname{com} N_{1}(y, z) \geq 0, N_{2}(y, z) \leq 0, N_{1}(\underline{y}, \underline{z}) \leq 0 e$ $N_{2}(\underline{y}, \underline{z}) \geq 0$ em $\mathbb{R} \times \mathbb{R}^{+}$.

$\bar{S}$ e $\underline{y}(x, 0) \leq y(x, 0)$ e $z(x, 0) \leq \underline{z}(x, 0)$ para todo $x \in \mathbb{R}$, então $\underline{y}(x, t) \leq y(x, t)$ e $z(x, t) \leq \underline{z}(x, t)$ para todo $(x, t) \in \mathbb{R} \times \mathbb{R}^{+}$.

Lema 1.4.1 Seja $f$ satisfazendo as hipóteses (H1)-(H4). Suponha que

$$
\begin{array}{ll}
\lim _{x \rightarrow-\infty} \sup y_{0}(x)<u_{1} & \lim _{x \rightarrow \infty} \inf y_{0}(x)>u_{1} . \\
\lim _{x \rightarrow-\infty} \sup z_{0}(x)<w_{1} & \lim _{x \rightarrow \infty} \inf z_{0}(x)>w_{1} .
\end{array}
$$

Então, existem constantes $x_{1}, x_{2} \in \mathbb{R}$ e $k_{1}, k_{2}, \nu>0$ tais que

$$
\begin{aligned}
& \phi\left(x-x_{1}\right)-k_{1} e^{-\nu t} \leq y(x, t) \leq \phi\left(x-x_{2}\right)+k_{1} e^{-\nu t} \\
& \psi\left(x-x_{2}\right)-k_{2} e^{-\nu t} \leq z(x, t) \leq \psi\left(x-x_{1}\right)+k_{2} e^{-\nu t}
\end{aligned}
$$

para todo $(x, t) \in \mathbb{R} \times \mathbb{R}^{+}$

Demonstração. Vamos provar as desigualdades

$$
\begin{aligned}
\phi\left(x-x_{1}\right)-k_{1} e^{-\nu t} \leq & y(x, t) \\
& z(x, t) \leq \psi\left(x-x_{1}\right)+k_{2} e^{-\nu t} .
\end{aligned}
$$

Para isto vamos definir as seguintes funções

$$
\begin{aligned}
& \underline{y}(x, t)=\phi(x-n(t))-m_{1}(t) \\
& \underline{z}(x, t)=\psi(x-n(t))+m_{2}(t)
\end{aligned}
$$


escolhendo as funções $n(t), m_{1}(t)$ e $m_{2}(t)$ de tal forma que $N_{1}(\underline{y}, \underline{z}) \leq 0$ e $N_{2}(\underline{y}, \underline{z}) \geq 0$ em $\mathbb{R} \times \mathbb{R}^{+}$ e $\underline{y}(x, 0) \leq y(x, 0)$ e $z(x, 0) \leq \underline{z}(x, 0)$ para todo $x \in \mathbb{R}$. As desigualdades (1.85) serão consequências do Teorema 1.4.1.

Considere $m_{1}(t)=k_{1} e^{-\nu t}$ e $m_{2}(t)=k_{2} e^{-\nu t}$, onde as constantes $k_{1}$ e $k_{2}$ satisfazem

$$
u_{1}<u_{2}-k_{1}<\lim _{x \rightarrow \infty} \inf y_{0}(x) \quad \text { e } \quad w_{1}>k_{2}>\lim _{x \rightarrow \infty} \inf z_{0}(x)
$$

Seja $x^{*}$ suficientemente grande tal que

$$
\begin{aligned}
& \phi\left(x-x^{*}\right)-k_{1} \leq y_{0}(x)=y(x, 0) \\
& \psi\left(x-x^{*}\right)+k_{2} \geq z_{0}(x)=z(x, 0)
\end{aligned}
$$

onde $n(0)=x^{*}$.

Seja $\tau=x-n(t)$,

$$
\begin{aligned}
N_{1}(\underline{y}, \underline{z}) & =\underline{y}_{t}-\underline{y}_{x x}+c \underline{y}_{x}-f(\underline{y})+\underline{z} \\
& =-n^{\prime}(t) \phi^{\prime}(\tau)-m_{1}^{\prime}(t)-\phi^{\prime \prime}(\tau)+c \phi^{\prime}(\tau)-f\left(\phi-m_{1}\right)+\psi+m_{2} \\
& =-n^{\prime}(t) \phi^{\prime}(\tau)-m_{1}^{\prime}(t)+f(\phi)-f\left(\phi-m_{1}\right)+m_{2}(t),
\end{aligned}
$$

pois $\phi^{\prime \prime}(\tau)-c \phi^{\prime}(\tau)+f(\phi)-\psi=0$.

$$
\begin{aligned}
N_{2}(\underline{y}, \underline{z}) & =\underline{z}_{t}+c \underline{z}_{x}-\varepsilon(\underline{y}-d \underline{z}) \\
& =-n^{\prime}(t) \psi^{\prime}(\tau)+m_{2}^{\prime}(t)+c \psi^{\prime}(\tau)-\varepsilon\left(\phi-m_{1}-\gamma\left(\psi+m_{2}\right)\right) \\
& =-n^{\prime}(t) \psi^{\prime}(\tau)+m_{2}^{\prime}(t)+\varepsilon m_{1}(t)+\varepsilon \gamma m_{2}(t),
\end{aligned}
$$

pois $c \psi^{\prime}(\tau)-\varepsilon(\phi-\gamma \psi)=0$

Seja $\delta>0$, vamos considerar o intervalo $\delta \leq \phi \leq u_{2}-\delta$, nesta faixa sabemos que $\phi^{\prime}(\tau), \psi^{\prime}(\tau) \geq \beta$ para algum $\beta>0$.

Pela diferenciabilidade de $f$ temos, $f(\phi)-f\left(\phi-m_{1}\right) \leq C m_{1}$, para alguma constante $C>0$. Se $n^{\prime}(t)>0$ então

$$
N_{1}(\underline{y}, \underline{z}) \leq-n^{\prime}(t) \beta+(C+\nu) m_{1}+m_{2}
$$

Como $\phi^{\prime}( \pm \infty), \psi^{\prime}( \pm \infty)=0$, então existe $M>0$ tal que $\phi^{\prime}(\tau), \psi^{\prime}(\tau)<M$ para todo $\tau \in \mathbb{R}$ e

$$
N_{2}(\underline{y}, \underline{z}) \geq-n^{\prime}(t) M+\varepsilon m_{1}+(\varepsilon \gamma-\nu) m_{2} .
$$

Existem constantes positivas $\hat{\gamma}_{1}$ e $\hat{\gamma}_{2}$ tais que

$$
\begin{aligned}
& N_{1}(\underline{u}, \underline{w}) \leq-n^{\prime}(t) \beta+\hat{\gamma}_{1} m_{1}+\hat{\gamma}_{2} m_{2} \\
& N_{2}(\underline{u}, \underline{w}) \geq-n^{\prime}(t) M+\hat{\gamma}_{1} m_{1}+\hat{\gamma}_{2} m_{2} \quad \text { se } \quad n^{\prime}(t) \geq 0 .
\end{aligned}
$$

Basta tomar $\varepsilon \gamma>\nu, C+\nu \leq \hat{\gamma}_{1} \leq n$ e $1 \leq \hat{\gamma}_{1} \leq \varepsilon \gamma-\nu$. Como $\beta<\phi^{\prime}(\tau)<M$ então sejam

$$
n^{\prime}(t)=\frac{\hat{\gamma}_{1} m_{1}+\hat{\gamma}_{2} m_{2}}{\beta}
$$


e $n(0)=x^{*}$. Então $n(t)=x_{1}+\bar{x}_{1} e^{-\nu t}$, onde

$$
x_{1}=x^{*}+\frac{\hat{\gamma}_{1} k_{1}+\hat{\gamma}_{2} k_{2}}{\nu \beta} \quad \text { e } \quad \bar{x}_{1}=\frac{\hat{\gamma}_{1} k_{1}+\hat{\gamma}_{2} k_{2}}{-\nu \beta} .
$$

Note que $n^{\prime}(t) \geq 0$ e $n(t) \rightarrow x_{1}$ quando $t \rightarrow \infty$ e com essa escolha, $N_{1}(\underline{y}, \underline{z}) \leq 0$ e $N_{2}(\underline{y}, \underline{z}) \geq 0$ para $\delta<\phi<u_{2}-\delta$.

Agora considere

$$
J\left(\phi, m_{1}\right)=\left\{\begin{array}{lll}
\frac{f\left(\phi-m_{1}\right)-f(\phi)}{m_{1}} & \text { se } & m_{1}>0 \\
-f^{\prime}(\phi) & \text { se } & m_{1}=0
\end{array}\right.
$$

$J$ é contínua para $m_{1} \geq 0$ e para $0 \leq m_{1} \leq k_{1}$ temos

$$
u_{1}<u_{2}-k_{1}<u_{2}-m_{1}<u_{2} .
$$

Logo $J\left(u_{2}, m_{1}\right)>0, \forall m_{1} \in\left[0, k_{1}\right]$. Existe $\eta>0$ tal que $J\left(u_{2}, m_{1}\right)>2 \eta$ para $m_{1} \in\left[0, k_{1}\right]$.

Por continuidade, existe $\delta>0$ tal que $J\left(\phi, m_{1}\right)>\eta$ para $m_{1} \in\left[0, k_{1}\right]$ e $u_{2}-\delta<\phi<u_{2}$. Portanto, nesta faixa, temos

$$
f\left(\phi-q_{1}\right)-f(\phi) \geq \eta m_{1} .
$$

Pelos mesmos argumentos, podemos concluir que para $0 \leq \phi \leq \delta$ e $\phi>m_{1}$ também ocorre (1.86).

Portanto, como $\phi^{\prime}(\tau), \psi^{\prime}(\tau)>0, \quad \forall \tau \in \mathbb{R}$, e $n^{\prime}(t)>0$ existem constantes $\beta_{1}, \beta_{2}>0$ tais que

$$
-n^{\prime}(t) \psi^{\prime}(\tau)+m_{2}^{\prime}(t)+\varepsilon m_{1}(t)+\varepsilon \gamma m_{2}(t) \geq m_{2}^{\prime}(t)+\beta_{1} m_{1}(t)+\beta_{2} m_{2}(t) .
$$

Logo podemos concluir que

$$
\begin{aligned}
& N_{1}(\underline{y}, \underline{z}) \leq(\nu-\eta) m_{1}+m_{2} \\
& N_{2}(\underline{y}, \underline{z}) \geq \beta_{1} m_{1}+\left(\beta_{2}-\nu\right) m_{2} .
\end{aligned}
$$

Portanto, se

$$
\begin{aligned}
(\nu-\eta) k_{1}+k_{2} & \leq 0 \\
\beta_{1} k_{1}+\left(\beta_{2}-\nu\right) k_{2} & \geq 0
\end{aligned}
$$

então

$$
\nu \leq \frac{\eta k_{1}-k_{2}}{k_{1}}
$$

$\mathrm{e}$

$$
\nu \leq \frac{\beta k_{1}+\beta_{2} k_{2}}{k_{2}}
$$

Para $\eta>\frac{k_{2}}{k_{1}}$, seja

$$
\nu_{0}=\min \left\{\varepsilon \gamma, \frac{\eta k_{1}-k_{2}}{k_{1}}, \frac{\beta k_{1}+\beta_{2} k_{2}}{k_{2}}\right\} .
$$

Logo para todo $0 \leq \nu \leq \nu_{0}$,

$N_{1}(\underline{y}, \underline{z}) \leq 0$ e $N_{2}(\underline{y}, \underline{z}) \geq 0$ para todo $(x, t) \in \mathbb{R} \times \mathbb{R}^{+}$. 
A demonstração das desigualdades

$$
\begin{gathered}
y(x, t) \leq \phi\left(x-x_{2}\right)+k_{1} e^{-\nu t} \\
\psi\left(x-x_{2}\right)-k_{2} e^{-\nu t} \leq z(x, t) .
\end{gathered}
$$

é feita de forma análoga.

Estimativas de Schauder: Sejam $y$ e $z$ soluções de $(1.82)$ em $\mathbb{R} \times \mathbb{R}^{+}$, com $0 \leq y(x, t) \leq u_{2}$, $0 \leq z(x, t) \leq w_{2}$, e seja $F(x, t) \equiv f(y(x, t))-z(x, t)$. Para $x_{2}-x_{1}>2, t_{2}-t_{1}>1$ e $t_{1}>0$ definimos $Q \equiv\left[x_{1}, x_{2}\right] \times\left[t_{1}, t_{2}\right]$ e $Q^{\prime} \equiv\left[x_{1}+1, x_{2}-1\right] \times\left[t_{1}+1, t_{2}\right]$. Sejam

$$
\begin{gathered}
|y|_{0}^{Q} \equiv \sup _{Q}|y(x, t)|, \\
|y|_{1}^{Q} \equiv|y|_{0}^{Q}+|y|_{0}^{Q}, \\
|y|_{2}^{Q} \equiv|y|_{1}^{Q}+\left|y_{x x}\right|_{0}^{Q}+\left|y_{t}\right|_{0}^{Q} .
\end{gathered}
$$

Como $y$ é solução da equação $y_{t}-y_{x x}+c y_{x}-F(x, t)=0$ as estimativas de Schauder implicam que existe uma constante $l>0$ tal que

$$
\begin{gathered}
|y|_{1}^{Q} \leq l\left(|F|_{0}^{Q}+|y|_{0}^{Q}\right), \\
|y|_{2}^{Q^{\prime}} \leq l\left(|F|_{1}^{Q}+|y|_{0}^{Q}\right) \leq l\left(\left|F_{y}\right|_{0}^{Q}\left|y_{x}\right|_{0}^{Q}+\left|F_{z}\right|_{0}^{Q}\left|z_{x}\right|_{0}^{Q}+|y|_{0}^{Q}\right) .
\end{gathered}
$$

Para mais detalhes veja Fife e McLeod (1977) e Friedman (2013) . Como $F_{y}$ e $F_{z}$ são uniformemente limitadas em $\left[0, u_{2}\right] \times\left[0, w_{2}\right]$ obtemos limites em $\left|y_{t}\right|_{0}^{Q^{\prime}},\left|y_{x}\right|_{0}^{Q^{\prime}},\left|y_{x x}\right|_{0}^{Q^{\prime}}$ em termos de $|y|_{0}^{Q}$, $|F|_{0}^{Q},|z|_{0}^{Q},\left|z_{x}\right|_{0}^{Q}$.

Lema 1.4.2 Sob as hipóteses do Lema 1.4.1 existem constantes $C_{1}>0, k<0$ e $\sigma>0$, tais que $\frac{k}{2}+\sigma>0$ e satisfazem

$$
\left|u_{2}-y\right|,\left|w_{2}-z\right|,\left|y_{t}\right|,\left|y_{x}\right|,\left|y_{x x}\right|,\left|z_{x}\right|,\left|z_{t}\right|<C_{1}\left(e^{\left(-\frac{k}{2}-\sigma\right) x}+e^{-\nu t}\right), \quad \forall t \geq 0, x \geq 0
$$

$e$

$$
|y|,|z|,\left|y_{t}\right|,\left|y_{x}\right|,\left|y_{x x}\right|,\left|z_{x}\right|,\left|z_{t}\right|,<C_{1}\left(e^{\left(-\frac{k}{2}+\sigma\right) x}+e^{-\nu t}\right), \quad \forall t \geq 0, x \leq 0
$$

Demonstração. Primeiro vamos considerar o caso para $x \geq 0$.

Na Subseção 1.1.2 vimos que a matriz do sistema linear

$$
\frac{d}{d \xi}\left(\begin{array}{c}
\phi \\
\phi^{\prime} \\
\psi
\end{array}\right)=\left(\begin{array}{ccc}
0 & 1 & 0 \\
-f^{\prime}\left(u_{2}\right) & c & 1 \\
\frac{\varepsilon}{c} & 0 & -\frac{\varepsilon \gamma}{c}
\end{array}\right)\left(\begin{array}{l}
\phi \\
\phi^{\prime} \\
\psi
\end{array}\right)
$$

possui três raízes satisfazendo $r_{3}<r_{2}<0<r_{1}$.

Escrevendo o polinômio característico da matriz acima na forma fatorada

$$
p(r)=\left(r-r_{3}\right)\left(r^{2}+k r+l\right)
$$

segue que $r_{1,2}=\frac{-k \pm \sqrt{k^{2}-4 l}}{2}$. 
Seja $\sigma=\frac{\sqrt{k^{2}-4 l}}{2}$ então

$$
r_{1}=-\frac{k}{2}+\sigma \quad \text { e } \quad r_{2}=-\frac{k}{2}-\sigma .
$$

Usando a relação (1.4) e as equações de Girard, temos

$$
r_{1}+r_{2}+r_{3}=\left(c-\frac{\varepsilon \gamma}{c}\right) .
$$

Como $r_{1}+r_{2}=-k$ então $-k=c-\frac{\varepsilon \gamma}{c}-r_{3}$. Como $0>-\frac{\varepsilon \gamma}{c}>r_{3}$ e $c>0$ segue que $k<0$.

Como $(\phi, \psi) \rightarrow\left(u_{2}, w_{2}\right)$ exponencialmente quando $x \rightarrow \infty$, então existe um valor $\bar{K}_{1}$ tal que

$$
\max \left\{\left|u_{2}-\phi\right|,\left|w_{2}-\psi\right|\right\}<\bar{K}_{1} e^{r_{2} x}=\bar{K}_{1} e^{\left(-\frac{k}{2}-\sigma\right) x} \quad \text { para } \quad x \geq 0 .
$$

Logo, usando o Lema 1.4.1 obtemos

$$
\begin{aligned}
\left|u_{2}-y\right| & \leq\left|u_{2}-\phi\right|+|\phi-y| \leq \bar{K}_{1} e^{\left(-\frac{k}{2}-\sigma\right) x}+k_{1} e^{-\nu t} \\
& <K_{1}\left(e^{\left(-\frac{k}{2}-\sigma\right) x}+e^{-\nu t}\right)
\end{aligned}
$$

e também

$$
\left|w_{2}-z\right|<K_{1}\left(e^{\left(-\frac{k}{2}-\sigma\right) x}+e^{-\nu t}\right) .
$$

Sejam $x_{2}-x_{1}>2, t_{2}-t_{1}>1$ e $t_{1}>0$, definimos $Q \equiv\left[x_{1}, x_{2}\right] \times\left[t_{1}, t_{2}\right]$. As soluções $y$ e $z$ quando avaliadas no retângulo $Q$ ficam dentro do retângulo $R=\left[0, u_{2}\right] \times\left[0, w_{2}\right]$. Logo

$$
\begin{aligned}
|F|_{0}^{Q}=\sup _{Q}|f(y)-z| & =\sup _{Q}\left|f(y)-f\left(u_{2}\right)+f\left(u_{2}\right)+w_{2}-z-w_{2}\right| \\
& \leq \sup _{y^{*} \in\left[0, u_{2}\right]}\left|f^{\prime}\left(y^{*}\right)\right|\left|u_{2}-y\right|+\left|w_{2}-z\right| \\
& \leq K_{2}\left(e^{\left(-\frac{k}{2}-\sigma\right) z}+e^{-\mu t}\right) .
\end{aligned}
$$

Pelas estimativas de Schauder,

$$
\begin{aligned}
|y|_{0}^{Q}+\left|y_{x}\right|_{0}^{Q} & \leq l\left(|F|_{0}^{Q}+|y|_{0}^{Q}\right) \\
\left|y_{x}\right|_{0}^{Q} & \leq K_{3}\left(e^{\left(-\frac{k}{2}-\sigma\right) x}+e^{-\mu t}\right) .
\end{aligned}
$$

Estimativa para $z_{x}$ e $z_{t}$ : Como

$$
\begin{aligned}
\varepsilon(y-\gamma z) & =\varepsilon\left(y-u_{2}+u_{2}-\gamma\left(z-w_{2}+w_{2}\right)\right) \\
& =\varepsilon\left(y-u_{2}\right)+\varepsilon \gamma\left(w_{2}-z\right)+\varepsilon\left(u_{2}-\gamma w_{2}\right)
\end{aligned}
$$

$\log 0$

$$
\begin{aligned}
& \left|z_{t}\right| \leq \varepsilon\left|y-u_{2}\right|+\varepsilon \gamma\left|w_{2}-z\right| \\
& \left|z_{t}\right| \leq K\left(e^{\left(-\frac{k}{2}-\sigma\right) x}+e^{-\mu t}\right) .
\end{aligned}
$$

e também

$$
\left|z_{x}\right| \leq K\left(e^{\left(-\frac{k}{2}-\sigma\right) x}+e^{-\mu t}\right)
$$


E finalmente as estimativas para as derivadas de $u$

$$
\begin{aligned}
|y|_{2}^{Q^{\prime}} & \leq l\left(\left|F_{y}\right|_{0}^{Q}\left|y_{x}\right|_{0}^{Q}+\left|F_{z}\right|_{0}^{Q}\left|z_{x}\right|_{0}^{Q}+|y|_{0}^{Q}\right) \\
|y|_{0}^{Q}+\left|y_{x}\right|_{0}^{Q}+\left|y_{x x}\right|_{0}^{Q}+\left|y_{t}\right|_{0}^{Q} & \leq K_{4}\left(e^{\left(-\frac{k}{2}-\sigma\right) x}+e^{-\nu t}\right) .
\end{aligned}
$$

Para $x \leq 0$, sabemos que $(\phi, \psi)$ converge exponencialmente para $(0,0)$ quando $x \rightarrow-\infty$ e portanto existe $\bar{K}_{1}>0$ tal que

$$
\max \{|\phi|,|\psi|\}<\bar{K}_{1} e^{r_{1} z}=\bar{K}_{1} e^{\left(-\frac{k}{2}+\sigma\right) x} \quad \text { para } x \leq 0 .
$$

e as demais estimativas seguem de forma análoga pelas estimativas de Schauder. 


\section{Capítulo 2}

\section{Aproximando ondas viajantes}

\subsection{Definição do problema não local}

Dado que existe $c^{*}>0$ e uma única solução onda viajante $(\phi(\xi), \psi(\xi))$, a menos de translação, do problema (1) que satisfaz (6) e que esta solução é assintoticamente estável, nosso interesse agora é obter uma caracterização do perfil $(\phi, \psi)$ e da velocidade de propagação $c^{*}$. Na literatura é muito comum usar métodos numéricos para aproximar ondas viajantes, mas para isso é necessário fixar um domínio computacional finito. Estudos anteriores ${ }^{1}$ mostraram que, o comportamento do problema em um intervalo finito pode ter um comportamento completamente diferente do sistema original quando truncado neste mesmo intervalo, embora sejam equivalentes em $\mathbb{R}$.

Uma saída para solucionar este problema consiste em aproximar o perfil $(\phi, \psi)$ por equilíbrios da equação local

$$
\left\{\begin{array}{l}
u_{t}=u_{x x}-c^{*} u_{x}+f(u)-w \\
w_{t}=-c^{*} w_{x}+\varepsilon(u-\gamma w)
\end{array}\right.
$$

já que soluções ondas viajantes $(\phi, \psi)$ podem ser vistas como equilíbrios de tais equações. Porém, tanto $(\phi, \psi)$ quanto $c^{*}$ são desconhecidas, o que dificulta a utilização dessa abordagem.

Queremos calcular tanto perfil $(\phi, \psi)$ quanto a velocidade $c^{*}$ ao mesmo tempo. Vamos adotar a proposta utilizada em Arrieta et al. (2011) para aproximar ondas viajantes no caso da equação de reação-difusão unidimensional.

A título de motivação das equações que serão consideradas a seguir, vamos determinar expressões para $c^{*}$. Multiplicando por $\phi^{\prime}$ na primeira equação do sistema (4) e depois integrando ao longo de $\mathbb{R}$, usando o fato que $\phi^{\prime}( \pm \infty)=0$, obtemos

$$
c^{*}=\frac{\left\langle f(\phi)-\psi, \phi^{\prime}\right\rangle}{\left\langle\phi^{\prime}, \phi^{\prime}\right\rangle}
$$

onde $\langle\cdot, \cdot\rangle$ é o produto interno em $L^{2}(\mathbb{R})$.

Por outro lado, se multiplicarmos $\psi^{\prime}$ na segunda equação do sistema (4) e depois integrar em $\mathbb{R}$ concluímos que

$$
c^{*}=\frac{\left\langle\varepsilon(\phi-\gamma \psi), \psi^{\prime}\right\rangle}{\left\langle\psi^{\prime}, \psi^{\prime}\right\rangle} .
$$

Observe que essas duas definições são equivalentes pois

$$
\frac{\left\langle f(\phi)-\psi, \phi^{\prime}\right\rangle}{\left\langle\phi^{\prime}, \phi^{\prime}\right\rangle}-\frac{\left\langle\varepsilon(\phi-\gamma \psi), \psi^{\prime}\right\rangle}{\left\langle\psi^{\prime}, \psi^{\prime}\right\rangle}=-\frac{\left\langle\phi^{\prime \prime}, \phi^{\prime}\right\rangle}{\left\langle\phi^{\prime}, \phi^{\prime}\right\rangle}+c^{*} \frac{\left\langle\phi^{\prime}, \phi^{\prime}\right\rangle}{\left\langle\phi^{\prime}, \phi^{\prime}\right\rangle}-c^{*} \frac{\left\langle\psi^{\prime}, \psi^{\prime}\right\rangle}{\left\langle\psi^{\prime}, \psi^{\prime}\right\rangle}=0 .
$$

\footnotetext{
${ }^{1}$ Veja Beyn et al. (2014)
} 
Fazendo uma mudança de variáveis (2.1) pode ser escrita na forma

$$
c^{*}=\frac{F\left(u_{2}\right)-\int_{-\infty}^{\infty} \phi^{\prime}(x) \psi(x) d x}{\int_{-\infty}^{\infty} \phi^{\prime}(x)^{2} d x}=\frac{F\left(u_{2}\right)-\left\langle\phi^{\prime}, \psi\right\rangle}{\left\langle\phi^{\prime}, \phi^{\prime}\right\rangle},
$$

onde $F\left(u_{2}\right)=\int_{0}^{u_{2}} f(s) d s$.

Substituindo a expressão de $c^{*}$ encontrados acima em (4), concluímos que $(\phi, \psi)$ é solução do sistema não local

$$
\left\{\begin{array}{r}
\phi^{\prime \prime}(x)-\frac{F\left(u_{2}\right)-\left\langle\phi^{\prime}, \psi\right\rangle}{\left\langle\phi^{\prime}, \phi^{\prime}\right\rangle} \phi^{\prime}(x)+f(\phi(x))-\psi(x)=0 \\
-\frac{F\left(u_{2}\right)-\left\langle\phi^{\prime}, \psi\right\rangle}{\left\langle\phi^{\prime}, \phi^{\prime}\right\rangle} \psi^{\prime}(x)+\varepsilon(\phi(x)-\gamma \psi(x))=0 .
\end{array}\right.
$$

Por outro lado, a mudança de variáveis

$$
\left\{\begin{array}{l}
u(x, t)=p(x+\eta(t), t) \\
w(x, t)=q(x+\eta(t), t)
\end{array}\right.
$$

transforma (1) no sistema

$$
\begin{cases}p_{t}=p_{x x} & -\eta^{\prime}(t) p_{x}+f(p)-q \\ q_{t}= & -\eta^{\prime}(t) q_{x}+\varepsilon(p-\gamma q) .\end{cases}
$$

A simples comparação de (2.4) com (2.6) sugere que, para que (2.6) seja uma aproximação razoável de (1), devemos escolher as função $\eta(t)$ de tal forma que $\eta^{\prime}(t) \rightarrow c^{*}$ quando $t \rightarrow+\infty$, $i=1,2$.

Sendo assim, podemos definir

$$
\eta^{\prime}(t)=\frac{A-\left\langle q(\cdot, t), p_{x}(\cdot, t)\right\rangle}{\left\langle p_{x}(\cdot, t), p_{x}(\cdot, t)\right\rangle}
$$

ou, equivalentemente,

$$
\eta(t)=\int_{0}^{t} \frac{A-\left\langle q(\cdot, s), p_{x}(\cdot, s)\right\rangle}{\left\langle p_{x}(\cdot, s), p_{x}(\cdot, s)\right\rangle} d s
$$

onde $A=\int_{0}^{u_{2}} f(s) d s$.

Consideremos o problema de Cauchy para o sistema não local

$$
\begin{cases}p_{t}=p_{x x}-\frac{A-\left\langle q, p_{x}\right\rangle}{\left\langle p_{x}, p_{x}\right\rangle} p_{x}+f(p)-q, & \\ q_{t}=-\frac{A-\left\langle q, p_{x}\right\rangle}{\left\langle p_{x}, p_{x}\right\rangle} q_{x}+\varepsilon(p-\gamma q), & -\infty<x<\infty, \quad t>0 .\end{cases}
$$

Como é de se esperar, e provaremos nos próximos resultados, as soluções de (2.9) estão intimamente relacionadas com as soluções de (1). 


\subsection{Propriedades das soluções do problema local}

No que segue, vamos admitir que as condições iniciais $u_{0}, w_{0}$ de (1) satisf azem $0 \leq u_{0}(x) \leq u_{2}$, $0 \leq w_{0}(x) \leq w_{2}$ e mais as hipóteses do Lema 1.4.1:

$$
\begin{array}{ll}
\lim _{x \rightarrow-\infty} \sup u_{0}(x)<u_{1} & \lim _{x \rightarrow \infty} \inf u_{0}(x)>u_{1} . \\
\lim _{x \rightarrow-\infty} \sup w_{0}(x)<w_{1} & \lim _{x \rightarrow \infty} \inf w_{0}(x)>w_{1} .
\end{array}
$$

Nessas condições, pelos Teorema 1.3.3 e Lema 1.4.1, a solução de (1) com condições iniciais

$$
u(x, 0)=u_{0}(x) \quad \text { e } \quad w(x, 0)=w_{0}(x)
$$

converge para uma translação de $(\phi, \psi)$.

Seja $u_{0} \in L^{p}(\mathbb{R}) \cap C^{2+\alpha}(\mathbb{R})$ e $w_{0} \in L^{p}(\mathbb{R}) \cap C^{\alpha}(\mathbb{R})$ com $p \in[1, \infty]$ e $\alpha \in(0,1)$. A existência e unicidade de uma solução clássica global do problema (1)-(2.10) em espaços $L^{p}(\mathbb{R})$ podem ser encontrados em Rothe (1984) e Rauch (1976).

Pela limitação das condições iniciais feita acima concluímos que existe uma única solução limitada tal que $(u, w) \in\left[0, u_{2}\right] \times\left[0, w_{2}\right]$, para todo $x \in \mathbb{R}$ e $t \geq 0$. Veja, por exemplo, Pao (2012) ou Rothe (1984) para tal conclusão.

No que segue, precisaremos de algumas estimativas sobre $u_{x}, u_{x x}$ e $w_{x}$ em alguns espaços $L^{p}$. Considere

$$
\mathbb{X}^{p}=\left\{u \in L_{\mathrm{loc}}^{p}(\mathbb{R}): \partial_{x} u \in L^{p}(\mathbb{R})\right\}, \quad p \geq 1
$$

Se $\left(u_{0}, w_{0}\right) \in \mathbb{X}^{p} \times \mathbb{X}^{p}$, pelo Teorema 2.2 de Rauch e Smoller (1978) o problema

$$
\begin{cases}h_{t}=h_{x x}+f^{\prime}(u(x, t)) h-g, & -\infty<x<\infty \\ g_{t}=\varepsilon(h-\gamma g), & \end{cases}
$$

com condições iniciais

$$
h(x, 0)=\partial_{x} u_{0} \in \mathbb{X}^{p} \text { e } g(x, 0)=\partial_{x} w_{0} \in \mathbb{X}^{p}
$$

tem única solução clássica em $L^{p}(\mathbb{R}) \times L^{p}(\mathbb{R})$, ou seja, $u_{x}, w_{x} \in L^{p}(\mathbb{R})$ para $p \geq 1$. Logo o problema (1)-(2.10) tem uma única solução clássica e limitada em $\mathbb{X}^{p} \times \mathbb{X}^{p}$ para $p \geq 1$.

Proposição 2.2.1 Sob as condições acima, são verdadeiras as seguintes afirmaçôes:

(i) existe $\beta>0$ tal que $\left\|u_{x}(\cdot, t)\right\|_{L^{2}(\mathbb{R})} \geq \beta$ e $\left\|w_{x}(\cdot, t)\right\|_{L^{2}(\mathbb{R})} \geq \beta$ para todo $t>0$;

(ii) Seja $\gamma>\frac{1}{\max \left\{f^{\prime}(0), f^{\prime}\left(u_{2}\right)\right\}}$ existe $C>0$ tal que $\left\|u_{x}(\cdot, t)\right\|_{L^{1}(\mathbb{R})} \leq C$ e $\left\|w_{x}(\cdot, t)\right\|_{L^{1}(\mathbb{R})} \leq C$ para todo $t>0$;

(iii) $u_{x x} \in L^{1}(\mathbb{R})$ e existe $C>0$ tal que $\left\|u_{x x}(\cdot, t)\right\|_{L^{1}(\mathbb{R})} \leq C$, para todo $t>1$.

Demonstração. $(i)$ Pelo Teorema 1.3.3, as soluções $(u, w)$ se aproximam exponencialmente da solução onda viajante e portanto $\liminf _{t \rightarrow \infty}\left\|u_{x}(\cdot, t)\right\|_{L^{2}(\mathbb{R})}>0$, ou seja, existem $T_{1}$ e $\beta_{1} \operatorname{com}\left\|u_{x}(\cdot, t)\right\|_{L^{2}(\mathbb{R})} \geq$ $\beta_{1}$ para todo $t \geq T_{1}$.

Suponha que para todo $t \in\left(0, T_{1}\right),\left\|u_{x}(\cdot, t)\right\|_{L^{2}(\mathbb{R})}=0$. Logo $u_{x}(\cdot, t)=0$ e portanto $u(\cdot, t)=C(t)$ para todo $t \in\left(0, T_{1}\right]$. Como $u(x, 0)=u_{0}(x)$, pela unicidade de solução $u(\cdot, t)=u_{0}(x)=C(t)=K$ para todo $x \in \mathbb{R}$ e $t \geq 0$ o que é um absurdo, logo $\left\|u_{x}(\cdot, t)\right\|_{L^{2}(\mathbb{R})}>0$ para todo $t \in\left[0, T_{1}\right]$.

Considerando a função contínua $t \in\left[0, T_{1}\right] \mapsto\left\|u_{x}(\cdot, t)\right\|_{L^{2}(\mathbb{R})}$, existe um $\beta>0$ tal que

$$
\left\|u_{x}(\cdot, t)\right\|_{L^{2}(\mathbb{R})} \geq \beta
$$

para todo $t \in\left[0, T_{1}\right]$. 
O mesmo raciocínio usamos para provar que $\left\|w_{x}(\cdot, t)\right\|_{L^{2}(\mathbb{R})} \geq \beta>0$.

(ii) O resultado que queremos provar é equivalente a mostrar a limitação uniforme de $\left\|y_{x}(\cdot, t)\right\|_{L^{1}(\mathbb{R})}$ e $\left\|z_{x}(\cdot, t)\right\|_{L^{1}(\mathbb{R})}$, onde

$$
y(x+c t, t)=u(x, t) \text { e } z(x+c t, t)=w(x, t) .
$$

Se $h=u_{x}$ e $g=w_{x}$ então $h$ e $g$ satisfazem o sistema

$$
\begin{cases}h_{t}=h_{x x}+f^{\prime}(y(x, t)) h-g & -\infty<x<\infty \\ g_{t}=\varepsilon(h-\gamma g) & \end{cases}
$$

Multiplicando a primeira equação por $\operatorname{sign}(h)$ e a segunda por $\operatorname{sign}(g)$, temos

$$
\begin{aligned}
& |h|_{t} \leq|h|_{x x}+c|h|_{x}+f^{\prime}(y)|h|+|g|, \\
& |g|_{t} \leq c|g|_{x}+\varepsilon|h|-\varepsilon \gamma|g| .
\end{aligned}
$$

Pelo Teorema 1.3.3 e por $f^{\prime}$ ser contínua podemos estimar

$$
\left|f^{\prime}(u(x, t))-f^{\prime}\left(\phi\left(x-x_{0}\right)\right)\right| \leq C\left|u(x, t)-\phi\left(x-x_{0}\right)\right| \leq K e^{-\omega t} .
$$

Como $f^{\prime}(0), f^{\prime}\left(u_{2}\right)<0$ existem $L>0$ e $t_{0}>0$, suficientemente grande, tal que

$$
f^{\prime}(u(x, t)) \leq f^{\prime}\left(\phi\left(x-x_{0}\right)\right)+k_{1} e^{-\nu t} \leq-\beta<0 \quad \text { para todo }|x| \geq L \text { e } t \geq t_{0},
$$

para algum $\beta>0$.

Integrando no conjunto $\{x \in \mathbb{R} ;|x| \geq L\}$, obtemos

$$
\begin{aligned}
& \frac{d}{d t} \int_{|x| \geq L}|h(x, t)| d x \leq \int_{|x| \geq L}\left(|h|_{x x}+c|h|_{x}\right) d x-\beta \int_{|x| \geq L}|h(x, t)| d x+\int_{|x| \geq L}|g(x, t)| d x, \\
& \frac{d}{d t} \int_{|x| \geq L}|g(x, t)| d x \leq c \int_{|x| \geq L}|g|_{x} d x+\varepsilon \int_{|x| \geq L}|h(x, t)| d x-\varepsilon \gamma \int_{|x| \geq L}|g(x, t)| d x,
\end{aligned}
$$

Usando as estimativas do Lema 1.4.2, concluímos que existem $C>0$ e $k>0$ tais que

$$
\begin{aligned}
\frac{d}{d t} \int_{|x| \geq L}|h(x, t)| d x & \leq \int_{|x| \geq L}|h|_{x x} d x+c\left(\limsup _{M \rightarrow \pm \infty}|h(M, t)|+|h( \pm L, t)|\right)-\beta \int_{|x| \geq L}|h(x, t)| d x \\
+\int_{|x| \geq L}|g(x, t)| d x & \\
& \left.\leq C_{1} e^{-k_{1} t}+\limsup _{M \rightarrow \pm \infty}\left|h_{x}(M, t)\right|+\left|h_{x}( \pm L, t)\right|\right)-\beta \int_{|x| \geq L}|h(x, t)| d x \\
& +\int_{|x| \geq L}|g(x, t)| d x \\
& \leq C e^{-k t}-\beta \int_{|x| \geq L}|h(x, t)| d x+\int_{|x| \geq L}|g(x, t)| d x
\end{aligned}
$$

e também

$$
\frac{d}{d t} \int_{|x| \geq L}|g(x, t)| d x \leq C e^{-k t}+\varepsilon \int_{|x| \geq L}|h(x, t)| d x-\varepsilon \gamma \int_{|x| \geq L}|g(x, t)| d x .
$$


Sejam $m(t)=\int_{|x| \geq L}|h(x, t)| d x$ e $n(t)=\int_{|x| \geq L}|g(x, t)| d x$. Note que

$$
\frac{d}{d t}\left(\begin{array}{c}
m(t) \\
n(t)
\end{array}\right) \leq\left(\begin{array}{cc}
-\beta & 1 \\
\varepsilon & -\varepsilon \gamma
\end{array}\right)\left(\begin{array}{c}
m(t) \\
n(t)
\end{array}\right)+C\left(\begin{array}{c}
e^{-k t} \\
e^{-k t}
\end{array}\right)
$$

Considere o sistema $x^{\prime}=M x+C e^{-k t}$, onde

$$
M=\left(\begin{array}{cc}
-\beta & 1 \\
\varepsilon & -\varepsilon \gamma
\end{array}\right) .
$$

A matriz $\mathrm{M}$ tem dois autovalores reais tais que $r_{1}<r_{2}<0$. Como $m(t)>0$ e $n(t)>0$, para todo $t \geq t_{0}$, existem constantes $D>0$ e $d>0$ tais que

$$
m(t)<D e^{-d t} \leq K \quad \text { e } \quad n(t)<D e^{-d t} \leq K \quad \text { para } t \geq t_{0} .
$$

Logo, para todo $t \geq t_{0}$, usando as estimativas do Lema 1.4.2, temos

$$
\begin{aligned}
\int_{\mathbb{R}}|h(x, t)| d x & =\int_{|x| \geq L}|h(x, t)| d x+\int_{|x| \leq L}|h(x, t)| d x \\
& \leq K+2 L \sup _{x \in[-L, L]}|h(x, t)| \leq C .
\end{aligned}
$$

De forma análoga obtemos $\int_{\mathbb{R}}|g(x, t)| d x \leq C$.

Para obtermos a limitação em $\left(0, t_{0}\right]$ recordemos que $U=\left(u_{x}, w_{x}\right)$ é solução de (2.13)-(2.12) e pelo Teorema 2.2 de Rauch e Smoller (1978) tem-se a estimativa: $\|U(t)\|_{L^{p}(\mathbb{R})} \leq k e^{c t}\left\|U_{0}\right\|_{L^{p}(\mathbb{R})}$.

(iii) Considerando $h=u_{x}$ como solução de (2.11)-(2.12) por propriedades de regularização é possível $\operatorname{mostrar}^{2}$ que $\left\|h_{x}(\cdot, t+1)\right\|_{L^{1}(\mathbb{R})} \leq C\|h(\cdot, t)\|_{L^{1}(\mathbb{R})}$ e pelo item (ii) desta proposição o resultado é imediato.

\subsection{Propriedades das soluções do problema não local}

Nesta seção vamos usar as propriedades das soluções do problema local para verificar a boa colocação do problema não local e mostrar que o problema (2.9) tem uma família de ondas viajantes com velocidade $c=0$ cujo perfil converge exponencialmente para o perfil da onda viajante do problema original encontrada no Capítulo 1, assim como o termo $n^{\prime}(t) \rightarrow c^{*}$ quando $t \rightarrow \infty$.

Teorema 2.3.1 Seja $(u, w)$ a única solução clássica de (1) com condição inicial $\left(u_{0}, w_{0}\right)$ satisfazendo as condiçôes enunciadas acima. Definindo $(p, q)$ por

$$
\begin{aligned}
& p(x, t):=u(x-\eta(t), t), \\
& q(x, t):=w(x-\eta(t), t),
\end{aligned}
$$

com

$$
\eta(t)=\int_{0}^{t} \frac{A-\left\langle w(\cdot, s), u_{x}(\cdot, s)\right\rangle}{\left\langle u_{x}(\cdot, s), u_{x}(\cdot, s)\right\rangle} d s, \quad t>0
$$

então, $(p, q)$ está bem definida e é uma solução clássica de (2.9).

\footnotetext{
${ }^{2}$ Veja pag 13 de Arrieta et al. (2011)
} 
Demonstração. Como $(u, w)$ é solução clássica de (1), então satisfazem

(i) $u_{x}(\cdot, t), w_{x}(\cdot, t) \in L^{1}(\mathbb{R}) \cap L^{2}(\mathbb{R})$, para todo $t \geq 0$;

(ii) existe $\beta>0$ tal que $\left\|u_{x}(\cdot, t)\right\|_{L^{2}(\mathbb{R})} \geq \beta$, para todo $t \geq 0$, e, além disso, $0 \leq u(x, t) \leq u_{2}$ e $0 \leq w(x, t) \leq w_{2}$.

Por $(i)$

$$
\begin{aligned}
A-\left\langle w(\cdot, t), u_{x}(\cdot, t)\right\rangle & \left.\leq A+\int_{-\infty}^{\infty} \mid w(x, t)\right) \| u_{x}(x, t) \mid d x \\
& \leq C\left\|u_{x}(\cdot, t)\right\|_{L^{1}(\mathbb{R})}<\infty
\end{aligned}
$$

Portanto, o produto interno acima é convergente. Sejam

$$
\lambda(t):=\frac{A-\left\langle w(\cdot, t), u_{x}(\cdot, t)\right\rangle}{\left\langle u_{x}(\cdot, t), u_{x}(\cdot, t)\right\rangle} .
$$

Por $(i)$ e (ii) o denominador de (2.16) é finito e estritamente positivo, logo $t \longmapsto \lambda(t)$ define uma função contínua e limitada, para todo $t>0$. Portanto $\eta(t)$ e, consequentemente $p$ e $q$, estão bem definidas.

Pela invariância com respeito a translações do produto interno em $L^{2}(\mathbb{R})$, para cada $t \geq 0$ fixo,

$$
A-\left\langle q(\cdot, t), p_{x}(\cdot, t)\right\rangle=A-\left\langle w(\cdot, t), u_{x}(\cdot, t)\right\rangle .
$$

E também

$$
\left\|p_{x}(\cdot, t)\right\|_{L^{2}(\mathbb{R})}^{2}=\left\|u_{x}(\cdot, t)\right\|_{L^{2}(\mathbb{R})}^{2} .
$$

Então,

$$
\lambda(t)=\frac{A-\left\langle q(\cdot, t), p_{x}(\cdot, t)\right\rangle}{\left\langle p_{x}(\cdot, t), p_{x}(\cdot, t)\right\rangle}
$$

e $(p, q)$ satisfaz (2.9).

Teorema 2.3.2 Sob as condições do Teorema 1.3 .3 e, além disso, assuma que $u_{0}, w_{0} \in \mathbb{X}^{1} \cap \mathbb{X}^{2}$ o problema (2.9) é bem posto e sua solução $(p, q)$ é dada por (2.14)-(2.16). Seja $\omega$ do Teorema 1.3.3 e seja $\bar{\omega}<\omega$. Então, existem $x^{*} \in \mathbb{R}$ e constantes positivas $C_{1}$ e $C_{2}$ tais que

(i) Para $c^{*}$ velocidade de propagação do Teorema 1.1.2 e $\lambda_{1}$ em (2.17), temos

$$
\left|\lambda(t)-c^{*}\right| \leq C_{1} e^{-\bar{\omega} t}
$$

(ii) Para $(\phi, \psi)$ a única solução, solução onda viajante do problema (1), a menos de translação, temos

$$
\begin{aligned}
& \left|p(x, t)-\phi\left(x-x^{*}\right)\right| \leq C_{2} e^{-\bar{\omega} t}, \quad x \in \mathbb{R}, \quad t>0 \\
& \left|q(x, t)-\psi\left(x-x^{*}\right)\right| \leq C_{2} e^{-\bar{\omega} t},
\end{aligned}
$$

para alguma constante $C_{2}>0$ e $x^{*} \in \mathbb{R}$.

Demonstração. (i) Inicialmente, temos

$$
\left|\lambda(t)-c^{*}\right|=\left|\frac{A-\left\langle q, p_{x}\right\rangle}{\left\langle p_{x}, p_{x}\right\rangle}-c^{*}\right|=\frac{1}{\left\|p_{x}\right\|_{2}^{2}}\left|A-\left\langle q, p_{x}\right\rangle-c^{*}\left\|p_{x}\right\|_{2}^{2}\right| .
$$

Logo, pelo item (i) da Proposição 2.2.1, para algum $C>0$ temos

$$
\left|\lambda(t)-c^{*}\right| \leq C\left|A-\left\langle q, p_{x}\right\rangle-c^{*}\left\|p_{x}\right\|_{2}^{2}\right| .
$$


Seja $(y, z)$ como em $(1.81)$

$$
A-\left\langle q, p_{x}\right\rangle-c^{*}\left\|p_{x}\right\|_{2}^{2}=A-\left\langle z, y_{x}\right\rangle-c^{*}\left\|y_{x}\right\|_{2}^{2}
$$

e para $(\phi, \psi)=\left(\phi\left(x-x_{0}\right), \psi\left(x-x_{0}\right)\right)$, com $x_{0}$ do Teorema 1.3.3 usando a fórmula (2.3) obtemos

$$
\begin{aligned}
A-\left\langle q, p_{x}\right\rangle-c^{*}\left\|p_{x}\right\|_{2}^{2} & =A-\left\langle z, y_{x}\right\rangle-c^{*}\left\|y_{x}\right\|_{2}^{2} \\
& =A-\left\langle z, y_{x}\right\rangle-c^{*}\left\|y_{x}\right\|_{2}^{2}-A+\left\langle\psi, \phi^{\prime}\right\rangle+c^{*}\left\|\phi^{\prime}\right\|_{2}^{2} .
\end{aligned}
$$

Portanto,

$$
A-\left\langle q, p_{x}\right\rangle-c^{*}\left\|p_{x}\right\|_{2}^{2}=\left\langle\psi-z, y_{x}\right\rangle+\left\langle\psi, \phi^{\prime}-y_{x}\right\rangle-c^{*}\left\langle y_{x}-\phi^{\prime}, y_{x}+\phi^{\prime}\right\rangle .
$$

Vamos analisar cada elemento da soma acima separadamente.

Na primeira parcela de (2.20), concluímos pelo Teorema 1.3.3 e pelo item (ii) da Proposição 2.2.1 que existe constante $\tilde{C}>0$ tal que

$$
\left|\left\langle\psi-z, y_{x}\right\rangle\right| \leq \tilde{C} e^{-\omega t}
$$

Na segunda parcela de (2.21), usando integração por partes,

$$
\begin{aligned}
\left|\left\langle\psi, \phi^{\prime}-y_{x}\right\rangle\right| & =\lim _{L \rightarrow \infty}\left|\int_{-L}^{L} \psi\left(\phi^{\prime}-y_{x}\right) d x\right| \\
& \leq \limsup _{L \rightarrow \infty}|\psi(\phi-y)|_{x=-L}^{x=L}-\int_{-L}^{L}(\phi-y) \psi^{\prime} d x \mid \\
& \leq \limsup _{L \rightarrow \infty}|\psi(\phi-y)|_{x=-L}^{x=L}+\left|\left\langle\psi^{\prime}, \phi-y\right\rangle\right| .
\end{aligned}
$$

A análise da segunda parcela é então dividida em duas partes:

$1^{\mathrm{a}}$ parte: Como $\psi$ é limitada

$$
\limsup _{L \rightarrow \infty}|\psi(\phi-y)|_{x=-L}^{x=L} \mid \leq \limsup _{L \rightarrow \infty}\{C|\phi(L)-y(L, t)|+C|\phi(-L)-y(-L, t)|\} .
$$

Pelo Lema 1.4.2,

$$
\begin{aligned}
|\phi(L)-y(L, t)| & \leq\left|\phi(L)-u_{2}\right|+\left|u_{2}-y(L, t)\right| \\
& \leq K_{1} e^{-\nu t}+C_{1}\left(e^{\left(-\frac{k}{2}-\sigma\right) L}+e^{-\nu t}\right), \quad \forall t \geq 0
\end{aligned}
$$

e também

$$
|\phi(-L)-y(-L, t)| \leq C_{2}\left(e^{-\left(-\frac{k}{2}+\sigma\right) L}+e^{-\nu t}\right)+K_{2} e^{-\nu t}, \quad \forall t \geq 0 .
$$

Portanto, existem $C>0$ e $\sigma *>0$ tais que

$$
\max \{|\phi(L)-y(L, t)|,|\phi(-L)-y(-L, t)|\} \leq C\left(e^{-\sigma^{*} L}+e^{-\nu t}\right) .
$$

E assim,

$$
\limsup _{L \rightarrow \infty}|\psi(\phi-y)|_{x=-L}^{x=L} \mid \leq \lim _{L \rightarrow \infty} C\left(e^{-\sigma^{*} L}+e^{-\nu t}\right) \leq \bar{C} e^{-\nu t}
$$

$2^{\mathrm{a}}$ parte: Como $\psi^{\prime}( \pm \infty)=0$ e pelo Teorema 1.3.3 temos

$$
\left|\left\langle\psi^{\prime}, \phi-y\right\rangle\right| \leq C \sup _{x \in \mathbb{R}}|\phi-y| \leq \tilde{C} e^{-\omega t}
$$


Logo, existe $\tilde{\tilde{C}}>0$ tal que

$$
\left|\left\langle\psi, \phi^{\prime}-y_{x}\right\rangle\right| \leq \tilde{\tilde{C}}\left(e^{-\nu t}+e^{-\omega t}\right) .
$$

Agora vamos analisar a terceira e última parcela de (2.20).

Fazendo integração por partes

$$
\begin{aligned}
\left|\left\langle y_{x}-\phi^{\prime}, y_{x}+\phi^{\prime}\right\rangle\right| & \leq \lim _{L \rightarrow \infty}\left|\int_{-L}^{L}\left(y_{x}-\phi^{\prime}\right)\left(y_{x}+\phi^{\prime}\right) d x\right| \\
& \leq \limsup _{L \rightarrow \infty}\left|\left(y_{x}+\phi^{\prime}\right)(y-\phi)\right|_{x=-L}^{x=L}-\int_{-L}^{L}(y-\phi)\left(y_{x x}+\phi^{\prime \prime}\right) d x \mid \\
& \leq \limsup _{L \rightarrow \infty}\left|\left(y_{x}+\phi^{\prime}\right)(y-\phi)\right|_{x=-L}^{x=L}+\left|\left\langle y-\phi, y_{x x}+\phi^{\prime \prime}\right\rangle\right|
\end{aligned}
$$

Pelo Lema 1.4.2, existem constantes $C^{\prime \prime \prime}>0$ e $\sigma^{* *}>0$ tais que

$$
\limsup _{L \rightarrow \infty}\left|\left(y_{x}+\phi^{\prime}\right)(y-\phi)\right|_{x=-L}^{x=L} \leq \lim _{L \rightarrow \infty} C^{\prime \prime \prime}\left(e^{-\nu t}+e^{-\sigma^{* *}}\right) \leq C e^{-\nu t} .
$$

Pelo Teorema 1.3.3 e pelo item (iii) da Proposição 2.2.1,

$$
\begin{aligned}
\left|\left\langle y-\phi, y_{x x}+\phi^{\prime \prime}\right\rangle\right| & \leq \int_{-\infty}^{\infty}|y-\phi|\left|y_{x x}+\phi^{\prime \prime}\right| d x \\
& \leq \sup _{x \in \mathbb{R}}\left|y(x, t)-\phi\left(x-x_{0}\right)\right|\left\|y_{x x}+\phi^{\prime \prime}\right\|_{L^{1}(\mathbb{R})} \\
& \leq \tilde{C} e^{-\omega t} .
\end{aligned}
$$

Portanto, existe constante $\tilde{\tilde{C}}>0$ tal que

$$
\left|\left\langle y_{x}-\phi^{\prime}, y_{x}+\phi^{\prime}\right\rangle\right| \leq \tilde{\tilde{\tilde{C}}}\left(e^{-\nu t}+e^{-\omega t}\right) .
$$

Concluímos de (2.21), (2.22) e (2.23) que $\left|\lambda(t)-c^{*}\right| \leq \dot{C} e^{-\nu t}+\ddot{C} e^{-\omega t}$. Sejam $\bar{\omega}<\min \{\nu, \omega\}$ e $C_{1}>\max \{2 \dot{C}, 2 \ddot{C}\}$ então

$$
\left|\lambda(t)-c^{*}\right| \leq C_{1} e^{-\bar{\omega} t}
$$

(ii) Devido a (2.18) sabemos que

$$
\begin{aligned}
\int_{0}^{\infty}\left(\lambda(s)-c^{*}\right) d s & \leq \lim _{t \rightarrow \infty} \int_{0}^{t}\left|\lambda(s)-c^{*}\right| d s \\
& \leq \lim _{t \rightarrow \infty} \int_{0}^{t} C_{1} e^{-\bar{\omega} s} d s \\
& \leq \lim _{t \rightarrow \infty}\left(-\frac{C_{1}}{\bar{\omega}} e^{-\bar{\omega} t}+\frac{C_{1}}{\bar{\omega}}\right)<\infty
\end{aligned}
$$


Seja $I=\int_{0}^{\infty}\left(\lambda(s)-c^{*}\right) d s, I \in \mathbb{R}$, e lembrando que $\eta^{\prime}=\lambda$, obtemos

$$
\begin{aligned}
\left|-\eta(t)+c^{*} t+I\right| & =\left|-\left(\int_{0}^{t} \lambda(s) d s-c^{*} t\right)+\int_{0}^{\infty} \lambda(s)-c^{*} d s\right| \\
& =\left|-\int_{0}^{t}\left(\lambda(s)-c^{*}\right) d s+\int_{0}^{\infty}\left(\lambda(s)-c^{*}\right) d s\right| \\
& =\left|\int_{t}^{\infty}\left(\lambda(s)-c^{*}\right) d s\right| \\
& \leq \int_{t}^{\infty} C_{1} e^{-\bar{\omega} s} d s \\
& \leq \tilde{C} e^{-\bar{\omega} t}
\end{aligned}
$$

para alguma constante $\tilde{C}>0$.

Fixando $x^{*}=x_{0}+I$ e usando a igualdade (2.14) e a mudança de variáveis

$$
u=(x, t)=y(x+c t, t) \text { e } w(x, t)=z(x+c t, t)
$$

que satisfazem (1.81)

$$
\begin{aligned}
\left|p(x, t)-\phi\left(x-x^{*}\right)\right| & =\left|y\left(x-I-\eta(t)+c^{*} t+I, t\right)-\phi\left(x-I-x_{0}\right)\right| \\
& \leq\left|y\left(x-I-\eta(t)+c^{*} t+I, t\right)-y(x-I, t)\right|+\left|y(x-I, t)-\phi\left(x-I-x_{0}\right)\right| \\
& \leq \sup _{x \in \mathbb{R}}\left(\left|y_{x}(x, t)\right|\right)\left|-\eta(t)+c^{*} t+I\right|+\left|y(x-I, t)-\phi\left(x-I-x_{0}\right)\right| \\
& \leq C_{2} e^{-\bar{\omega} t} .
\end{aligned}
$$

E também

$$
\begin{aligned}
\left|q(x, t)-\psi\left(x-x^{*}\right)\right| & =\left|z\left(x-I-\eta(t)+c^{*} t+I, t\right)-\psi\left(x-I-x_{0}\right)\right| \\
& \leq\left|z\left(x-I-\eta(t)+c^{*} t+I, t\right)-z(x-I, t)\right|+\left|z(x-I, t)-\psi\left(x-I-x_{0}\right)\right| \\
& \leq \sup _{x \in \mathbb{R}}\left(\left|z_{x}(x, t)\right|\right)\left|-\eta(t)+c^{*} t+I\right|+\left|z(x-I, t)-\psi\left(x-I-x_{0}\right)\right| \\
& \leq C_{2} e^{-\bar{\omega} t} .
\end{aligned}
$$

Portanto, pelo Teorema 1.3.3 existe uma constante $C_{2}>0$ tal que

$$
\left|p(x, t)-\phi\left(x-x^{*}\right)\right| \leq C_{2} e^{-\bar{\omega} t}
$$

$\mathrm{e}$

$$
\left|q(x, t)-\psi\left(x-x^{*}\right)\right| \leq C_{2} e^{-\bar{\omega} t} .
$$




\section{Capítulo 3}

\section{Problema local em um intervalo limitado}

Para implementações numéricas é necessário limitar o domínio, por esta razão vamos estudar a existência e as propriedades espectrais de soluções estacionárias do problema não local em um domínio limitado. Muitas propriedades do problema não local são herdadas do problema local e por isso serão estudadas neste capítulo.

\subsection{Existência e unicidade de soluções estacionárias}

Vamos estudar a existência e unicidade de soluções estacionárias do problema local, em um intervalo $J:=\left[x_{-}, x_{+}\right] \subset \mathbb{R}$ tal que $0 \in J$ e $|J|=x_{+}-x_{-}$, dado por

$$
\left\{\begin{array}{l}
u_{t}=u_{x x}-c u_{x}+f(u)-w, \quad x \in J, \quad t>0 \\
w_{t}=-c w_{x}+\varepsilon(u-\gamma w),
\end{array}\right.
$$

com as condições iniciais $u(x, 0)=u_{0}(x)$ e $w(x, 0)=w_{0}(x)$. Ao problema (3.1) adicionamos as condições de contorno

$$
u\left(x_{-}, t\right)=w\left(x_{-}, t\right)=0 \text { e } u\left(x_{+}, t\right)=u_{2} .
$$

Como vimos na introdução, equilíbrios $(u, w)$ da equação (3.1) são soluções de

$$
\left\{\begin{aligned}
\phi^{\prime \prime}-c \phi^{\prime}+f(\phi)-\psi & =0 \\
-c \psi^{\prime}+\varepsilon(\phi-\gamma \psi) & =0
\end{aligned}\right.
$$

que devem satisfazer as condições: $\phi\left(x_{-}\right)=\psi\left(x_{-}\right)=0$ e $\phi\left(x_{+}\right)=u_{2}$.

Para encontrar soluções estacionárias do problema acima usaremos a mesma estratégia do Capítulo 1, isto é, encontrar soluções do problema equivalente

$$
\left\{\begin{array}{l}
u^{\prime}=v \\
v^{\prime}=c v-f(u)+w \\
w^{\prime}=\frac{\varepsilon}{c}(u-\gamma w)
\end{array}\right.
$$

com as condições

$$
\left(u\left(x_{-}\right), v\left(x_{-}\right), w\left(x_{-}\right)\right)=\left(0, v_{-}, 0\right) \quad \text { e } \quad\left(u\left(x_{+}\right), v\left(x_{+}\right), w\left(x_{+}\right)\right)=\left(u_{2}, v_{+}, w_{+}\right) \quad v_{ \pm}, w_{+}>0 .
$$

Sobre o problema (3.3) podemos identificar os seguintes aspectos sobre suas soluções:

i) Analisando o campo de vetores no ponto $\left(0, v_{-}, 0\right)$ concluímos que as soluções que iniciam 
neste ponto entram na região onde $u>0$ e $v>0$.

ii) Resolvendo a última equação de (3.4) com a condição $w\left(x_{-}\right)=0$, obtemos

$$
w(\xi)=\frac{\varepsilon}{c} \int_{x_{-}}^{\xi} e^{-\frac{\varepsilon \gamma}{c}(\xi-s)} u(s) d s
$$

e portanto $w>0$ enquanto $u>0$. Além disso usando a integração por partes na igualdade acima concluímos que

$$
w(\xi)=\frac{u(\xi)}{\gamma}-\frac{1}{\gamma} \int_{x_{-}}^{\xi} e^{\frac{\varepsilon \gamma}{c}(s-\xi)} u^{\prime}(s) d s
$$

Logo $w(\xi) \leq \frac{u(\xi)}{\gamma}$ enquanto $u^{\prime}(\xi)>0$.

Enquanto $u^{\prime}>0$, podemos expressar $v(\xi)$ e $w(\xi)$ como funções de $u(\xi)$, isto é, considere funções $m, n$ tais que $m(u(\xi))=v(\xi)$ e $n(u(\xi))=w(\xi)$. Pelo Teorema da Função Inversa, $m$ e $n$ satisfazem a EDO

$$
\left\{\begin{aligned}
\frac{d m}{d u} & =c-\frac{f(u)-n(u)}{m(u)} \\
\frac{d n}{d u} & =\frac{\varepsilon}{c} \frac{(u-\gamma n(u))}{m(u)}
\end{aligned}\right.
$$

com condições iniciais $m(0)=v_{-}$e $n(0)=0$. Portanto soluções de (3.4)-(3.5) podem ser vistas como uma curva no $\mathbb{R}^{3}$ cuja parametrização é dada por $u \longmapsto \Gamma:=(u, m(u), n(u))$.

Lema 3.1.1 Existe $c>0$ suficientemente pequeno tal que $\Gamma$ intercepta o plano $v=0$.

Demonstração. Suponha que para $c>0$ suficientemente pequeno não exista $\xi_{0} \in J$ tal que $v\left(\xi_{0}\right)=0$. Seja $\left\{c_{i}\right\}_{i \in \mathbb{N}}$ uma sequência tal que $\lim _{i \rightarrow \infty} c_{i}=0$.

Para cada $c_{i}$ considere $m_{i}$ e $n_{i}$ soluções de

$$
\left\{\begin{array}{l}
m_{i}^{\prime}=\frac{c_{i} m_{i}-f(u)+n_{i}}{m_{i}} \\
n_{i}^{\prime}=\frac{\varepsilon\left(u-\gamma n_{i}\right)}{c_{i} m_{i}} \\
m_{i}(0)=v_{-} \text {e } \quad n_{i}(0)=0 .
\end{array}\right.
$$

Pela primeira equação, obtemos

$$
\begin{aligned}
m_{i}^{\prime} m_{i} & =c_{i} m_{i}-f(u)+n_{i} \\
\frac{d}{d u}\left[\frac{\left(m_{i}\right)^{2}}{2}\right] & =c_{i} m_{i}-f(u)+n_{i} \\
\frac{\left(m_{i}(u)\right)^{2}}{2} & =\int_{0}^{u}\left(c_{i} m_{i}(s)-f(s)+n_{i}(s)\right) d s .
\end{aligned}
$$

Já pela segunda equação temos

$$
\begin{aligned}
n_{i}^{\prime}+\frac{\varepsilon \gamma}{c_{i} m_{i}} n_{i} & =\frac{\varepsilon u}{c_{i} m_{i}} \\
n_{i}(u) & =\frac{1}{\mu(u)} \int_{0}^{u} \frac{\varepsilon s}{c_{i} m_{i}(s)} \mu(s) d s
\end{aligned}
$$


onde $\mu(u)=\exp \left(\int_{0}^{u} \frac{\varepsilon \gamma}{c_{i} m_{i}(\tau)} d \tau\right)$.

Usando integração por partes, temos

$$
\begin{gathered}
n_{i}(u)=\frac{1}{\mu(u)}\left[\int_{0}^{u} s \frac{d \mu}{\gamma}\right] \\
n_{i}(u)=\frac{1}{\mu(u)}\left[\frac{u}{\gamma} \mu(u)-\frac{1}{\gamma} \int_{0}^{u} \mu(s) d s\right] \\
n_{i}(u)=\frac{u}{\gamma}-\frac{1}{\gamma} \int_{0}^{u} \exp \left(-\int_{s}^{u} \frac{\varepsilon \gamma}{c_{i} m_{i}(u)} d \tau\right) d s .
\end{gathered}
$$

Como $\lim _{i \rightarrow \infty} c_{i}=0$ e, se $0 \leq u \leq u_{2}$, então $\lim _{i \rightarrow \infty} n_{i}(u)=\frac{u}{\gamma}$. Desta forma, se $0 \leq u \leq u_{2}$ então

$$
\begin{aligned}
0<\lim _{i \rightarrow \infty} \frac{\left(m_{i}(u)\right)^{2}}{2} & =\lim _{i \rightarrow \infty} \int_{0}^{u_{2}}\left(c_{i} m_{i}(s)-f(s)+n_{i}(s)\right) d s \\
0 & <\int_{0}^{u_{2}}\left(-f(s)+\frac{s}{\gamma}\right) d s,
\end{aligned}
$$

o que contradiz a hipótese (H4). Logo, existe $c>0$ suficientemente pequeno tal que $\Gamma$ intercepta o plano $v=0$.

Observação: Seja $\mathcal{C}=\{c>0 ; \quad v(\xi)=0$, para algum $\xi \in J\}$. O conjunto $\mathcal{C}$ é um conjunto aberto, não vazio e limitado superiormente, pois se

$$
c>\sup _{u \in\left[0, u_{2}\right]}\left|\frac{f(u)-n(u)}{m(u)}\right|,
$$

então $m^{\prime}>0$ e, portanto, $v(\xi)>0$ para todo $\xi \in J$.

Definindo $c_{1}=\sup \mathcal{C}$ e pela discussão acima sobre as caraterísticas das soluções de (3.4) concluímos o resultado abaixo.

Corolário 3.1.1 Existe $c_{1}>0$ tal que para todo $c>c_{1}$ as soluçôes de (3.4)-(3.5) tem as seguintes propriedades

$$
0 \leq u(\xi) \leq u_{2}, \quad 0 \leq w(\xi) \leq \frac{u_{2}}{\gamma} \text { e } v(\xi)>0, \quad \text { para todo } \quad \xi \in J
$$

Para simplificar as demonstrações, a seguir vamos chamar de "soluções simples" toda solução $(u(\xi), v(\xi), w(\xi))$ de (3.4) que satisfaz as condições em (3.5).

Lema 3.1.2 Para cada $c>c_{1}$, temos os seguintes resultados:

(i) Se $\Gamma$ é uma solução simples, o tempo que ela leva do ponto $\left(u\left(\xi_{0}\right), v\left(\xi_{0}\right), w\left(\xi_{0}\right)\right)$ ao ponto $\left(u\left(\xi_{1}\right), v\left(\xi_{1}\right), w\left(\xi_{1}\right)\right)$, é dado por

$$
\xi_{1}-\xi_{0}=\int_{u\left(\xi_{0}\right)}^{u\left(\xi_{1}\right)} \frac{d u}{m(u)}
$$

Em particular, o tempo que leva do ponto $\left(0, v_{-}, 0\right)$ ao ponto $\left(u_{2}, v_{+}, w_{+}\right)$é dado por

$$
x_{+}-x_{-}=\int_{0}^{u_{2}} \frac{d u}{m(u)} .
$$

(ii) Existe um $c_{2}>0$ tal que, se $c>c_{2}$ então $\Gamma$ chega a um ponto $\left(u_{2}, v_{+}, w_{+}\right)$tal que $v_{+}>v_{-} e$, se $c<c_{2}$, $\Gamma$ chega a um ponto $\left(u_{2}, v_{+}, w_{+}\right)$tal que $v_{+}<v_{-}$. 
Demonstração. $(i)$ Como $v(\xi)=m(u(\xi))=\frac{d}{d \xi} u(\xi)$, então $d \xi=\frac{d u(\xi)}{m(u(\xi))}$. Logo,

$$
\xi_{1}-\xi_{0}=\int_{u\left(\xi_{0}\right)}^{u\left(\xi_{1}\right)} \frac{d u}{m(u)} .
$$

(ii) Considere o vetor normal $\vec{n}=(0,1,0)$ ao plano $v=v_{-}$e o campo de vetores das soluções de (3.4) no ponto $\left(u, v_{-}, w\right)$

$$
\mathrm{X}(u, v, w)=\left(v, c v-f(u)+w, \frac{\varepsilon}{c}(u-\gamma w)\right) .
$$

Logo

$$
\vec{n} \cdot \mathrm{X}(u, v, w)=c v_{-}-f(u)+w .
$$

Seja

$$
c_{2}>\sup _{0 \leq u \leq u_{2}} \frac{|f(u)-n(u)|}{v_{-}} .
$$

Se $c>c_{2}$, o produto escalar é positivo e, portanto, as soluções permanecem acima do plano $v=v_{-}$e $v^{\prime}(\xi)>0, \forall \xi \in J$, e portanto chegam a um certo valor $v_{+}>v_{-}$. Se $c<c_{2}$, $\Gamma$ "fura" o plano $v=v_{-}$e $v^{\prime}(\xi)<0$, para algum $\xi>x_{-}$, e portanto chega a um ponto $\left(u_{2}, v_{+}, w_{+}\right)$onde $v_{+}<v_{-}$.

Diante do exposto acima, podemos concluir o seguinte teorema:

Teorema 3.1.1 Para cada $c>c_{1}$, existe uma única solução $(\phi, \psi)$ de (3.3). Além disso, $\phi^{\prime}(\xi)>0$, para todo $\xi \in J$.

Demonstração. Pelo Corolário 3.1.1, se $c>c_{1}$, então $u^{\prime}(\xi)>0, \forall \xi \in J$, e portanto todas as soluções de (3.4) saindo do ponto $\left(0, v_{-}, 0\right)$ são soluções simples e o tempo que esta solução leva de $\left(0, v_{-}, 0\right)$ a $\left(u_{2}, v_{+}, w_{+}\right)$é dado por $x_{+}-x_{-}=\int_{0}^{u_{2}} \frac{d u}{m(u)}$. A unicidade segue por teoremas clássicos de existência e unicidade de sistemas de EDO. Veja, por exemplo, Sotomayor (1979).

\subsection{Análise espectral do problema em intervalo finito}

A análise espectral de sistemas parabólico-hiperbólico em intervalo finito foi estudada por Rottmann-Matthes (2005) como uma extensão do trabalho de Beyn e Lorenz (1999) feito para problemas parabólicos. Nesta primeira subseção, vamos, de forma sucinta, apresentar as hipóteses e os principais resultados da Tese de Rottmann-Matthes (2005) e depois verificar que estes resultados são aplicados ao nosso problema.

\subsubsection{Hipóteses e principais resultados}

Vamos considerar um sistema parabólico-hiperbólico da forma

$$
\left(\begin{array}{c}
u_{t} \\
w_{t}
\end{array}\right)=L\left(\begin{array}{c}
u \\
w
\end{array}\right):=\left(\begin{array}{cc}
A & 0 \\
0 & 0
\end{array}\right)\left(\begin{array}{l}
u_{x x} \\
w_{x x}
\end{array}\right)+\left(\begin{array}{cc}
B_{11} & B_{12} \\
0 & B_{22}
\end{array}\right)\left(\begin{array}{l}
u_{x} \\
w_{x}
\end{array}\right)+\left(\begin{array}{ll}
C_{11} & C_{12} \\
C_{21} & C_{22}
\end{array}\right)\left(\begin{array}{c}
u \\
w
\end{array}\right),
$$

onde $L: H^{2}\left(\mathbb{R}, \mathbb{C}^{n}\right) \times H^{1}\left(\mathbb{R}, \mathbb{C}^{m}\right) \rightarrow L^{2}\left(\mathbb{R}, \mathbb{C}^{n}\right) \times L^{2}\left(\mathbb{R}, \mathbb{C}^{m}\right)$.

Hipóteses sobre o operador $L$ 
i) As matrizes $B_{i j}(x)$ e $C_{i j}(x)$ são de classe $\mathcal{C}^{2}$ e satisfazem:

$$
\begin{gathered}
\lim _{x \rightarrow \pm \infty} B_{i j}(x):=B_{i j \pm} \text { e } \lim _{x \rightarrow \pm \infty} C_{i j}(x):=C_{i j \pm} \\
\lim _{x \rightarrow \pm \infty} \partial_{x} B_{22}(x)=0 ; \\
\left\|\partial_{x}^{2} B_{22}\right\|_{\infty}<\infty, \quad\left\|\partial_{x} C_{22}\right\|_{\infty}<\infty \text { e }\left\|\partial_{x} B_{12}\right\|_{\infty}<\infty .
\end{gathered}
$$

ii) A matriz $A \in \mathbb{C}^{n, n}$ satisfaz $A+A^{*} \geq \alpha I$, para algum $\alpha \in \mathbb{R}$.

iii) A função real $B_{22}$ é uma matriz diagonal e existem $b_{0}$ e $\beta>0$ tal que, para todo $x \in \mathbb{R}$, os elementos da diagonal satisfazem:

$$
\left|b_{i i}(x)-b_{j j}(x)\right| \geq \beta, \text { para } i \neq j,
$$

$b_{i i}(x) \geq b_{0}, \quad$ para $1 \leq i \leq r \quad$ e $\quad-b_{i i}(x) \geq b_{0}$, para $r+1 \leq i \leq m$.

Além disso, se $c_{22 \pm}$ são os elementos da diagonal da matriz $C_{22 \pm}$, então $R e c_{22 \pm}<-2 \delta$, para $\operatorname{algum} \delta>0$.

iv) Existe $\delta>0$ tal que, para todo $\tau \in \mathbb{R}$ e para todo $s \in \mathbb{C}$, a igualdade

$$
\operatorname{det}\left(-\tau^{2}\left(\begin{array}{cc}
A & 0 \\
0 & 0
\end{array}\right)+i \tau\left(\begin{array}{cc}
B_{11+} & B_{12+} \\
0 & B_{22+}
\end{array}\right)+\left(\begin{array}{cc}
C_{11+} & C_{12+} \\
C_{21+} & C_{22+}
\end{array}\right)-\mu I_{n+m}\right)=0
$$

$\mathrm{Ou}$

$$
\operatorname{det}\left(-\tau^{2}\left(\begin{array}{cc}
A & 0 \\
0 & 0
\end{array}\right)+i \tau\left(\begin{array}{cc}
B_{11-} & B_{12-} \\
0 & B_{22-}
\end{array}\right)+\left(\begin{array}{ll}
C_{11-} & C_{12-} \\
C_{21-} & C_{22-}
\end{array}\right)-\mu I_{n+m}\right)=0
$$

implica que $\operatorname{Re} s \leq-\delta$.

Para análise das propriedades espectrais de $L$ considere a equação resolvente

$$
(\mu I-L)\left(\begin{array}{c}
u \\
w
\end{array}\right)=\left(\begin{array}{c}
f \\
g
\end{array}\right)
$$

que é equivalente ao sistema de primeira ordem:

$$
L(\mu) z:=z_{x}-M(x, \mu) z=\left(\begin{array}{c}
0 \\
-f+B_{12} B_{22}^{-1} g \\
-B_{22} g
\end{array}\right)
$$

onde $z:=\left(u, A u_{x}, w\right)$,

$$
M(\cdot, \mu)=\left(\begin{array}{ccc}
0 & A^{-1} & 0 \\
B_{12} B_{22}^{-1} C_{21}+\left(\mu I-C_{11}\right) & -B_{11} A^{-1} & -C_{12}-B_{12} B_{22}^{-1}\left(\mu I_{m}-C_{22}\right) \\
-B_{22}^{-1} C_{21} & 0 & B_{22}^{-1}\left(\mu I_{m}-C_{22}\right)
\end{array}\right)
$$

e $L(s): H^{2}\left(\mathbb{R}, \mathbb{C}^{n}\right) \times H^{1}\left(\mathbb{R}, \mathbb{C}^{n}\right) \times H^{1}\left(\mathbb{R}, \mathbb{C}^{m}\right) \rightarrow H^{1}\left(\mathbb{R}, \mathbb{C}^{n}\right) \times L^{2}\left(\mathbb{R}, \mathbb{C}^{n}\right) \times L^{2}\left(\mathbb{R}, \mathbb{C}^{m}\right)$. Pela hipótese (iv), as matrizes $\lim _{x \rightarrow \pm \infty} M(x, \mu):=M_{ \pm}(\mu)$ existem e são hiperbólicas, para todo $\mu \in \mathbb{C}$ com $\operatorname{Re} \mu>$ $-\delta$ e, para nossos propósitos, iremos estudar apenas a parte do espectro que está contido no conjunto $\{\mu \in \mathbb{C} ; \operatorname{Re} \mu>-\delta\}$.

Denotamos por $V_{ \pm}^{u}(\mu) \subset \mathbb{C}^{l, r}$ a base do subespaço instável de $M_{ \pm}(\mu)$ e $V_{ \pm}^{s}(\mu) \subset \mathbb{C}^{l, p}$ a base do subespaço estável de $M_{ \pm}(\mu)$ tal que $p+r=l$.

Lema 3.2.1 $L(\mu)$ e $(\mu I-L)$ são operadores de Fredholm de indice zero para Re $\mu>-\delta$.

A proposta de Rottmann-Matthes (2005) consiste em aproximar o espectro de $L$ pelo espectro do operador $\left.L\right|_{J}$, o operador $L$ restrito a um intervalo limitado $J$. 
Considere o intervalo limitado com as seguintes características: $J=\left[x_{-}, x_{+}\right] \subset \mathbb{R} ; \quad 0 \in J$ e $|J|>1$ e a restrição da equação resolvente (3.6) a $J$

$$
\left(\mu I-\left.L\right|_{J}\right)\left(\begin{array}{c}
\left.u\right|_{J} \\
\left.w\right|_{J}
\end{array}\right)=\left(\begin{array}{c}
\left.f\right|_{J} \\
\left.g\right|_{J}
\end{array}\right) \quad \text { em } L^{2}\left(J, \mathbb{C}^{m}\right) \times L^{2}\left(J, \mathbb{C}^{n}\right),
$$

onde $\left.L\right|_{J}$ é definido da mesma forma que $L$, porém em um intervalo limitado $J$.

Para obter um problema bem posto no intervalo finito $J$ impomos as condições de contorno

$$
R\left(\begin{array}{c}
\left.u\right|_{J} \\
\left.w\right|_{J}
\end{array}\right):=R_{-}\left(\begin{array}{c}
\left.u\right|_{J}\left(x_{-}\right) \\
\left.u_{x}\right|_{J}\left(x_{-}\right) \\
\left.w\right|_{J}\left(x_{-}\right)
\end{array}\right)+R_{+}\left(\begin{array}{c}
\left.u\right|_{J}\left(x_{+}\right) \\
\left.u_{x}\right|_{J}\left(x_{+}\right) \\
\left.w\right|_{J}\left(x_{+}\right)
\end{array}\right) .
$$

onde $R_{-}, R_{+}$são elementos de $\mathbb{C}^{l, l}$

A hipótese crucial sobre $R$ é que

$$
D(\mu):=\operatorname{det}\left[R_{-} V_{-}^{s}(\mu), \quad R_{+} V_{+}^{u}(\mu)\right] .
$$

A condição acima estabelece que o subespaço estável e instável das soluções podem ser controlados pelos pontos extremos do intervalo.

Considere os espaços de Banach

$$
\begin{gathered}
E:=H^{2}\left(\mathbb{R}, \mathbb{C}^{n}\right) \times H^{1}\left(\mathbb{R}, \mathbb{C}^{m}\right), \quad F:=L^{2}\left(\mathbb{R}, \mathbb{C}^{n}\right) \times L^{2}\left(\mathbb{R}, \mathbb{C}^{m}\right) ; \\
\left.E\right|_{J}:=H^{2}\left(J, \mathbb{C}^{n}\right) \times H^{1}\left(J, \mathbb{C}^{m}\right),\left.\quad F\right|_{J}:=L^{2}\left(J, \mathbb{C}^{n}\right) \times L^{2}\left(J, \mathbb{C}^{m}\right) \times \mathbb{C}^{2 n+m} ;
\end{gathered}
$$

e os operadores

$\mathcal{A}(\mu):=\mu I-L \in L(E, F)$ e $\left.\mathcal{A}\right|_{J}(\mu):=\left(\begin{array}{c}\left.(\mu I-L)\right|_{J} \\ R\end{array}\right) \in L\left(\left.E\right|_{J},\left.F\right|_{J}\right)$.

Qualquer elemento não nulo do núcleo de $\mathcal{A}\left(\mu_{0}\right)$ é chamado de autoelemento de $\mathcal{A}(\cdot)$ associado ao autovalor $\mu_{0}$

Para um elemento $\mu_{0}$ no espectro normal $\sigma_{n}(L)$ de $L$, escolha $\varepsilon>0$ tal que $\mu_{0}$ é o único elemento do espectro de $L$ em uma bola fechada $\overline{B_{\varepsilon}\left(\mu_{0}\right)}$.

Vamos definir por $\mu_{0}$-grupo de autovalores de $\left.\mathcal{A}\right|_{J}$ o conjunto:

$$
\sigma_{J}:=\left\{\mu \in \overline{B_{\varepsilon}\left(\mu_{0}\right)}: \mu \text { é um autovalor de }\left.\mathcal{A}\right|_{J}(\cdot)\right\} .
$$

Teorema 3.2.1 Seja $\Sigma$ uma vizinhança aberta do autovalor isolado $\mu_{0}$ com $D(\mu) \neq 0$, para todo $\mu \in \Sigma$, e assuma que $\varepsilon$ é tão pequeno que $\overline{B_{\varepsilon}\left(\mu_{0}\right)} \subset \Sigma$. Então existe um intervalo compacto $J_{0} \subset \mathbb{R}$ tal que para todo intervalo compacto $J=\left[x_{-}, x_{+}\right]$com $J_{0} \subset J$ o $\mu_{0}$-grupo de autovalores $\sigma_{J}$ converge para o autovalor $\mu_{0}$ no sentido que, para cada $0<\beta^{\prime}<\min \left\{\beta_{-}, \beta_{+}\right\}$existe, $k:=k\left(\beta^{\prime}\right)>0$ tal que

$$
\max _{\mu \in \sigma_{J}}\left|\mu-\mu_{0}\right|=\operatorname{dist}\left(\sigma_{J}, \mu_{0}\right) \leq k e^{-\frac{\beta^{\prime}}{\kappa}\left(\min \left\{x_{+},-x_{-}\right\}\right)},
$$

onde $\kappa$ é a ordem máxima ${ }^{1}$ dos autoelementos de $\mathcal{A}(\cdot)$ associado ao autovalor $\mu_{0}$.

Demonstração. Veja Rottmann-Matthes (2005), pág. 106.

No caso de autovalores simples considere o operador $R$ como uma função $C^{1}$ de $\mu$ tal que

$$
R: \Sigma \rightarrow L\left(E, \mathbb{C}^{3}\right)
$$

\footnotetext{
${ }^{1}$ Veja Definição C.6 em Rottmann-Matthes (2005) pág 141
} 
é dada por

$$
R(\mu)\left(\begin{array}{c}
u \\
w
\end{array}\right)=R_{-}(\mu)\left(\begin{array}{c}
u\left(x_{-}\right) \\
u_{x}\left(x_{-}\right) \\
w\left(x_{-}\right)
\end{array}\right)+R_{+}(\mu)\left(\begin{array}{c}
u\left(x_{+}\right) \\
u_{x}\left(x_{+}\right) \\
w\left(x_{+}\right)
\end{array}\right)
$$

e

$$
D(\mu)=\operatorname{det}\left[R_{-}(\mu) V_{-}^{s}(\mu), \quad R_{+}(\mu) V_{+}^{u}(\mu)\right] .
$$

Teorema 3.2.2 Seja $\mu_{0} \in \sigma(L) \cap\{\operatorname{Re} \mu>-\delta\}$ um autovalor simples de $\mathcal{A}(\cdot)=\mu I-L$. Seja $\left(\phi_{0}, \psi_{0}\right)$ um autoelemento não trivial de $\mathcal{A}$ para o autovalor $\mu_{0}$. Além disso, assuma que $D\left(\mu_{0}\right) \neq 0$. Então existe um intervalo compacto $J_{0}$ e $\delta_{0}>0$ tais que, para todo intervalo compacto $J_{0} \subset J$, existe exatamente um autovalor simples $\mu_{J}$, com $\left|\mu_{0}-\mu_{J}\right| \leq \delta_{0}$, da aproximação no intervalo finito

$$
\mathcal{A}_{J}(\cdot): \mu \longmapsto\left(\begin{array}{c}
\mu I-L_{J} \\
R(\mu)
\end{array}\right)
$$

Além disso, existe uma correspondente autofunção $\left(\begin{array}{c}u_{J} \\ w_{J}\end{array}\right) \in \tilde{E}_{J}:=H^{2}(J, \mathbb{C}) \times H^{1}(J, \mathbb{C})$ tal que as estimativas são verdadeiras

$$
\left|\mu_{J}-\mu_{0}\right|+\left\|\left(\begin{array}{c}
u_{J} \\
w_{J}
\end{array}\right)-\left(\begin{array}{c}
\left.u_{0}\right|_{J} \\
\left.w_{0}\right|_{J}
\end{array}\right)\right\|_{\tilde{E}_{J}} \leq C\left|R_{-}\left(\mu_{0}\right)\left(\begin{array}{c}
u_{0}\left(x_{-}\right) \\
u_{0, x}\left(x_{-}\right) \\
w_{0}\left(x_{-}\right)
\end{array}\right)+R_{+}\left(\mu_{0}\right)\left(\begin{array}{c}
u_{0}\left(x_{+}\right) \\
u_{0, x}\left(x_{+}\right) \\
w_{0}\left(x_{+}\right)
\end{array}\right)\right|
$$

com a constante $C$ independente de $J$.

Demonstração. Veja Rottmann-Matthes (2005), pág. 108.

\subsubsection{Aplicação no sistema de FitzHugh-Nagumo}

Considere o operador $L: H^{2}(\mathbb{R}, \mathbb{C}) \times H^{1}(\mathbb{R}, \mathbb{C}) \rightarrow L^{2}(\mathbb{R}, \mathbb{C}) \times L^{2}(\mathbb{R}, \mathbb{C})$ dado por

$$
\left(\begin{array}{l}
u_{t} \\
w_{t}
\end{array}\right)=L\left(\begin{array}{c}
u \\
w
\end{array}\right):=\left(\begin{array}{ll}
1 & 0 \\
0 & 0
\end{array}\right)\left(\begin{array}{l}
u_{x x} \\
w_{x x}
\end{array}\right)+\left(\begin{array}{cc}
-c & 0 \\
0 & -c
\end{array}\right)\left(\begin{array}{c}
u_{x} \\
w_{x}
\end{array}\right)+\left(\begin{array}{cc}
f^{\prime}(\phi) & -1 \\
\varepsilon & -\varepsilon \gamma
\end{array}\right)\left(\begin{array}{c}
u \\
w
\end{array}\right)
$$

As hipóteses $(i)$ - (iii) são facilmente verificadas e a hipótese $(i v)$ já foi discutida na demonstração do Teorema 1.2.3.

A equação resolvente (3.6) no nosso caso é equivalente ao sistema

$$
L(\mu) z:=z_{x}-M(x, \mu) z=\left(\begin{array}{c}
0 \\
h \\
-\frac{g}{c}
\end{array}\right)
$$

onde

$$
M(\cdot, \mu)=\left(\begin{array}{ccc}
0 & 1 & 0 \\
\mu-f^{\prime}(\phi) & c & 1 \\
\frac{\varepsilon}{c} & 0 & \frac{-(\varepsilon \gamma+\mu)}{c}
\end{array}\right)
$$

Pelo Lema 1.2.3 a matriz $M_{-}(\mu)$ tem três autovalores satisfazendo $\operatorname{Re} r_{1}>0>\operatorname{Re} r_{2}>\operatorname{Re} r_{3}$ com autovetores associados

$$
\rho_{i}(\mu)=\left(1, r_{i}, \frac{\varepsilon}{c r_{i}+\mu+\varepsilon \gamma}\right),
$$

e a matriz $M_{+}(\mu)$ tem três autovalores satisfazendo $\operatorname{Re}\left\{s_{1}\right\}>0>\operatorname{Re}\left\{s_{2}\right\}>\operatorname{Re}\left\{s_{3}\right\}$ com autovetores associados

$$
\kappa_{i}(\mu)=\left(1, s_{i}, \frac{\varepsilon}{c s_{i}+\mu+\varepsilon \gamma}\right) .
$$


Sejam $V_{+}^{u}(\mu)=\left\{\kappa_{1}\right\}$ a base do subespaço instável de $M_{+}(\mu), V_{-}^{s}(\mu)=\left\{\rho_{2}, \rho_{3}\right\}$ base do subespaço estável de $M_{-}(\mu)$ e o operador

$$
\begin{aligned}
R(\mu)\left(\begin{array}{c}
u \\
w
\end{array}\right) & =\left(\begin{array}{lll}
1 & 0 & 0 \\
0 & 0 & 0 \\
0 & 0 & 1
\end{array}\right)\left(\begin{array}{c}
u\left(x_{-}\right) \\
u_{x}\left(x_{-}\right) \\
w\left(x_{-}\right)
\end{array}\right)+\left(\begin{array}{lll}
0 & 0 & 0 \\
1 & 0 & 0 \\
0 & 0 & 0
\end{array}\right)\left(\begin{array}{c}
u\left(x_{+}\right) \\
u_{x}\left(x_{+}\right) \\
w\left(x_{+}\right)
\end{array}\right)=\left(\begin{array}{c}
u\left(x_{-}\right) \\
u\left(x_{+}\right) \\
w\left(x_{-}\right)
\end{array}\right) . \\
D(\mu) & =\operatorname{det}\left[R_{-} V_{-}^{s}(\mu), R_{+} V_{+}^{u}(\mu)\right]=\operatorname{det}\left[\begin{array}{ccc}
1 & 1 & 0 \\
\frac{\varepsilon}{c r_{2}+\mu+\varepsilon \gamma} & \frac{\varepsilon}{c r_{3}+\mu+\varepsilon \gamma} & 0
\end{array}\right] \\
& =\frac{\varepsilon}{c r_{2}+\mu+\varepsilon \gamma}-\frac{\varepsilon}{c r_{3}+\mu+\varepsilon \gamma} \neq 0,
\end{aligned}
$$

para qualquer $\mu$ tal que $R e \mu \geq-\delta$.

Como as hipóteses dos Teoremas 3.2.1 e 3.2.2 são satisfeitas para o problema (3.1)-(3.2), podemos enunciar o seguinte resultado

Teorema 3.2.3 Existe $\delta_{0}>0$ e um intervalo compacto $J_{0}$ tais que existe exatamente um autovalor $\mu_{J}$ de $L_{J}$ tal que $\left|\mu_{J}\right| \leq \delta_{0}$, ou seja,

$$
\sigma\left(L_{J}\right) \cap\{\mu \in \mathbb{C} ; \operatorname{Re} \mu>-\delta\} \subset\left\{\mu \in \mathbb{C} ;|\mu| \leq \delta_{0}\right\} .
$$

Pelos resultados numéricos que Rottmann-Matthes (2005) obteve sobre a estabilidade da onda viajante do tipo pulso em um intervalo limitado, podemos supor que para um intervalo $J$ suficientemente grande temos:

$$
\sigma\left(L_{J}\right) \subset\{\mu \in \mathbb{C} ; \operatorname{Re} \mu<-\delta\}, \quad \text { para algum } \quad \delta>0 .
$$




\section{Capítulo 4}

\section{Problema não local em um intervalo limitado}

\subsection{Soluções estacionárias do problema não local}

Vamos estudar o problema não local

$$
\left\{\begin{array}{l}
p_{t}=p_{x x}-\lambda(p, q) p_{x}+f(p)-q, \\
q_{t}=-\lambda(p, q) q_{x}+\varepsilon(p-\gamma q),
\end{array} \quad x \in J, \quad t>0\right.
$$

com as condições iniciais $p(x, 0)=u_{0}(x)$ e $q(x, 0)=w_{0}(x)$ e as condições de contorno $p\left(x_{-}, t\right)=0$, $p\left(x_{+}, t\right)=u_{2}$ e $q\left(x_{-}, t\right)=0$, onde $J=\left[x_{-}, x_{+}\right] \subset \mathbb{R}, 0 \in J$ e $|J|=x_{+}-x_{-}$.

Além disso considere $\lambda(p, q)=\frac{A-\left\langle q, p_{x}\right\rangle_{L^{2}(J)}}{\left\|p_{x}\right\|_{L^{2}(J)}^{2}}, \operatorname{com} A=\int_{0}^{u_{2}} f(s) d s$

Lema 4.1.1 Considere o par $(\phi, \psi) \in H^{2}(J) \times H^{1}(J)$ tal que $\phi\left(x_{-}\right)=0, \phi\left(x_{+}\right)=u_{2}, \psi\left(x_{-}\right)=0$ $e$

$$
c=\lambda(\phi, \psi) \in \mathbb{R} .
$$

Então, $(\phi, \psi)$ é uma solução estacionária de (4.1) se, e somente se, $(\phi, \psi)$ é uma solução de (3.3) satisfazendo

$$
\phi^{\prime}\left(x_{-}\right)=\phi^{\prime}\left(x_{+}\right) .
$$

Demonstração. Se $(\phi, \psi)$ é uma solução estacionária de (4.1) $\operatorname{com} c=\lambda(\phi, \psi)$ então

$$
\left\{\begin{array}{l}
0=\phi_{x x}-c \phi_{x}+f(\phi)-\psi, \\
0=-c \psi_{x}+\varepsilon(\phi-\gamma \psi),
\end{array} \quad x \in J, \quad t>0 .\right.
$$

Logo $(\phi, \psi)$ é solução de (3.3).

Multiplicando a primeira equação de (4.4) por $\phi^{\prime}$ e integrando no intervalo $J$, obtemos

$$
\int_{x_{-}}^{x_{+}} \phi^{\prime \prime}(x) \phi^{\prime}(x) d x-\lambda(\phi, \psi) \int_{x_{-}}^{x_{+}} \phi^{\prime}(x)^{2} d x+A-\int_{x_{-}}^{x_{+}} \phi^{\prime}(x) \psi(x) d x=0,
$$

onde usamos o fato de que $\phi\left(x_{-}\right)=0, \phi\left(x_{+}\right)=u_{2}$ e $A=\int_{0}^{u_{2}} f(s) d s$.

A integração por partes nos permite concluir que

$$
\frac{1}{2}\left[\left(\phi^{\prime}\left(x_{+}\right)\right)^{2}-\left(\phi^{\prime}\left(x_{-}\right)\right)^{2}\right]-\lambda(\phi, \psi)\left\|\phi^{\prime}\right\|_{L^{2}(J)}^{2}+A-\left\langle\psi, \phi^{\prime}\right\rangle_{L^{2}(J)}=0 .
$$


Pela relação (4.2),

$$
\lambda(\phi, \psi)\left\|\phi^{\prime}\right\|_{L^{2}(J)}^{2}=A-\left\langle\psi, \phi^{\prime}\right\rangle_{L^{2}(J)},
$$

e, portanto, $\phi^{\prime}\left(x_{-}\right)=\phi^{\prime}\left(x_{+}\right)$.

Por outro lado, se $(\phi, \psi)$ é solução de (3.3) e satisfaz a condição $\phi^{\prime}\left(x_{-}\right)=\phi^{\prime}\left(x_{+}\right)$como mostramos no Capitulo 2, ela é uma solução da equação

$$
\left\{\begin{array}{c}
\phi^{\prime \prime}(x)-\frac{A-\left\langle\phi^{\prime}, \psi\right\rangle}{\left\langle\phi^{\prime}, \phi^{\prime}\right\rangle} \phi^{\prime}(x)+f(\phi(x))-\psi(x)=0 \\
-\frac{\varepsilon\left(B-\left\langle\phi^{\prime}, \psi\right\rangle\right)}{\left\langle\psi^{\prime}, \psi^{\prime}\right\rangle} \psi^{\prime}(x)+\varepsilon(\phi(x)-\gamma \psi(x))=0 .
\end{array}\right.
$$

Portanto um equilíbrio de (4.1).

Teorema 4.1.1 Existe uma única solução estacionária $(\phi, \psi)$ de (4.1).

Demonstração. Pelo Lema 4.1.1, uma solução estacionária $(\phi, \psi)$ de (4.1) é uma solução de (3.3) que satisfaz a condição $\phi^{\prime}\left(x_{-}\right)=\phi^{\prime}\left(x_{+}\right)$.

Pelo Teorema 3.1.1, para cada $c>c_{1}$, existe uma única solução $(\phi, \psi)$ de (3.3) que é equivalente as coordenadas $u$ e $w$ das soluções simples de (3.4). Dentre essas soluções, a que estamos procurando são aquelas cuja componente $v$ satisfaz $v\left(x_{-}\right)=v\left(x_{+}\right)$.

Seja $c_{2}$ do Lema 3.1.2, por dependência contínua em relação ao parâmetro $c$ das soluções simples $\Gamma$ de (3.4) existe $c_{J} \in\left(c_{2}-\delta, c_{2}+\delta\right)$, para algum $\delta>0$, tal que $\Gamma$ sai do ponto $\left(0, v_{-}, 0\right)$ e chega ao ponto $\left(u_{2}, v_{+}, w_{+}\right)$onde $v_{-}=v_{+}$. Logo, existe uma única solução $(\phi, \psi)$ de (3.3) com a propriedade $\phi^{\prime}\left(x_{-}\right)=\phi^{\prime}\left(x_{+}\right)$e é, portanto, a única solução estacionária de (4.1).

\subsubsection{Convergência das soluções estacionárias para a onda viajante quando $|J| \rightarrow \infty$}

Nesta seção vamos estudar o comportamento da solução estacionária encontrada no Teorema 4.1.1 em relação ao tamanho do intervalo $J$. Observe que, se $\left(\phi(\xi), \phi^{\prime}(\xi), \psi(\xi)\right)$ é uma solução de (3.4) então $\left(\phi(\xi+k), \phi^{\prime}(\xi+k), \psi(\xi+k)\right)$ também é uma solução, para qualquer constante $k \in \mathbb{R}$, logo podemos considerar as soluções de (3.4) no intervalo $[0, r]$ e analisar essas soluções quando $r \rightarrow \infty$.

Vamos denotar por $\left(\phi_{r}, \psi_{r}\right)$ a solução estacionária de (4.1) no intervalo $[0, r]$ e $\left(\phi_{\infty}, \psi_{\infty}\right)$ a onda viajante do problema original encontrada no Teorema 1.1.2.

Nos resultados a seguir considere

$$
\lambda^{r}:=\lambda\left(\phi_{r}, \psi_{r}\right) \quad \text { e } \quad \lambda^{\infty}:=\lambda\left(\phi_{\infty}, \psi_{\infty}\right) .
$$

Lema 4.1.2 Seja $\lambda^{r}$ tal que exista uma única solução de (4.1)-(4.3) no intervalo J. Então

$$
\left|\lambda^{r}-\lambda^{\infty}\right| \rightarrow 0, \quad \text { quando } r \rightarrow \infty,
$$

onde $\lambda^{\infty}=c^{*}$ do Teorema 1.1.2, isto é, a velocidade de propagação da onda viajante da equação $(1)$.

Demonstração. Considere o problema (3.4) no intervalo $[0, r]$ com as condições

$$
(u(0), v(0), w(0))=\left(0, v_{-}, 0\right) \quad \text { e } \quad(u(r), v(r), w(r))=\left(u_{2}, v_{+}, w_{+}\right), \quad v_{ \pm}, w_{+}>0 .
$$

Suponha que exista uma sequência $\left\{r_{n}\right\}_{n \in \mathbb{N}}$ tal que $r_{n} \rightarrow \infty$, quando $n \rightarrow \infty$, e $\delta>0$ tal que $\lambda^{r_{n}}>\lambda^{\infty}+\delta$ para todo $r_{n}$.

Da mesma forma como discutimos no Capítulo 3, podemos escrever $m(u)=v$ e $n(u)=w$ e ver as soluções de (3.4) como uma curva no $\mathbb{R}^{3}$ cuja parametrização é dada por $\Gamma:=(u, m(u), n(u))$. 
Para cada $r_{n}$, considere a curva $\Gamma_{r_{n}}:=\left(u, m_{r_{n}}(u), n_{r_{n}}(u)\right)$ que representa a solução do problema (3.4)-(4.6).

Pelo estudo do espaço de fase feito no Capítulo 1 , se $c>c^{*}+\varepsilon$ então $v(\xi)>0$, para todo $\xi>0$. Dessa forma, para cada $r_{n}$ existe $\beta>0$, tal que $m_{r_{n}}(u) \geq \beta$ para todo $u \in\left[0, u_{2}\right]$.

Mas, pelo Lema 3.1.2,

$$
r_{n}=\int_{0}^{u_{2}} \frac{d u}{m_{r_{n}}(u)} \leq \frac{u_{2}}{\beta}
$$

contradizendo o fato que $r_{n} \rightarrow \infty$.

Para o caso $\lambda^{r_{n}}<\lambda^{\infty}-\varepsilon$ sabemos que $v\left(\xi_{0}\right)=0$, para algum $\xi_{0}>0$, onde $u_{1} \leq u\left(\xi_{0}\right) \leq u_{2}$, ou seja, a solução entra na região

$$
T=\left\{(u, v, w) ; u_{2}<u<1, w<f(u), v<0\right\},
$$

o que contradiz a definição de $\lambda^{r_{n}}$ que definimos no Teorema 3.1.1.

Lema 4.1.3 Seja $\left(\phi_{r}, \phi_{r}^{\prime}, \psi_{r}\right)$ o equilibrio obtido no Teorema 4.1 .1 e $\left(\phi_{\infty}, \phi_{\infty}^{\prime}, \psi_{\infty}\right)$ a onda viajante encontrada no Teorema 1.1.2. Se $v_{-}>0$ for suficientemente pequeno, então

$$
\left.\left(\phi_{r}, \phi_{r}^{\prime}, \psi_{r}\right) \rightarrow\left(\phi_{\infty}, \phi_{\infty}^{\prime}, \psi_{\infty}\right)\right|_{J}, \quad \text { quando } \quad r \rightarrow \infty
$$

onde $\left.\left(\phi_{\infty}, \phi_{\infty}^{\prime}, \psi_{\infty}\right)\right|_{J}$ é a restrição de $\left(\phi_{\infty}, \phi_{\infty}^{\prime}, \psi_{\infty}\right)$ no intervalo $J=[0, r]$.

Demonstração. No Capítulo 1 vimos que $\left(\phi_{\infty}, \phi_{\infty}^{\prime}, \psi_{\infty}\right)$ pode ser vista como uma solução do problema (5)-(6) e pelos comentários feitos antes do Lema 1.1.3 podemos selecionar $(u(0), v(0), w(0))$ da curva integral $\Gamma=\left(\phi_{\infty}, \phi_{\infty}^{\prime}, \psi_{\infty}\right)$ suficientemente próximo de $(0,0,0)$, ou seja, podemos ver $\left.\left(\phi_{\infty}, \phi_{\infty}^{\prime}, \psi_{\infty}\right)\right|_{J}$ como solução do problema

$$
\left\{\begin{aligned}
u^{\prime} & =v \\
v^{\prime} & =c v-f(u)+w \\
w^{\prime} & =\frac{\varepsilon}{c}(u-\gamma w)
\end{aligned}\right.
$$

com condição inicial $(u(0), v(0), w(0))=\left(u^{0}, v^{0}, w^{0}\right)$, onde $\left|u^{0}\right|+\left|v^{0}\right|+\left|w^{0}\right|<\epsilon_{1}$, para algum $\epsilon_{1}>0$ suficientemente pequeno.

Seja $v_{-}>0$ suficientemente pequeno e $\left(\phi_{\infty}, \phi_{\infty}^{\prime}, \psi_{\infty}\right)$ uma solução de (4.7) com condição inicial $(u(0), v(0), w(0))=\left(0, v_{-}, 0\right)$. Pelo Teorema da Dependência Contínua em relação as condições iniciais: para todo $\epsilon_{1}>0$, existe $\delta>0$ tal que, se $\left|v_{-}-v^{0}\right|<\delta$, então

$$
\left|\phi_{\infty}\left(\xi ; v_{-}\right)-\phi_{\infty}\left(\xi ; v^{0}\right)\right|<\frac{\epsilon_{1}}{2}, \quad \xi \in J .
$$
$\delta$.

Como $\lim _{r \rightarrow \infty} \lambda^{r}=\lambda^{\infty}$, para todo $\delta>0$, existe $r_{0}>0$ tal que, para todo $r \geq r_{0}$, tem-se $\left|\lambda^{r}-\lambda^{\infty}\right|<$

Pelo Teorema da Dependência Contínua em relação aos parâmetros: para todo $\epsilon_{2}>0$, existe $\delta>0$ tal que, se $\left|\lambda^{r}-\lambda^{\infty}\right|<\delta$, então

$$
\left|\phi_{r}\left(\xi ; v_{-}\right)-\phi_{\infty}\left(\xi ; v_{-}\right)\right|<\frac{\epsilon_{2}}{2}, \quad \xi \in J .
$$

Logo, para todo $\epsilon>0$, existe $r_{0}>0$ tal que, $\forall r \geq r_{0}$,

$$
\left|\phi_{r}\left(\xi ; v_{-}\right)-\phi_{\infty}\left(\xi ; v^{0}\right)\right| \leq\left|\phi_{r}\left(\xi ; v_{-}\right)-\phi_{\infty}\left(\xi ; v_{-}\right)\right|+\left|\phi_{\infty}\left(\xi ; v_{-}\right)-\phi_{\infty}\left(\xi ; v^{0}\right)\right|<\epsilon, \quad \xi \in J .
$$

De forma análoga, provamos para as outras componentes. 
Vamos normalizar a órbita $\left(\phi_{r}, \phi_{r}^{\prime}, \psi_{r}\right)$ tal que $\phi_{r}(0)=a$ assim como já mencionamos no Capítulo 1 , onde normalizamos $\left(\phi_{\infty}, \phi_{\infty}^{\prime}, \psi_{\infty}\right)$ por $\phi_{\infty}(0)=a$.

\subsection{Propriedades espectrais}

Para analisar as propriedades espectrais do equilíbrio $\left(\phi_{r}, \psi_{r}\right)$ encontrado na seção anterior, vamos considerar a linearização de $(4.1)$ ao redor de $\left(\phi_{r}, \psi_{r}\right)$ dada por

$$
\left\{\begin{array}{l}
m_{t}=m_{x x}-\lambda\left(\phi_{r}, \psi_{r}\right) m_{x}+f^{\prime}\left(\phi_{r}\right) m-n+\phi_{r}^{\prime} \Pi^{r}(m, n), \\
n_{t}=-\lambda\left(\phi_{r}, \psi_{r}\right) n_{x}+\varepsilon(m-\gamma n)+\psi_{r}^{\prime} \Pi^{r}(m, n),
\end{array} \quad x \in J, \quad t>0\right.
$$

com as condições $m\left(x_{-}, t\right)=m\left(x_{+}, t\right)=0$ e $n\left(x_{-}, t\right)=0$.

O termo $\Pi^{r}(m, n)$ tem a forma

$$
\Pi^{r}(m, n)=\frac{2 \lambda^{r}\left\langle\phi_{r}^{\prime}, m_{x}\right\rangle+\left\langle\psi_{r}, m_{x}\right\rangle+\left\langle\phi_{r}^{\prime}, n\right\rangle}{\left\|\phi_{r}^{\prime}\right\|^{2}}
$$

e, tanto o produto interno, quanto a norma são consideradas em $L^{2}(J)$.

Considere o operador linear $L^{r}: D\left(L^{r}\right) \subset L^{2}(J) \times L^{2}(J) \rightarrow L^{2}(J) \times L^{2}(J)$, onde

$$
D\left(L^{r}\right)=\left\{(m, n) \in H^{2}(J) \times H^{1}(J) ; \quad m\left(x_{-}\right)=m\left(x_{+}\right)=0 \text { e } n\left(x_{-}\right)=0\right\},
$$

definido por

$$
L^{r}\left(\begin{array}{c}
m \\
n
\end{array}\right)=\left(\begin{array}{c}
m_{x x}-\lambda^{r} m_{x}+f^{\prime}\left(\phi_{r}\right) m-n+\phi_{r}^{\prime} \Pi^{r}(m, n) \\
-\lambda^{r} n_{x}+\varepsilon(m-\gamma n)+\psi_{r}^{\prime} \Pi^{r}(m, n)
\end{array}\right) .
$$

Considere o operador local no intervalo $J$

$$
L_{0}^{r}\left(\begin{array}{c}
m \\
n
\end{array}\right)=\left(\begin{array}{c}
m_{x x}-\lambda^{r} m_{x}+f^{\prime}\left(\phi_{r}\right) m-n \\
-\lambda^{r} n_{x}+\varepsilon(m-\gamma n)
\end{array}\right) .
$$

Podemos escrever

$$
L^{r}\left(\begin{array}{c}
m \\
n
\end{array}\right)=L_{0}^{r}\left(\begin{array}{c}
m \\
n
\end{array}\right)+\Pi^{r}(m, n)\left(\begin{array}{c}
\phi_{r}^{\prime} \\
\psi_{r}^{\prime}
\end{array}\right)
$$

\subsubsection{Propriedades espectrais de $L^{r}$}

Pela relação dada em (4.8) entre $L^{r}$ e $L_{0}^{r}$ temos que $\mu$ é um autovalor de $L_{0}^{r}$ se, e somente se, $\mu$ for um autovalor de $L^{r}$ de $\sigma\left(L^{r}\right)$ com o resolvente de $L_{0}^{r}, \rho\left(L_{0}^{r}\right)$.

Lema 4.2.1 Seja $\mu \in \sigma\left(L^{r}\right) \cap \rho\left(L_{0}^{r}\right)$. Então, $\mu$ é um autovalor geometricamente simples, isto é, $\operatorname{Ker}\left(L^{r}-\mu I\right)$ é unidimensional. Além disso, o autoespaço associado é gerado por $Y=\left(y_{1}, y_{2}\right)$, a única solução de

$$
\left(L_{0}^{r}-\mu I\right) Y=\left(\begin{array}{c}
\phi_{r}^{\prime} \\
\psi_{r}^{\prime}
\end{array}\right)
$$

$e$

$$
\Pi^{r}(Y)=-1
$$

Demonstração. Seja $Z=\left(z_{1}, z_{2}\right) \neq(0,0)$ tal que $L^{r} Z=\mu Z$, logo

$$
0=\left(L^{r}-\mu I\right) Z=\left(L_{0}^{r}-\mu I\right) Z+\Pi^{r}(Z)\left(\begin{array}{c}
\phi_{r}^{\prime} \\
\psi_{r}^{\prime}
\end{array}\right)
$$


Seja $Y$ tal que $\left(L_{0}^{r}-\mu I\right) Y=\left(\begin{array}{c}\phi_{r}^{\prime} \\ \psi_{r}^{\prime}\end{array}\right) \operatorname{logo}$

$$
\begin{aligned}
0=\left(L_{0}^{r}-\mu I\right) Z+\Pi^{r}(Z)\left(\begin{array}{c}
\phi_{r}^{\prime} \\
\psi_{r}^{\prime}
\end{array}\right) & =\left(L_{0}^{r}-\mu I\right) Z+\Pi^{r}(Z)\left(L_{0}^{r}-\mu I\right) Y \\
& =\left(L_{0}^{r}-\mu I\right)\left(Z+\Pi^{r}(Z) Y\right) .
\end{aligned}
$$

Como $\mu \in \rho\left(L_{0}^{r}\right)$ então $\left(L_{0}^{r}-\mu I\right)$ é injetora, e portanto, $Z=-\Pi^{r}(Z) Y$. Como $\Pi^{r}$ é um operador linear $\Pi^{r}(Y)=\Pi^{r}\left(\frac{-Z}{\Pi^{r}(Z)}\right)=-1$.

Pelo comentário no final do Capítulo 3, podemos supor que $\sigma\left(L_{0}^{r}\right) \subset\{s \in \mathbb{C}: \operatorname{Re} s<0\}$ e, com esta hipótese, temos o seguinte resultado:

Proposição 4.2.1 Nẫo existe autovalor real $\mu \geq 0$ no espectro de $L^{r}$.

Demonstração. Suponha que exista um autovalor $\mu \geq 0$ de $L^{r}$, logo $\mu \in \rho\left(L_{0}^{r}\right)$.

Para $\mu \geq 0$ e $\mu \in \sigma\left(L^{r}\right) \cap \rho\left(L_{0}^{r}\right)$ considere $(m, n)$ tal que

$$
\left(L_{0}^{r}-\mu I\right)\left(\begin{array}{c}
m \\
n
\end{array}\right)=\left(\begin{array}{c}
\phi_{r}^{\prime} \\
\psi_{r}^{\prime}
\end{array}\right)
$$

isto é,

$$
\left\{\begin{array}{l}
m^{\prime \prime}-\lambda^{r} m^{\prime}+\left(f^{\prime}\left(\phi_{r}\right)-\mu\right) m-n=\phi_{r}^{\prime} \\
-\lambda^{r} n^{\prime}+\varepsilon(m-\gamma n)=\psi_{r}^{\prime} .
\end{array}\right.
$$

Multiplicando a primeira equação de (4.9) por $\phi_{r}^{\prime}$ e integrando em $J$, obtemos

$$
\left\langle m^{\prime \prime}, \phi_{r}^{\prime}\right\rangle-\lambda^{r}\left\langle m^{\prime}, \phi_{r}^{\prime}\right\rangle+\left\langle f^{\prime}\left(\phi_{r}\right) m, \phi_{r}^{\prime}\right\rangle-\left\langle n, \phi_{r}^{\prime}\right\rangle-\mu\left\langle m, \phi_{r}^{\prime}\right\rangle=\left\|\phi_{r}^{\prime}\right\|^{2} .
$$

Usando integração por partes em $\left\langle f^{\prime}\left(\phi_{r}\right) m, \phi_{r}^{\prime}\right\rangle$ e $m\left(x_{ \pm}\right)=0$, obtemos

$$
\begin{aligned}
\left\langle f^{\prime}\left(\phi_{r}\right) m, \phi_{r}^{\prime}\right\rangle & =-\left\langle m^{\prime}, f\left(\phi_{r}\right)\right\rangle \\
& =\left\langle m^{\prime}, \phi_{r}^{\prime \prime}\right\rangle-\lambda^{r}\left\langle m^{\prime}, \phi_{r}^{\prime}\right\rangle+\left\langle m^{\prime}, \psi_{r}\right\rangle .
\end{aligned}
$$

Substituindo a igualdade acima na equação (4.10), temos

$$
\begin{aligned}
&\left\langle m^{\prime \prime}, \phi_{r}^{\prime}\right\rangle+\left\langle m^{\prime}, \phi_{r}^{\prime \prime}\right\rangle-2 \lambda^{r}\left\langle m^{\prime}, \phi_{r}^{\prime}\right\rangle-\left\langle n, \phi_{r}^{\prime}\right\rangle-\left\langle m^{\prime}, \psi_{r}\right\rangle-\mu\left\langle m, \phi_{r}^{\prime}\right\rangle=\left\|\phi_{r}^{\prime}\right\|^{2} \\
& \frac{\left\langle m^{\prime \prime}, \phi_{r}^{\prime}\right\rangle}{\left\|\phi_{r}^{\prime}\right\|^{2}}+\frac{\left\langle m^{\prime}, \phi_{r}^{\prime \prime}\right\rangle}{\left\|\phi_{r}^{\prime}\right\|^{2}}-\frac{2 \lambda^{r}\left\langle m^{\prime}, \phi_{r}^{\prime}\right\rangle+\left\langle n, \phi_{r}^{\prime}\right\rangle+\left\langle m^{\prime}, \psi_{r}\right\rangle}{\left\|\phi_{r}^{\prime}\right\|^{2}}-\mu \frac{\left\langle m, \phi_{r}^{\prime}\right\rangle}{\left\|\phi_{r}^{\prime}\right\|^{2}}=1 .
\end{aligned}
$$

Pelo Lema 4.2.1, $\Pi^{r}(m, n)=\frac{2 \lambda^{r}\left\langle m^{\prime}, \phi_{r}^{\prime}\right\rangle+\left\langle n, \phi_{r}^{\prime}\right\rangle+\left\langle m^{\prime}, \psi_{r}\right\rangle}{\left\|\phi_{r}^{\prime}\right\|^{2}}=-1$, logo

$$
\left\langle m^{\prime \prime}, \phi_{r}^{\prime}\right\rangle+\left\langle m^{\prime}, \phi_{r}^{\prime \prime}\right\rangle=\mu\left\langle m, \phi_{r}^{\prime}\right\rangle .
$$

Como $\left(\mu-L_{0}^{r}\right)\left(\begin{array}{c}m \\ n\end{array}\right)=-\left(\begin{array}{c}\phi_{r}^{\prime} \\ \psi_{r}^{\prime}\end{array}\right)$ e $\phi_{r}^{\prime}>0$, pelo princípio do máximo ${ }^{1}$, temos então $m(x)<$ 0 , para $x \in J$.

Por integração por partes

$$
\left\langle m^{\prime \prime}, \phi_{r}^{\prime}\right\rangle=\phi_{r}^{\prime}\left(x_{+}\right) m^{\prime}\left(x_{+}\right)-\phi_{r}^{\prime}\left(x_{-}\right) m^{\prime}\left(x_{-}\right)-\left\langle m^{\prime}, \phi_{r}^{\prime \prime}\right\rangle
$$

\footnotetext{
${ }^{1}$ veja por exemplo Protter e Weinberger (2012), pág. 190.
} 
Do Lema 4.1.1, seja $K=\phi_{r}^{\prime}\left(x_{-}\right)=\phi_{r}^{\prime}\left(x_{+}\right)$, então

$$
K\left[m^{\prime}\left(x_{+}\right)-m^{\prime}\left(x_{-}\right)\right]=\left\langle m^{\prime \prime}, \phi_{r}^{\prime}\right\rangle+\left\langle m^{\prime}, \phi_{r}^{\prime \prime}\right\rangle=\mu\left\langle m, \phi_{r}^{\prime}\right\rangle \leq 0
$$

e, portanto, $m^{\prime}\left(x_{+}\right) \leq m^{\prime}\left(x_{-}\right)$.

Se $m<0$ e $m\left(x_{-}\right)=0$ então $m^{\prime}\left(x_{-}\right) \leq 0$, caso contrário $m$ seria crescente e positiva em uma vizinhança de $x_{-}$.

Se $m<0$ e $m\left(x_{+}\right)=0$ então $m^{\prime}\left(x_{+}\right) \geq 0$, caso contrário $m$ seria decrescente e positiva na vizinhança de $x_{+}$.

Ou seja, $m$ deve ser decrescente na vizinhança de $x_{-}$e crescente na vizinhança de $x_{+}$logo $m^{\prime}\left(x_{-}\right) \leq 0 \leq m^{\prime}\left(x_{+}\right)$. Mas, acima, afirmamos que $m^{\prime}\left(x_{+}\right) \leq m^{\prime}\left(x_{-}\right)$, então só nos resta concluir que

$$
m^{\prime}\left(x_{+}\right)=m^{\prime}\left(x_{-}\right)=0 .
$$

Veja que $(m, n)$ satisfaz a equação (4.9) e as condições $m\left(x_{-}\right)=n\left(x_{-}\right)=0$. Se, além disso, $m^{\prime}\left(x_{-}\right)=0$ então

$$
m^{\prime \prime}\left(x_{-}\right)-\lambda^{r} m^{\prime}\left(x_{-}\right)+f^{\prime}\left(\phi\left(x_{-}\right)\right) m\left(x_{-}\right)-\mu m\left(x_{-}\right)-n\left(x_{-}\right)=\phi_{r}^{\prime}\left(x_{-}\right),
$$

ou seja, $m^{\prime \prime}\left(x_{-}\right)=\phi_{r}^{\prime}\left(x_{-}\right)>0$ e, portanto, $m$ seria positiva para uma vizinhança de $x_{-}$, o que é um absurdo.

\subsubsection{Propriedades espectrais de $L^{\infty}$}

Seja o operador não local $L^{\infty}: H^{2}(\mathbb{R}, \mathbb{C}) \times H^{1}(\mathbb{R}, \mathbb{C}) \rightarrow L^{2}(\mathbb{R}, \mathbb{C})$

$$
L^{\infty}\left(\begin{array}{c}
m \\
n
\end{array}\right)=\left(\begin{array}{l}
m_{x x}-\lambda^{\infty} m_{x}+f^{\prime}\left(\phi_{\infty}\right) m-n+\phi_{\infty}^{\prime} \Pi^{\infty}(m, n) \\
-\lambda^{\infty} n_{x}+\varepsilon(m-\gamma n)+\psi_{\infty}^{\prime} \Pi^{\infty}(m, n)
\end{array}\right),
$$

que pode ser reescrito na forma

$$
L^{\infty}\left(\begin{array}{c}
m \\
n
\end{array}\right)=L_{0}^{\infty}\left(\begin{array}{c}
m \\
n
\end{array}\right)+\Pi^{\infty}(m, n)\left(\begin{array}{c}
\phi_{\infty}^{\prime} \\
\psi_{\infty}^{\prime}
\end{array}\right)
$$

onde $L_{0}^{\infty}$ é o operador local em $\mathbb{R}$

$$
L_{0}^{\infty}\left(\begin{array}{c}
m \\
n
\end{array}\right)=\left(\begin{array}{l}
m_{x x}-\lambda^{\infty} m_{x}+f^{\prime}\left(\phi_{\infty}\right) m-n \\
-\lambda^{\infty} n_{x}+\varepsilon(m-\gamma n)
\end{array}\right)
$$

e

$$
\Pi^{\infty}(m, n)=\frac{2 \lambda^{\infty}\left\langle\phi_{\infty}^{\prime}, m_{x}\right\rangle+\left\langle\psi_{\infty}, m_{x}\right\rangle+\left\langle\phi_{\infty}^{\prime}, n\right\rangle}{\left\|\phi_{\infty}^{\prime}\right\|^{2}} .
$$

Lema 4.2.2 Considere o operador (4.13) e seu espectro $\sigma\left(L_{0}^{\infty}\right)$, são verdadeiras as afirmaçôes:

i) Existe $\delta>0$ tal que $\sigma\left(L_{0}^{\infty}\right) \backslash\{0\} \subset\{\mu \in \mathbb{C} ; \operatorname{Re} \mu \leq-\delta\}$;

ii) 0 é um autovalor simples de $L_{0}^{\infty}$ com autofunção associada $\left(\phi^{\prime}, \psi^{\prime}\right)$, isto é, não existe $(\bar{u}, \bar{w}) \in$ $H^{2}(\mathbb{R}) \times H^{1}(\mathbb{R})$ tal que

$$
L_{0}^{\infty}\left(\begin{array}{c}
\bar{u} \\
\bar{w}
\end{array}\right)=\left(\begin{array}{c}
\phi^{\prime} \\
\psi^{\prime}
\end{array}\right)
$$

Demonstração. Como $\lambda^{\infty}=c^{*}$, este é o mesmo resultado provado no Teorema 1.3.3. 
Lema 4.2.3 O operador $(m, n) \longmapsto \Pi^{\infty}(m, n)\left(\begin{array}{c}\phi_{\infty}^{\prime} \\ \psi_{\infty}^{\prime}\end{array}\right)$ é compacto e tem posto 1 .

Demonstração. Seja $F(m, n):=\Pi^{\infty}(m, n) \Phi_{\infty}$ onde $\Phi_{\infty}=\left(\begin{array}{c}\phi_{\infty}^{\prime} \\ \psi_{\infty}^{\prime}\end{array}\right)$ é fixo. Pela definição de $\Pi^{\infty}$, $F$ é um operador linear contínuo e, portanto, existe $C>0$ tal que

$$
\|F\| \leq C\left\|\Phi_{\infty}\right\| .
$$

Seja $K \subset D(F)$ um conjunto limitado. Então

$$
F(K)=\left\{\Pi^{\infty}(X) \Phi_{\infty}: X \in K\right\} \quad \subset \operatorname{span}\left\{\Phi_{\infty}\right\} .
$$

Se $Y \in F(K)$ então $\exists X \in K$ tal que $Y=\Pi^{\infty}(X) \Phi_{\infty}$ e, portanto,

$$
\|Y\| \leq C\left\|\Phi_{\infty}\right\|<\infty
$$

Como $F(K)$ é limitado e está contido em um espaço de dimensão finita, temos que $\overline{F(K)}$ é compacto e, portanto, $F$ é um operador compacto.

O próximo resultado afirma que o espectro de $L_{0}^{\infty}$ e o espectro de $L^{\infty}$ gozam das mesmas propriedades.

Proposição 4.2.2 Sejam $L_{0}^{\infty}$ e $L^{\infty}$ definidos em (4.13) e (4.11), respectivamente, temos $\sigma\left(L_{0}^{\infty}\right)=$ $\sigma\left(L^{\infty}\right)$ e, além disso, 0 é um autovalor simples de $L^{\infty}$. Em particular, as propriedades do Lema 4.2.2 sẫo verdadeiras para $\sigma\left(L^{\infty}\right)$.

Demonstração. Considere $L^{\infty}$ na forma (4.12). Pelo item (ii) do Lema 4.2.2,

$$
L_{0}^{\infty}\left(\begin{array}{c}
\phi^{\prime} \\
\psi^{\prime}
\end{array}\right)=\left(\begin{array}{l}
0 \\
0
\end{array}\right)
$$

Além disso, usando integração por partes no termo $\left\langle\psi_{\infty}, \phi_{\infty}^{\prime \prime}\right\rangle$, podemos ver que

$$
\Pi^{\infty}\left(\phi_{\infty}^{\prime}, \psi_{\infty}^{\prime}\right)=\frac{2 \lambda\left(\phi_{\infty}, \psi_{\infty}\right)\left\langle\phi_{\infty}^{\prime}, \phi_{\infty}^{\prime \prime}\right\rangle+\left\langle\psi_{\infty}, \phi_{\infty}^{\prime \prime}\right\rangle+\left\langle\phi_{\infty}^{\prime}, \psi_{\infty}^{\prime}\right\rangle}{\left\|\phi_{\infty}^{\prime}\right\|^{2}}=0 .
$$

Portanto podemos concluir que 0 é autovalor de $L^{\infty}$ com autofunção associada $\left(\phi_{\infty}^{\prime}, \psi_{\infty}^{\prime}\right)$.

Para ver que 0 é autovalor simples, suponha que exista $(\bar{u}, \bar{w}) \in D\left(L^{\infty}\right)$ tal que

$$
L^{\infty}\left(\begin{array}{c}
\bar{u} \\
\bar{w}
\end{array}\right)=\left(\begin{array}{l}
0 \\
0
\end{array}\right)
$$

o que implica

$$
\left(\begin{array}{l}
0 \\
0
\end{array}\right)=L_{0}^{\infty}\left(\begin{array}{c}
\bar{u} \\
\bar{w}
\end{array}\right)+\Pi^{\infty}(\bar{u}, \bar{w})\left(\begin{array}{c}
\phi_{\infty}^{\prime} \\
\psi_{\infty}^{\prime}
\end{array}\right) .
$$

Se $\Pi^{\infty}(\bar{u}, \bar{w})=0$ então $(\bar{u}, \bar{w})$ é múltiplo de $\left(\phi_{\infty}^{\prime}, \psi_{\infty}^{\prime}\right)$, pois 0 é um autovalor simples de $L_{0}^{\infty}$.

Se $\Pi^{\infty}(\bar{u}, \bar{w}) \neq 0$ então

$$
L_{0}^{\infty}\left(\begin{array}{c}
\bar{u} \\
\bar{w}
\end{array}\right)=-\Pi^{\infty}(\bar{u}, \bar{w})\left(\begin{array}{c}
\phi_{\infty}^{\prime} \\
\psi_{\infty}^{\prime}
\end{array}\right) .
$$

Mas isto é impossível pelo item (ii) do Lema 4.2.2.

Para mostrar que $\sigma\left(L^{\infty}\right)=\sigma\left(L_{0}^{\infty}\right)$, vamos mostrar que os dois operadores possuem o mesmo resolvente, isto é, $\rho\left(L_{0}^{\infty}\right)=\rho\left(L^{\infty}\right)$.

$\left.1^{0}\right) \rho\left(L_{0}^{\infty}\right) \subset \rho\left(L^{\infty}\right):$ Se $\mu \in \rho\left(L_{0}^{\infty}\right)$ então $\left(L_{0}^{\infty}-\mu I\right)^{-1}$ existe, é limitada e definida em um conjunto denso $X \times Y$. 
Sejam $(U, W) \in X \times Y$ e $(\bar{m}, \bar{n})$ o único elemento de $D\left(L_{0}^{\infty}\right)$ tal que

$$
\left(L_{0}^{\infty}-\mu I\right)\left(\begin{array}{l}
\bar{m} \\
\bar{n}
\end{array}\right)=\left(\begin{array}{l}
U \\
W
\end{array}\right) .
$$

Se $\Pi^{\infty}(\bar{m}, \bar{n})=0$ então $\mu \in \rho\left(L^{\infty}\right)$, pois

$$
\left(L^{\infty}-\mu I\right)\left(\begin{array}{l}
\bar{m} \\
\bar{n}
\end{array}\right)=\left(L_{0}^{\infty}-\mu I\right)\left(\begin{array}{l}
\bar{m} \\
\bar{n}
\end{array}\right)=\left(\begin{array}{l}
U \\
W
\end{array}\right) .
$$

Suponha que $\Pi^{\infty}(\bar{m}, \bar{n}) \neq 0$ e considere

$$
\left(\begin{array}{c}
m^{*} \\
n^{*}
\end{array}\right)=\left(\begin{array}{c}
\bar{m} \\
\bar{n}
\end{array}\right)+\frac{1}{\mu} \Pi^{\infty}(\bar{u}, \bar{w})\left(\begin{array}{c}
\phi_{\infty}^{\prime} \\
\psi_{\infty}^{\prime}
\end{array}\right) .
$$

Como $L^{\infty}$ é linear,

$$
L^{\infty}\left(\begin{array}{c}
m^{*} \\
n^{*}
\end{array}\right)=L^{\infty}\left(\begin{array}{c}
\bar{m} \\
\bar{n}
\end{array}\right)+\frac{1}{\mu} \Pi^{\infty}(\bar{u}, \bar{w}) L^{\infty}\left(\begin{array}{c}
\phi_{\infty}^{\prime} \\
\psi_{\infty}^{\prime}
\end{array}\right) .
$$

Pela relação (4.12) e por $L^{\infty}\left(\begin{array}{c}\phi_{\infty}^{\prime} \\ \psi_{\infty}^{\prime}\end{array}\right)=\left(\begin{array}{l}0 \\ 0\end{array}\right)$ temos

$$
L^{\infty}\left(\begin{array}{c}
m^{*} \\
n^{*}
\end{array}\right)=L_{0}^{\infty}\left(\begin{array}{c}
\bar{m} \\
\bar{n}
\end{array}\right)+\Pi^{\infty}(\bar{u}, \bar{w})\left(\begin{array}{c}
\phi_{\infty}^{\prime} \\
\psi_{\infty}^{\prime}
\end{array}\right) .
$$

Por (4.14),

$$
\begin{aligned}
L^{\infty}\left(\begin{array}{c}
m^{*} \\
n^{*}
\end{array}\right) & =\left(\begin{array}{c}
U+\mu \bar{m}+\Pi^{\infty}(\bar{u}, \bar{w}) \phi_{\infty}^{\prime} \\
W+\mu \bar{n}+\Pi^{\infty}(\bar{u}, \bar{w}) \psi_{\infty}^{\prime}
\end{array}\right) \\
& =\left(\begin{array}{c}
U+\mu\left[\bar{m}+\frac{1}{\mu} \Pi^{\infty}(\bar{u}, \bar{w}) \phi_{\infty}^{\prime}\right] \\
W+\mu\left[\bar{n}+\frac{1}{\mu} \Pi^{\infty}(\bar{u}, \bar{w}) \psi_{\infty}^{\prime}\right]
\end{array}\right) \\
& =\left(\begin{array}{c}
U+\mu m^{*} \\
W+\mu n^{*}
\end{array}\right) .
\end{aligned}
$$

Logo, para qualquer $(U, W) \in X \times Y$, existe $\left(m^{*}, n^{*}\right) \in D\left(L^{\infty}\right)$ tal que

$$
\left(L^{\infty}-\mu I\right)\left(\begin{array}{l}
m^{*} \\
n^{*}
\end{array}\right)=\left(\begin{array}{l}
U \\
W
\end{array}\right)
$$

e, portanto, $\left(L^{\infty}-\mu I\right)$ é sobrejetora.

Suponha que existam dois elementos $\left(m_{1}^{*}, n_{1}^{*}\right),\left(m_{2}^{*}, n_{2}^{*}\right) \in D\left(L^{\infty}\right)$ tais que

$$
\left(L^{\infty}-\mu I\right)\left(\begin{array}{c}
m_{1}^{*} \\
n_{1}^{*}
\end{array}\right)=\left(\begin{array}{l}
U \\
W
\end{array}\right)
$$

e

$$
\left(L^{\infty}-\mu I\right)\left(\begin{array}{c}
m_{2}^{*} \\
n_{2}^{*}
\end{array}\right)=\left(\begin{array}{l}
U \\
W
\end{array}\right) .
$$

Usando a relação (4.12) podemos concluir que

$$
\left(L_{0}^{\infty}-\mu I\right)\left(\begin{array}{c}
m_{1}^{*} \\
n_{1}^{*}
\end{array}\right)=\left(\begin{array}{c}
U \\
W
\end{array}\right)-\Pi^{\infty}\left(m_{1}^{*}, n_{1}^{*}\right)\left(\begin{array}{c}
\phi_{\infty}^{\prime} \\
\psi_{\infty}^{\prime}
\end{array}\right)
$$


e, além disso, como

$$
\Pi^{\infty}\left(m_{2}^{*}-m_{1}^{*}, n_{2}^{*}-n_{1}^{*}\right)=\Pi^{\infty}\left(m_{2}^{*}, n_{2}^{*}\right)-\Pi^{\infty}\left(m_{1}^{*}, n_{1}^{*}\right),
$$

obtemos

$$
\left(L_{0}^{\infty}-\mu I\right)\left(\begin{array}{c}
m_{1}^{*}-m_{2}^{*} \\
n_{1}^{*}-n_{2}^{*}
\end{array}\right)=\Pi^{\infty}\left(m_{2}^{*}, n_{2}^{*}\right)-\Pi^{\infty}\left(m_{1}^{*}, n_{1}^{*}\right)\left(\begin{array}{c}
\phi_{\infty}^{\prime} \\
\psi_{\infty}^{\prime}
\end{array}\right) .
$$

$\mathrm{Se}$

$$
\Pi^{\infty}\left(m_{2}^{*}, n_{2}^{*}\right)=\Pi^{\infty}\left(m_{1}^{*}, n_{1}^{*}\right),
$$

então $\left(L^{\infty}-\mu I\right)$ é injetora, pois $\left(L_{0}^{\infty}-\mu I\right)$ é injetora.

Se

$$
\Pi^{\infty}\left(m_{2}^{*}, n_{2}^{*}\right) \neq \Pi^{\infty}\left(m_{1}^{*}, n_{1}^{*}\right),
$$

então

$$
\left(L_{0}^{\infty}-\mu I\right)\left(\begin{array}{c}
\bar{m} \\
\bar{n}
\end{array}\right)=-\Pi^{\infty}(\bar{m}, \bar{n})\left(\begin{array}{c}
\phi_{\infty}^{\prime} \\
\psi_{\infty}^{\prime}
\end{array}\right)
$$

onde

$$
\begin{gathered}
\bar{m}=m_{2}^{*}-m_{1}^{*}, \\
\bar{n}=n_{2}^{*}-n_{1}^{*} .
\end{gathered}
$$

Como $\left(L_{0}^{\infty}-\mu I\right)^{-1}$ existe, podemos escrever

$$
\left(\begin{array}{l}
\bar{m} \\
\bar{n}
\end{array}\right)=-\Pi^{\infty}(\bar{m}, \bar{n})\left(L_{0}^{\infty}-\mu I\right)^{-1}\left(\begin{array}{c}
\phi_{\infty}^{\prime} \\
\psi_{\infty}^{\prime}
\end{array}\right)
$$

Usando a igualdade

$$
\left(L_{0}^{\infty}-\mu I\right)^{-1}\left(\begin{array}{c}
\phi_{\infty}^{\prime} \\
\psi_{\infty}^{\prime}
\end{array}\right)=-\frac{1}{\mu}\left(\begin{array}{c}
\phi_{\infty}^{\prime} \\
\psi_{\infty}^{\prime}
\end{array}\right)
$$

vemos que $(\bar{m}, \bar{n})$ é uma combinação linear de $\left(\phi_{\infty}^{\prime}, \psi_{\infty}^{\prime}\right)$, logo

$$
\Pi^{\infty}(\bar{m}, \bar{n})=0
$$

o que é uma contradição. Logo $\left(L^{\infty}-\mu I\right)$ é injetora e concluímos que $\left(L^{\infty}-\mu I\right)^{-1}$ existe.

Agora, $\left(L^{\infty}-\mu I\right)^{-1}$ é limitada, pois as igualdades (4.14) e (4.15) nos permitem concluir que

$$
\begin{aligned}
\left(L^{\infty}-\mu I\right)^{-1}\left(\begin{array}{c}
U \\
W
\end{array}\right) & =\left(\begin{array}{l}
m^{*} \\
n^{*}
\end{array}\right) \\
& =\left(\begin{array}{l}
\bar{m} \\
\bar{n}
\end{array}\right)+\Pi^{\infty}(\bar{u}, \bar{w})\left(\begin{array}{c}
\phi_{\infty}^{\prime} \\
\psi_{\infty}^{\prime}
\end{array}\right) \\
& =\left(L_{0}^{\infty}-\mu I\right)^{-1}\left(\begin{array}{c}
U \\
W
\end{array}\right)+\frac{1}{\mu} \Pi^{\infty}(\bar{u}, \bar{w})\left(\begin{array}{c}
\phi_{\infty}^{\prime} \\
\psi_{\infty}^{\prime}
\end{array}\right)
\end{aligned}
$$

e concluímos que $\rho\left(L_{0}^{\infty}\right) \subset \rho\left(L^{\infty}\right)$. A outra inclusão segue o mesmo raciocínio usando a igualdade

$$
L_{0}^{\infty}\left(\begin{array}{c}
m \\
n
\end{array}\right)=L^{\infty}\left(\begin{array}{c}
m \\
n
\end{array}\right)-\Pi^{\infty}(m, n)\left(\begin{array}{c}
\phi_{\infty}^{\prime} \\
\psi_{\infty}^{\prime}
\end{array}\right)
$$

portanto 0 é um autovalor simples de $L^{\infty}$.

Além disso, como $\Pi^{\infty}(m, n)\left(\begin{array}{c}\phi_{\infty}^{\prime} \\ \psi_{\infty}^{\prime}\end{array}\right)$ é um operador compacto de posto 1, pelo Lema 1.2.1, $\sigma_{e}\left(L^{\infty}\right)=\sigma_{e}\left(L_{0}^{\infty}\right)$. 


\section{Capítulo 5}

\section{Convergência espectral}

\subsection{Definições e resultados básicos}

Sejam $E$ e $F$ espaços de Banach separáveis e sejam $\left\{E_{r}\right\}_{r>0}$ e $\left\{F_{r}\right\}_{r>0}$ famílias de espaços de Banach separáveis. Sejam $\left\{m_{r}\right\}_{r>0}, m_{r} \in \mathcal{L}\left(E, E_{r}\right)$ e $\left\{n_{r}\right\}_{r>0}, n_{r} \in \mathcal{L}\left(F, F_{r}\right)$ operadores lineares limitados tais que:

$$
\begin{aligned}
& \lim _{r \rightarrow \infty}\left\|m_{r} e\right\|_{E_{r}} \rightarrow\|e\|_{E}, \quad \text { para cada } e \in E \\
& \lim _{r \rightarrow \infty}\left\|n_{r} f\right\|_{F_{r}} \rightarrow\|f\|_{F}, \quad \text { para cada } f \in F .
\end{aligned}
$$

Definição 5.1.1 Uma família $\left\{e_{r}\right\}_{r>0}, e_{r} \in E_{r}$, é $\mathcal{M}$-convergente para $e \in E$, se

$$
\lim _{r \rightarrow \infty}\left\|e_{r}-m_{r} e\right\|_{E_{r}}=0
$$

Neste caso, denotamos por $e_{r} \stackrel{\mathcal{M}}{\rightarrow}$ e. A definição de $\mathcal{N}$-convergência é análoga.

Definição 5.1.2 Uma família $\left\{e_{r}\right\}_{r>0}, e_{r} \in E_{r}$, é $\mathcal{M}$-compacta se cada sequência infinita contém uma subsequência $\mathcal{M}$-convergente. A definição de $\mathcal{N}$-compacidade é análoga.

Definição 5.1.3 Uma família de operadores lineares limitados $\left\{T_{r}\right\}_{r>0}, T_{r} \in \mathcal{L}\left(E_{r}, F_{r}\right)$ é $\mathcal{M N}$-convergente $a T \in \mathcal{L}(E, F)$ se

$$
e_{r} \stackrel{\mathcal{M}}{\rightarrow} e \quad \Longrightarrow \quad T_{r} e_{r}, \stackrel{\mathcal{N}}{\rightarrow} T e \quad \text { quando } r \rightarrow \infty .
$$

Neste caso, denotamos $T_{r} \stackrel{\mathcal{M N}}{\rightarrow} T$.

Definição 5.1.4 Dizemos que $T_{r} \mathcal{M N}$ - converge regularmente para $T$ se, e somente se, $T_{r} \stackrel{\mathcal{M N}}{\rightarrow} T$ e se para cada sequência limitada $\left\{e_{r}\right\}_{r>0}, e_{r} \in E_{r}$, tal que a sequência $\left\{T_{r} e_{r}\right\}_{r>0}$ é $\mathcal{N}$-compacta implica que a sequência $\left\{e_{r}\right\}_{r>0}$ é $\mathcal{M}$-compacta.

Definição 5.1.5 Sejam $X$ e $Y$ espaços de Banach e $T: X \rightarrow Y$ um operador linear. $T$ é um operador de Fredholm se $\operatorname{Ker}(T)$ e coKer $(T):=Y / \operatorname{Im}(T)$ têm dimensão finita.

$O$ indice de um operador de Fredholm é definido por

$$
\operatorname{ind}(T)=\operatorname{dimKer}(T)-\operatorname{dim}(\operatorname{coKer}(T)) .
$$

Teorema 5.1.1 Seja a família de operadores $T(s)=T-s R \in \mathcal{L}(E, F)$ e $T_{r}(s)=T_{r}-s R_{r} \in$ $\mathcal{L}\left(E_{r}, F_{r}\right)$, onde o parâmetro $s \in S$, com $S$ um subconjunto limitado do plano complexo $\mathbb{C}$, que satisfaz as seguintes hipóteses:

(i) $T_{r}(s) \mathcal{M N}$-converge regularmente para $T(s)$, para todo $s \in S$,

(ii) Para cada $s \in S$, o operador $T_{r}(s)$ e $T(s)$ são Fredholm com indice 0,

(iii) Existe $s^{\prime} \in S$ tal que $\operatorname{Ker}\left(T\left(s^{\prime}\right)\right)=\{0\}$, 
(iv) Existe uma constante $C=C(S)>0$ tal que $\left\|T_{r}(s)\right\|_{\mathcal{L}\left(E_{r}, F_{r}\right)} \leq C$, para todo $r \geq 0$.

Então, se denotamos por $W\left(s_{0}\right)$ o subespaço associado a $T\left(s_{0}\right)$, isto é, o subespaço gerado pela cadeia de vetores $\left\{e_{0}, e_{1}, \ldots, e_{k}, \ldots\right\}$ definido como

$$
\left(T-s_{0} R\right) e_{0}=0, \quad\left(T-s_{0} R\right) e_{1}=R e_{0}, \quad \ldots \quad\left(T-s_{0} R\right) e_{k}=R e_{k-1}, \quad \ldots
$$

e se denotamos por $W_{r}\left(s_{0}, \delta\right)$ todos os subespaços associado a $T_{r}(s)$ para todo $\left|s-s_{0}\right| \leq \delta, s \in S$, então temos que, para $\delta>0$ pequeno o bastante,

$$
\operatorname{dist}_{H}\left(W_{r}\left(s_{0}, \delta\right), W\left(s_{0}\right)\right) \rightarrow 0, \quad \text { quando } \quad r \rightarrow+\infty
$$

$e$, portanto, existe um $\delta>0$ pequeno, tal que

$$
\operatorname{dim}\left(W_{r}\left(s_{0}, \delta\right)\right)=\operatorname{dim} W\left(s_{0}\right), \quad \text { quando } \quad r \rightarrow+\infty .
$$

Demonstração. Veja Vainikko (1979)

Teorema 5.1.2 Se $T: X \rightarrow Y$ é de Fredholm e $S: X \rightarrow Y$ é um operador linear compacto, então $T+S$ é um operador de Fredholm com indice

$$
\operatorname{ind}(T+S)=\operatorname{ind}(T)
$$

Demonstração. Veja Gohberg et al. (2012)

\subsection{Convergência espectral}

Vamos considerar $E=H^{1}\left(\mathbb{R}, \mathbb{C}^{3}\right)$ e $F=L^{2}\left(\mathbb{R}, \mathbb{C}^{3}\right)$ e, para um intervalo finito $J_{r}$, considere os espaços $E_{r}=H^{1}\left(J_{r}, \mathbb{C}^{3}\right)$ e $F_{r}=L^{2}\left(J_{r}, \mathbb{C}^{3}\right) \times \mathbb{C}^{3}$. Para $E, F$ e $E_{r}$ consideramos as normas usuais e para $F_{r}$ consideramos a norma

$$
\|(f, \eta)\|_{F_{r}}=\|f\|_{L^{2}\left(J_{r}\right)}+|\eta| .
$$

Definimos a família de operadores lineares $m_{r}: E \rightarrow E_{r}$ e $n_{r}: F \rightarrow F_{r}$ tais que

$$
\begin{aligned}
m_{r}\left(\begin{array}{c}
u \\
v \\
w
\end{array}\right) & =\left(\begin{array}{c}
u_{\left.\right|_{J_{r}}} \\
v_{\left.\right|_{J_{r}}} \\
w_{\left.\right|_{J_{r}}}
\end{array}\right), \\
n_{r}\left(\begin{array}{c}
u \\
v \\
w
\end{array}\right) & =\left(\begin{array}{c}
u_{\left.\right|_{J_{r}}} \\
v_{J_{r}} \\
\left.w\right|_{J_{r}} \\
0 \\
0 \\
0
\end{array}\right) .
\end{aligned}
$$

Considere a família de operadores $T_{\infty}^{s}, T_{0, \infty}^{s}, \Pi_{\infty}: E \rightarrow F$ definidos como

$$
\begin{aligned}
T_{\infty}^{s}\left(\begin{array}{c}
u \\
v \\
w
\end{array}\right)= & \left(\begin{array}{c}
u_{x} \\
v_{x} \\
w_{x}
\end{array}\right)+\left(\begin{array}{ccc}
0 & -1 & 0 \\
f^{\prime}\left(\phi_{\infty}\right)-s & -\lambda^{\infty} & -1 \\
-\frac{\varepsilon}{\lambda^{\infty}} & 0 & \frac{\varepsilon \gamma+s}{\lambda^{\infty}}
\end{array}\right)\left(\begin{array}{c}
u \\
v \\
w
\end{array}\right)+\left(\begin{array}{c}
0 \\
\phi_{\infty}^{\prime} \Pi^{\infty}(u, w) \\
-\frac{1}{\lambda^{\infty}} \psi_{\infty}^{\prime} \Pi^{\infty}(u, w)
\end{array}\right), \\
& T_{0, \infty}^{s}\left(\begin{array}{c}
u \\
v \\
w
\end{array}\right)=\left(\begin{array}{c}
u_{x} \\
v_{x} \\
w_{x}
\end{array}\right)+\left(\begin{array}{ccc}
0 & -1 & 0 \\
f^{\prime}\left(\phi_{\infty}\right)-s & -\lambda^{\infty} & -1 \\
-\frac{\varepsilon}{\lambda^{\infty}} & 0 & \frac{\varepsilon \gamma+s}{\lambda^{\infty}}
\end{array}\right)\left(\begin{array}{c}
u \\
v \\
w
\end{array}\right)
\end{aligned}
$$


e

$$
\Pi_{\infty}\left(\begin{array}{c}
u \\
v \\
w
\end{array}\right)=\left(\begin{array}{c}
0 \\
\phi_{\infty}^{\prime} \Pi^{\infty}(u, w) \\
-\frac{1}{\lambda^{\infty}} \psi_{\infty}^{\prime} \Pi^{\infty}(u, w)
\end{array}\right) .
$$

Observe que $T_{\infty}^{s}=T_{0, \infty}^{s}+\Pi_{\infty}$. Além disso, o operador $T_{0, \infty}^{s}$ é um operador local. O operador $T_{\infty}^{s}$ pode ser decomposto como

$$
T_{\infty}^{s}=T_{\infty}^{0}-s R_{\infty}
$$

onde

$$
R_{\infty}\left(\begin{array}{c}
u \\
v \\
w
\end{array}\right)=\left(\begin{array}{c}
0 \\
u \\
-\frac{w}{\lambda^{\infty}}
\end{array}\right) .
$$

Definimos os operadores em um intervalo limitado, $T_{r}^{s}, T_{0, r}^{s}$, por $\Pi_{r}: E_{r} \rightarrow F_{r}$

$$
\begin{aligned}
T_{r}^{s}\left(\begin{array}{c}
u \\
v \\
w
\end{array}\right)=\left(\begin{array}{c}
u_{x} \\
v_{x} \\
w_{x} \\
0 \\
0 \\
0
\end{array}\right)+\left(\begin{array}{ccc}
0 & -1 & 0 \\
f^{\prime}\left(\phi_{r}\right)-s & -\lambda^{r} & -1 \\
-\frac{\varepsilon}{\lambda^{r}} & 0 & \frac{\varepsilon \gamma+s}{\lambda^{r}} \\
0 & 0 & 0 \\
0 & 0 & 0 \\
0 & 0 & 0
\end{array}\right)\left(\begin{array}{c}
u \\
v \\
w
\end{array}\right)+\left(\begin{array}{c}
0 \\
\phi_{r}^{\prime} \Pi^{r}(u, w) \\
1 \\
-\frac{1}{\lambda^{r}} \psi_{r}^{\prime} \Pi^{r}(u, w) \\
0 \\
0 \\
0
\end{array}\right)+\left(\begin{array}{c}
0 \\
0 \\
0 \\
u\left(x_{-}\right) \\
u\left(x_{+}\right) \\
w\left(x_{-}\right)
\end{array}\right), \\
T_{0, r}^{s}\left(\begin{array}{c}
u \\
v \\
w
\end{array}\right)=\left(\begin{array}{c}
u_{x} \\
v_{x} \\
w_{x} \\
0 \\
0 \\
0
\end{array}\right)+\left(\begin{array}{ccc}
0 & -1 & 0 \\
f^{\prime}\left(\phi_{r}\right)-s & -\lambda^{r} & -1 \\
-\frac{\varepsilon}{\lambda^{r}} & 0 & \frac{\varepsilon \gamma+s}{\lambda^{r}} \\
0 & 0 & 0 \\
0 & 0 & 0 \\
0 & 0 & 0
\end{array}\right)\left(\begin{array}{c}
u \\
v \\
w
\end{array}\right)+\left(\begin{array}{c}
0 \\
0 \\
0 \\
u\left(x_{-}\right) \\
u\left(x_{+}\right) \\
w\left(x_{-}\right)
\end{array}\right)
\end{aligned}
$$

e

$$
\Pi_{r}\left(\begin{array}{c}
u \\
v \\
w
\end{array}\right)=\left(\begin{array}{c}
0 \\
\phi_{r}^{\prime} \Pi^{r}(u, w) \\
-\frac{1}{\lambda^{r}} \psi_{r}^{\prime} \Pi^{r}(u, w) \\
0 \\
0 \\
0
\end{array}\right)
$$

de forma similar temos a igualdade $T_{r}^{s}=T_{r}^{0}-s R_{r}$ com

$$
R_{r}\left(\begin{array}{c}
u \\
v \\
w
\end{array}\right)=\left(\begin{array}{c}
0 \\
u \\
-\frac{w}{\lambda^{r}} \\
0 \\
0 \\
0
\end{array}\right)
$$

Teorema 5.2.1 Considere o operador diferencial linear $T: E \rightarrow F$ definido por $T z:=z_{x}-M(\cdot) z$ 
e a sequência de operadores lineares limitados em um intervalo finito $T_{r}: E_{r} \rightarrow F_{r}$ definido por

$$
T_{r} z:=\left(\begin{array}{c}
z_{x}-M(\cdot) z \\
R z
\end{array}\right)
$$

Se $D \neq 0^{1}$ então $T_{r} \stackrel{\mathcal{M N}}{\rightarrow} T$ regularmente, quando $r \rightarrow \infty$.

Demonstração. Veja Beyn e Rottmann-Matthes (2007), pág 6.

Proposição 5.2.1 Com a notação acima, para qualquer $s \in\{s \in \mathbb{C} ; \operatorname{Re} s>-\delta\}$ temos:

(i) A sequência de operadores $T_{0, r}^{s} \mathcal{M N}$-converge regularmente para $T_{0, \infty}^{s}$, quando $r \rightarrow \infty$.

(ii)A sequência de operadores $T_{r}^{s} \mathcal{M N}$-converge regularmente para $T_{\infty}^{s}$, quando $r \rightarrow \infty$.

(iii) Os operadores $T_{\infty}^{s}$ e $T_{r}^{s}$ são operadores Fredholm de indice 0.

Demonstração. (i) Considere o operador $\tilde{T}_{0, r}^{s}: E_{r} \rightarrow F_{r}$ definido por

$$
\tilde{T}_{0, r}^{s}\left(\begin{array}{c}
u \\
v \\
w
\end{array}\right)=\left(\begin{array}{c}
u_{x} \\
v_{x} \\
w_{x} \\
0 \\
0 \\
0
\end{array}\right)+\left(\begin{array}{ccc}
0 & -1 & 0 \\
f^{\prime}\left(\phi_{\infty}\right)-s & -\lambda^{\infty} & -1 \\
-\frac{\varepsilon}{\lambda^{\infty}} & 0 & \frac{\varepsilon \gamma+s}{\lambda^{\infty}} \\
0 & 0 & 0 \\
0 & 0 & 0 \\
0 & 0 & 0
\end{array}\right)\left(\begin{array}{c}
u \\
v \\
w
\end{array}\right)+\left(\begin{array}{c}
0 \\
0 \\
0 \\
u\left(x_{-}\right) \\
u\left(x_{+}\right) \\
w\left(x_{-}\right)
\end{array}\right),
$$

onde $f^{\prime}\left(\phi_{\infty}\right)$ está restrita ao intervalo $J_{r}$. Pelo Teorema 5.2.1, sabemos que $\tilde{T}_{0, r}^{s} \mathcal{M N}$-converge regularmente para $T_{0, \infty}^{s}$

Seja

$$
\tilde{R}_{r}=\left(\begin{array}{ccc}
0 & 0 & 0 \\
-f^{\prime}\left(\phi_{\infty}\right)+f^{\prime}\left(\phi_{r}^{\prime}\right) & \lambda^{\infty}-\lambda^{r} & 0 \\
\frac{\varepsilon}{\lambda^{\infty}}-\frac{\varepsilon}{\lambda^{r}} & 0 & \frac{\varepsilon \gamma}{\lambda^{r}}-\frac{\varepsilon \gamma}{\lambda^{\infty}} \\
0 & 0 & 0 \\
0 & 0 & 0 \\
0 & 0 & 0
\end{array}\right)\left(\begin{array}{c}
u \\
v \\
w
\end{array}\right) .
$$

Portanto $T_{0, r}^{s}=\tilde{T}_{0, r}^{s}+\tilde{R}_{r}$.

Pelo Lema 4.1.2, quando $r \rightarrow \infty, \lambda^{r} \rightarrow \lambda^{\infty}$. Pelo Lema 4.1.3,

$$
\left|f^{\prime}\left(\phi_{\infty}\right)-f^{\prime}\left(\phi_{r}\right)\right| \rightarrow 0 \text { quando } r \rightarrow \infty,
$$

e, portanto, $\left\|\tilde{R}_{r}\right\|_{\mathcal{L}\left(E_{r}, F_{r}\right)} \rightarrow 0$, quando $r \rightarrow \infty$.

Logo $T_{0, r}^{s} \mathcal{M N}$-converge regularmente para $T_{0, \infty}^{s}$, quando $r \rightarrow \infty$.

(ii) Segue das igualdades

$$
T_{r}^{s}=T_{0, r}^{s}+\Pi_{r} \quad \text { e } \quad T_{\infty}^{s}=T_{0, \infty}^{s}+\Pi_{\infty},
$$

do item (i) deste lema e do fato que $\Pi_{r} \mathcal{M N}$-converge para $\Pi_{\infty}$.

(iii) $\left.1^{\circ}\right) T_{r}^{s}$ é Fredholm com índice 0. De fato,

\footnotetext{
${ }^{1}$ Relembre as definições de R e D em (3.7) e (3.8)
} 
Considere o operador $D_{r}: E_{r} \rightarrow F_{r}$ dado por

$$
D_{r}\left(\begin{array}{c}
u \\
v \\
w
\end{array}\right)=\left(\begin{array}{c}
u_{x} \\
v_{x} \\
w_{x} \\
0 \\
0 \\
0
\end{array}\right)
$$

A dimensão do $\operatorname{Ker}\left(D_{r}\right)=3$, pois $\operatorname{Ker}\left(D_{r}\right)=\left\{(u, v, w) \in E_{r}: u, v\right.$ e $w$ são constantes $\} \equiv \mathbb{C} \times \mathbb{C} \times \mathbb{C}$ e como a imagem de $D_{r}$ é $L^{2}\left(J_{r}\right) \times L^{2}\left(J_{r}\right) \times L^{2}\left(J_{r}\right) \times\{0\} \times\{0\} \times\{0\} \subset F_{r}$ e tem codimensão 3 , segue que $D_{r}$ é operador de Fredholm com índice 0.

Agora, considere o operador $T_{0, r}^{s}-D_{r}: E_{r} \rightarrow F_{r}$, isto é,

$$
\left(T_{0, r}^{s}-D_{r}\right)\left(\begin{array}{c}
u \\
v \\
w
\end{array}\right)=\left(\begin{array}{ccc}
0 & -1 & 0 \\
f^{\prime}\left(\phi_{r}\right)-s & -\lambda^{r} & -1 \\
-\frac{\varepsilon}{\lambda^{r}} & 0 & \frac{\varepsilon \gamma+s}{\lambda^{r}} \\
0 & 0 & 0 \\
0 & 0 & 0 \\
0 & 0 & 0
\end{array}\right)\left(\begin{array}{c}
u \\
v \\
w
\end{array}\right)+\left(\begin{array}{c}
0 \\
0 \\
0 \\
u\left(x_{-}\right) \\
u\left(x_{+}\right) \\
w\left(x_{-}\right)
\end{array}\right) .
$$

Como $T_{0, r}^{s}-D_{r}$ é um operador limitado de $L^{2}\left(J_{r}, \mathbb{C}\right) \times L^{2}\left(J_{r}, \mathbb{C}\right) \times L^{2}\left(J_{r}, \mathbb{C}\right)$ em $F_{r}, \operatorname{logo} T_{0, r}^{s}-D_{r}$ é um operador compacto de $E_{r}$ em $F_{r}$. Pelo Teorema 5.1.2, $T_{0, r}^{s}$ é um operador de Fredholm de índice 0 .

Como $T_{r}^{s}=T_{0, r}^{s}+\Pi_{r}$ e $\Pi_{r}$ é um operador compacto então $T_{r}^{s}$ é um operador de Fredholm de índice 0 .

$\left.2^{\circ}\right) T_{\infty}^{s}$ é Fredholm de índice 0 . De fato, seja $T_{\infty}^{s}=P_{\infty}^{s}+K_{\infty}+\Pi_{\infty}$, onde $\Pi_{\infty}$ foi definido em $(5.3)$,

$$
K_{\infty}\left(\begin{array}{c}
u \\
v \\
w
\end{array}\right)=\left(\begin{array}{c}
0 \\
{\left[f^{\prime}\left(\phi_{\infty}\right)-V(\cdot)\right] u} \\
0
\end{array}\right)
$$

$\mathrm{e}$

$$
P_{\infty}^{s}\left(\begin{array}{c}
u \\
v \\
w
\end{array}\right)=\left(\begin{array}{c}
u_{x} \\
v_{x} \\
w_{x}
\end{array}\right)+\left(\begin{array}{ccc}
0 & -1 & 0 \\
V(\cdot)-s & -\lambda^{\infty} & -1 \\
-\frac{\varepsilon}{\lambda^{\infty}} & 0 & \frac{\varepsilon \gamma+s}{\lambda^{\infty}}
\end{array}\right)\left(\begin{array}{c}
u \\
v \\
w
\end{array}\right) .
$$

O termo $V$ que aparece em $P_{\infty}^{s}$ é definido por

$$
V(x)=\left\{\begin{array}{ll}
f^{\prime}(0), & \text { se } x \in(-\infty, 0] \\
f^{\prime}\left(u_{2}\right), & \text { se } x \in(0,+\infty)
\end{array} .\right.
$$

Como $f^{\prime}\left(\phi_{\infty}(x)\right)-V(x) \rightarrow 0$ quando $x \rightarrow \pm \infty$, concluímos que $K_{\infty}: E \rightarrow F$ é um operador compacto. Como $\Pi_{\infty}$ também é um operador compacto, a ideia é mostrar que $P_{\infty}^{s}$ é um operador de Fredholm de índice 0, e usar o Teorema 5.1.2 para concluir a demonstração.

Para mostrar que o operador $P_{\infty}^{s}$ é Fredholm de índice 0 precisamos que $\operatorname{Ker}\left(P_{\infty}^{s}\right)=\{0\}$ e $\operatorname{Im}\left(P_{\infty}^{s}\right)=L^{2}\left(\mathbb{R}, \mathbb{C}^{3}\right)$.

Podemos escrever $P_{\infty}^{s}$ na forma

$$
P_{\infty}^{s}\left(\begin{array}{c}
u \\
v \\
w
\end{array}\right)=\left(\begin{array}{c}
u_{x} \\
v_{x} \\
w_{x}
\end{array}\right)+M(x, s)\left(\begin{array}{c}
u \\
v \\
w
\end{array}\right)
$$


onde

$$
M(x, s)=M_{-}(s)=\left(\begin{array}{ccc}
0 & -1 & 0 \\
f^{\prime}(0)-s & -\lambda^{\infty} & -1 \\
-\frac{\varepsilon}{\lambda^{\infty}} & 0 & \frac{\varepsilon \gamma+s}{\lambda^{\infty}}
\end{array}\right), \quad x<0
$$

e

$$
M(x, s)=M_{+}(s)=\left(\begin{array}{ccc}
0 & -1 & 0 \\
f^{\prime}\left(u_{2}\right)-s & -\lambda^{\infty} & -1 \\
-\frac{\varepsilon}{\lambda^{\infty}} & 0 & \frac{\varepsilon \gamma+s}{\lambda^{\infty}}
\end{array}\right), \quad x>0 .
$$

Seja $(u, v, w) \in H^{1}\left(\mathbb{R}, \mathbb{C}^{3}\right)$ tal que

$$
P_{\infty}^{s}\left(\begin{array}{c}
u \\
v \\
w
\end{array}\right)=\left(\begin{array}{l}
0 \\
0 \\
0
\end{array}\right)
$$

Se considerarmos $x>0,(5.7)$ é uma EDO linear com coeficientes constantes e como $(u, v, w)$ são funções limitadas, o comportamento de sua solução quando $x \rightarrow \infty$, é determinado pelos autovalores e os correspondentes autovetores da matriz $M_{+}(s)$. Analogamente, se considerarmos $x<0$.

Mostramos no Lema 1.2.3 que, para $\varepsilon>0$ suficientemente pequeno, existe $\delta>0$ tal que, se Re $s>-\delta$, as matrizes $M_{+}(s)$ e $M_{-}(s)$ não tem autovalores no eixo imaginário e seus autovalores satisfazem:

$\operatorname{Re} \rho_{3}^{ \pm}<\operatorname{Re} \rho_{2}^{ \pm}<0<\operatorname{Re} \rho_{1}^{ \pm}$e seus respectivos autovetores são dados por

$$
\left(1,-\rho_{i}^{ \pm}, \frac{\varepsilon}{\rho_{i}^{ \pm} \lambda^{\infty}-s-\varepsilon \gamma}\right)
$$

Logo a solução $(u(x), v(x), w(x))$ de (5.7) satisfaz

$$
\left(\begin{array}{c}
u(x) \\
v(x) \\
w(x)
\end{array}\right)=C_{2} e^{\rho_{2}^{-} x}\left(\begin{array}{c}
1 \\
-\rho_{2}^{-} \\
\frac{\varepsilon}{\rho_{2}^{-} \lambda^{\infty}-s-\varepsilon \gamma}
\end{array}\right)+C_{3} e^{\rho_{3}^{-} x}\left(\begin{array}{c}
1 \\
-\rho_{3}^{-} \\
\frac{\varepsilon}{\rho_{3}^{-} \lambda^{\infty}-s-\varepsilon \gamma}
\end{array}\right), \quad x>0
$$

e

$$
\left(\begin{array}{c}
u(x) \\
v(x) \\
w(x)
\end{array}\right)=C_{1} e^{\rho_{1}^{+} x}\left(\begin{array}{c}
1 \\
-\rho_{1}^{+} \\
\frac{\varepsilon}{\rho_{1}^{+} \lambda^{\infty}-s-\varepsilon \gamma}
\end{array}\right), \quad x<0
$$

onde $C_{i} \in \mathbb{C}, i=1,2,3$, são constantes. Como $(u, v, w) \in H^{1}\left(\mathbb{R}, \mathbb{C}^{3}\right)$, devemos ter

$$
C_{2}\left(\begin{array}{c}
1 \\
-\rho_{2}^{-} \\
\frac{\varepsilon}{\rho_{2}^{-} \lambda^{\infty}-s-\varepsilon \gamma}
\end{array}\right)+C_{3}\left(\begin{array}{c}
1 \\
-\rho_{3}^{-} \\
\frac{\varepsilon}{\rho_{3}^{-} \lambda^{\infty}-s-\varepsilon \gamma}
\end{array}\right)=C_{1}\left(\begin{array}{c}
1 \\
-\rho_{1}^{+} \\
\frac{\varepsilon}{\rho_{1}^{+} \lambda^{\infty}-s-\varepsilon \gamma}
\end{array}\right) .
$$


Das igualdades

$$
\left\{\begin{array}{l}
C_{2}+C_{3}=C_{1} \\
-C_{2} \rho_{2}^{-}-C_{3} \rho_{3}^{-}=-C_{1} \rho_{1}^{+}
\end{array}\right.
$$

temos

$$
C_{2}\left(-\rho_{2}^{-}+\rho_{1}^{+}\right)=C_{3}\left(\rho_{3}^{-}-\rho_{1}^{+}\right) \text {. }
$$

Simplificando a expressão

$$
\frac{C_{2} \varepsilon}{\rho_{2}^{-} \lambda^{\infty}-s-\varepsilon \gamma}+\frac{C_{3} \varepsilon}{\rho_{3}^{-} \lambda^{\infty}-s-\varepsilon \gamma}=\frac{C_{1} \varepsilon}{\rho_{1}^{+} \lambda^{\infty}-s-\varepsilon \gamma}
$$

e usando o fato de $C_{2}+C_{3}=C_{1}$, obtemos

$$
C_{2}\left(\rho_{3}^{-} \lambda^{\infty}-s-\varepsilon \gamma\right)\left(\lambda^{\infty}\left(-\rho_{2}^{-}+\rho_{1}^{+}\right)\right)+C_{3}\left(\rho_{2}^{-} \lambda^{\infty}-s-\varepsilon \gamma\right)\left(\lambda^{\infty}\left(\rho_{1}^{+}-\rho_{3}^{-}\right)\right)=0 .
$$

Pela igualdade (5.9) concluímos

$$
\left[C_{3} \lambda^{\infty}\left(\rho_{1}^{+}-\rho_{2}^{-}\right)\right]\left[\lambda^{\infty}\left(\rho_{3}^{-}-\rho_{2}^{-}\right)\right]=0 .
$$

Como $\lambda^{\infty} \neq 0, \rho_{1}^{+} \neq \rho_{2}^{-}$e $\rho_{3}^{-} \neq \rho_{2}^{-}$, temos que $C_{3}=0$.

Das igualdades em (5.8) concluímos que $C_{1}=C_{2}=0$ e, portanto, $\operatorname{Ker}\left(P_{\infty}^{s}\right)=\{0\}$.

Pelo Lema 1 pag 137 de Henry (1981) podemos concluir que $\operatorname{Im}\left(P_{\infty}^{s}\right)=L^{2}\left(\mathbb{R}, \mathbb{C}^{3}\right)$.

Teorema 5.2.2 Existe $R_{0}>0$ tal que, para todo $r>r_{0}$, temos

$$
\sigma\left(L^{r}\right) \cap\left\{s \in \mathbb{C} ;|s| \leq r_{0}, \text { Re } s>-\delta\right\}=\{s(r)\} .
$$

Além disso, $s(r)<0$ é um autovalor simples de $L^{r}$ e $s(r) \rightarrow 0$, quando $r \rightarrow+\infty$.

Demonstração. Seja $s$ tal que $\operatorname{Re} s>-\delta . \operatorname{Ker}\left(T_{\infty}^{s}\right) \neq\{0\}$ se, e somente se, $s$ é um autovalor de $L^{\infty}$. Pela Proposição 4.2.2, $\sigma\left(L^{\infty}\right)=\sigma\left(L_{0}^{\infty}\right)$.

Podemos dividir a região $\{z \in \mathbb{C} ;$ Re $s>-\delta\}$ em dois subconjuntos

$$
C_{1} \equiv\left\{s \in \mathbb{C} ;|s| \leq R_{0}, \text { Re } s>-\delta\right\}
$$

e

$$
C_{2} \equiv\left\{s \in \mathbb{C} ;|s|>R_{0}, \text { Re } s>-\delta\right\} .
$$

Na demonstração do Teorema 1.3.3, vimos que, se $R_{0}>0$ é suficientemente grande, não existem autovalores de $L_{0}^{\infty}$ em $C_{2}$. No conjunto $C_{1}$, o único autovalor de $L_{0}^{\infty}$ é $s=0$. Para aplicar o Teorema 5.1.1 vamos considerar $s$ no subconjunto limitado $C_{1}$.

Pela Proposição 5.2.1 e os comentários acima, as hipóteses $(i)-(i v)$ são satisfeitas. Vamos então, calcular o subespaço $W\left(s_{0}\right)$, onde $s_{0}=0$ :

$e_{0}$ deve ser um vetor tal que $T_{\infty}^{0} e_{0}=0$. Como $\left(\phi_{\infty}^{\prime}, \psi_{\infty}^{\prime}\right)$ é autofunção de $L^{\infty}$ associada ao autovetor $s=0$, vamos tomar $e_{0}=\left(\phi_{\infty}^{\prime}, \phi_{\infty}^{\prime \prime}, \psi_{\infty}^{\prime}\right)$;

Para calcular $e_{1}$, precisamos resolver $T_{\infty}^{0} e_{1}=R_{\infty} e_{0}$, isto é,

$$
\left(\begin{array}{c}
u_{x} \\
v_{x} \\
w_{x}
\end{array}\right)+\left(\begin{array}{ccc}
0 & -1 & 0 \\
f^{\prime}\left(\phi_{\infty}\right) & -\lambda^{\infty} & -1 \\
-\frac{\varepsilon}{\lambda^{\infty}} & 0 & \frac{\varepsilon \gamma}{\lambda^{\infty}}
\end{array}\right)\left(\begin{array}{c}
u \\
v \\
w
\end{array}\right)+\left(\begin{array}{c}
0 \\
\phi_{\infty}^{\prime} \Pi^{\infty}(u, w) \\
\frac{-\psi_{\infty}^{\prime}}{\lambda^{\infty}} \Pi^{\infty}(u, w)
\end{array}\right)=\left(\begin{array}{c}
0 \\
\phi_{\infty}^{\prime} \\
\frac{-\psi_{\infty}^{\prime}}{\lambda^{\infty}}
\end{array}\right),
$$


ou seja,

$$
\left\{\begin{array}{l}
u_{x}-v=0 \\
v_{x}+f^{\prime}\left(\phi_{\infty}\right) u-\lambda^{\infty} v-w+\phi_{\infty}^{\prime} \Pi^{\infty}(u, w)=\phi_{\infty}^{\prime} . \\
w_{x}-\frac{\varepsilon}{\lambda^{\infty}}(u-\gamma w)-\frac{\psi_{\infty}^{\prime}}{\lambda^{\infty}} \Pi^{\infty}(u, w)=\frac{-\psi_{\infty}^{\prime}}{\lambda^{\infty}}
\end{array}\right.
$$

O problema acima é equivalente a

$$
\left(\begin{array}{c}
u_{x x}-\lambda^{\infty} u_{x}+f^{\prime}\left(\phi_{\infty}\right) u-w \\
-\lambda^{\infty} w_{x}-\varepsilon(u-\gamma w)
\end{array}\right)=\left(1-\Pi^{\infty}(u, w)\right)\left(\begin{array}{c}
\phi_{\infty}^{\prime} \\
\psi_{\infty}^{\prime}
\end{array}\right),
$$

ou seja, $L_{0}^{\infty}\left(\begin{array}{c}u \\ w\end{array}\right) \in\left[\left(\phi_{\infty}^{\prime}, \psi_{\infty}^{\prime}\right)\right]$. Como $\left(\phi_{\infty}^{\prime}, \psi_{\infty}^{\prime}\right)$ é uma autofunção de $L_{0}^{\infty}$ associada ao autovalor simples $0,(5.10)$ não tem solução, e portanto

$$
W(0)=\operatorname{span}\left\{\left(\begin{array}{c}
\phi_{\infty}^{\prime} \\
\psi_{\infty}^{\prime}
\end{array}\right)\right\} \quad \text { e } \quad \operatorname{dim}(W(0))=1 .
$$

Aplicando o Teorema 5.1.1 obtemos:

(i) Todos os valores $s(r) \in C_{1}$ tais que $\operatorname{Ker}\left(T_{r}^{s(r)}\right) \neq\{0\}$ satisfazem: $s(r) \rightarrow 0$, quando $r \rightarrow \infty$.

(ii) Existe $\delta>0$ pequeno tal que, para $r$ suficientemente grande, tem-se $\operatorname{dim}\left(W_{r}(0, \delta)\right)=1$.

Afirmação: $s(r)$ é um número real:

Se $s(r)$ for um número complexo, então seu complexo conjugado $\overline{s(r)}$ deve satisfazer $\operatorname{Ker}\left(T_{r}^{\overline{s(r)}}\right) \neq$ $\{0\}$.

Como $T_{r}^{\overline{s(r)}}(u, v, w)=(0,0,0)$ e a parte imaginária de $\overline{s(r)} \neq 0$ então a parte imaginária de $(u, v, w) \neq(0,0,0)$. Além disso, como todos os coeficientes de $T^{s(r)}$ são reais (exceto para $s(r)$ ), teríamos $T_{r}^{\overline{s(r)}}(\bar{u}, \bar{v}, \bar{w})=(0,0,0)$ e, portanto, teríamos pelo menos dois números, $s(r)$ e $\overline{s(r)}$ no conjunto

$$
\left\{s: \operatorname{Re} s>-\delta, \operatorname{Ker}\left(T_{r}^{s}\right) \neq\{0\}\right\} .
$$

De $(i)$ teríamos $s(r)$ e $\overline{s(r)}$ aproximando de 0 e portanto $\operatorname{dim}\left(W_{r}(0, \delta)\right) \geq 2$ o que contradiz $(i i)$. Isto nos mostra que $s(r) \in \mathbb{R}$ e é um autovalor de $L^{r}$, e pela Proposição $4.2 .1, s(r)<0$. 


\section{Capítulo 6}

\section{Resultados Numéricos}

Neste capítulo apresentamos alguns resultados numéricos para ilustrar o resultado principal do Capítulo 2.

\subsection{Definição da equação não local}

Tomando os parâmetros: $\varepsilon=0.01, \alpha=0.25, \gamma=20$ e a função $f(u)=u(u-0.25)(1-u)$, calculamos os valores $u_{2}$ e $F\left(u_{2}\right)$ cujas expressões são dadas por:

$$
u_{2}=\frac{1+\alpha}{2}+\frac{\sqrt{(1-\alpha)^{2}-\frac{4}{\gamma}}}{2} \text { e } F\left(u_{2}\right)=\int_{0}^{u_{2}} f(s) d s
$$

ou seja,

$$
u_{2}=0.9260 \text { e } F\left(u_{2}\right)=0.039842 .
$$

Com isso, temos a equação não local na forma

$$
\begin{aligned}
& u_{t}=u_{x x}-\lambda u_{x}+u(u-0.25)(1-u)-w \\
& w_{t}=-\lambda w_{x}+0.01(u-20 w)
\end{aligned}
$$

onde

$$
\lambda=\frac{0.039842-\left\langle w, u_{x}\right\rangle}{\left\langle u_{x}, u_{x}\right\rangle} .
$$

Consideramos ainda, as condições iniciais

$$
u(x, 0)=\frac{x+a}{2 a} \text { e } w(x, 0)=\frac{x+a}{40 a} \text { para }-a \leq x \leq a,
$$

e as condições de contorno

$$
u(-a, t)=0, \quad u(a, t)=0.9260, \quad w(-a, t)=0 \quad \text { e } \quad w(a, t)=0.0463 \quad \text { para } t>0 .
$$

\subsection{Resultados numéricos}

Para o sistema de FitzHugh-Nagumo, com o termo não liner da forma $f(u)=u(u-\alpha)(1-u)$, Gao e Wang (2004) mostrou que, para qualquer $\varepsilon>0$, se $\gamma>\frac{9}{(1-2 a)(2-s)}$ então a solução heteroclínica da equação

$$
\left\{\begin{aligned}
u^{\prime}(\xi) & =v(\xi) \\
v^{\prime}(\xi) & =c v(\xi)-f(u(\xi))+w(\xi) \\
w^{\prime}(\xi) & =\frac{\varepsilon}{c}(u(\xi)-\gamma w(\xi))
\end{aligned}\right.
$$


satisfazendo

$$
(u(-\infty), v(-\infty), w(-\infty))=(0,0,0) \quad \text { e } \quad(u(+\infty), v(+\infty), w(+\infty))=\left(u_{2}, 0, \frac{1}{\gamma} u_{2}\right)
$$

tem velocidade de propagação $c^{*} \in[\underline{c}, \bar{c})$, onde

$$
\underline{c}=\sqrt{2}\left(\frac{u_{2}}{2}-u_{1}\right) \text { e } \bar{c}=\sqrt{2}\left(\frac{1}{2}-a\right) .
$$

Com os parâmetros descritos na primeira seção deste capítulo, $\underline{c} \simeq 0,19$ e $\bar{c} \simeq 0,35$. Logo, $\lambda(t)$ deve convergir para um valor dentro do intervalo $[0,19,0,35)$.

De fato, usando solver ode15s do MATLAB, reformulamos o problema na forma

$$
\begin{aligned}
& u_{t}=u_{x x}-\lambda u_{x}+u(u-0.25)(1-u)-w, \\
& w_{t}=-\lambda w_{x}+0.01(u-20 w), \\
& \lambda\left\langle u_{x}, u_{x}\right\rangle-0.039842+\left\langle w, u_{x}\right\rangle=0, \\
& u(x, 0)=\frac{x+a}{2 a} \text { e } w(x, 0)=\frac{x+a}{40 a} \text { para } \quad u(a, t)=0.9260, \quad w(-a, t)=0 \quad w(a, t)=0.0463 \quad \text { para } t>0, \\
& u(-a, t)=0, \quad u(a, t)
\end{aligned}
$$

e tomando $a=40$, obtemos a velocidade de propagação

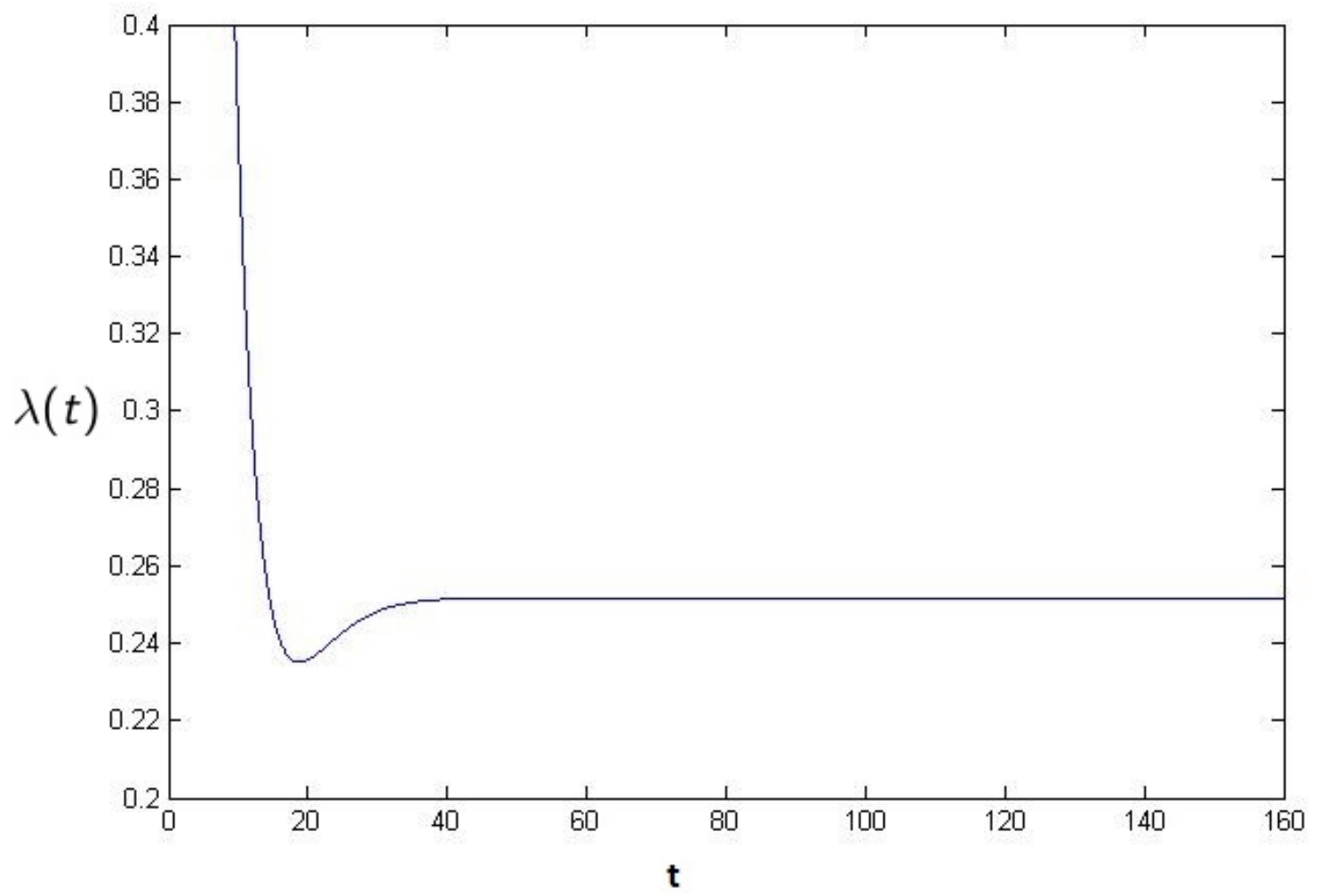

Figura 6.1: Velocidade de propagação $\lambda$

Da mesma forma, vemos que as soluções $u$ e $w$ convergem para o perfil de onda viajante 




Figura 6.2: Solução $u(x, t)$

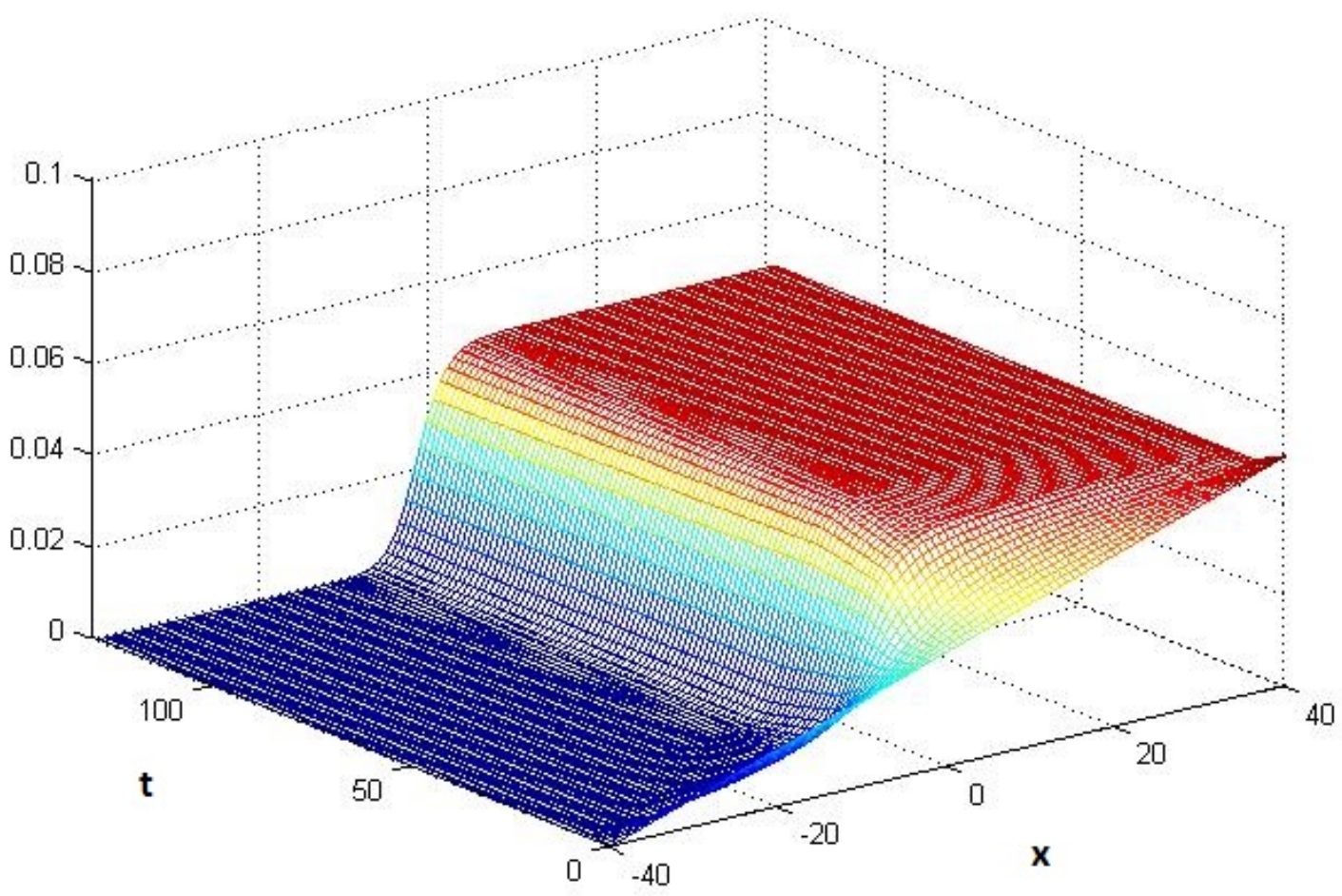

Figura 6.3: Solução $w(x, t)$ 
RESULTADOS NUMÉRICOS 


\section{Referências Bibliográficas}

Arrieta et al. (2011) Jose M Arrieta, Maria Lopez-Fernandez e Enrique Zuazua. Approximating travelling waves by equilibria of non local equations. arXiv preprint arXiv:1101.3454. Citado na pág. 37,41

Bates e Jones (1989) Peter W Bates e Christopher KRT Jones. Invariant manifolds for semilinear partial differential equations. Em Dynamics reported, páginas 1-38. Springer. Citado na pág. 13, 14, 15,16

Beyn e Lorenz (1999) Wolf-Jürgen Beyn e Jens Lorenz. Stability of traveling waves: dichotomies and eigenvalue conditions on finite intervals. Numerical Functional Analysis and Optimization, 20(3-4):201-244. Citado na pág. 50

Beyn e Rottmann-Matthes (2007) Wolf-Jürgen Beyn e Jens Rottmann-Matthes. Resolvent estimates for boundary value problems on large intervals via the theory of discrete approximations. Numerical functional analysis and optimization, 28(5-6):603-629. Citado na pág. 68

Beyn et al. (2014) Wolf-Jürgen Beyn, Denny Otten e Jens Rottmann-Matthes. Stability and computation of dynamic patterns in pdes. Em Current challenges in stability issues for numerical differential equations, páginas 89-172. Springer. Citado na pág. 37

Brezis (2010) Haim Brezis. Functional analysis, Sobolev spaces and partial differential equations. Springer Science \& Business Media. Citado na pág. 14

Budincevic (2010) Mirko Budincevic. A comparison theorem of differential equations. Novi Sad J. Math, 40(1):55-56. Citado na pág. 7

Coddington e Levinson (1955) Earl A Coddington e Norman Levinson. Theory of ordinary differential equations. Tata McGraw-Hill Education. Citado na pág. 19

Evans (1972) John W Evans. Nerve axon equations. i. linear approximations. Indiana Univ. Math. J, 21(9):877-885. Citado na pág. 13, 15

Evans (1975) John W Evans. Nerve axon equations. iv. the stable and unstable impulse. Indiana Univ. Math. J, 24(1169-1190):123-124. Citado na pág. 3, 22

Fife e McLeod (1977) Paul C Fife e J Bryce McLeod. The approach of solutions of nonlinear diffusion equations to travelling front solutions. Archive for Rational Mechanics and Analysis, 65 (4):335-361. Citado na pág. 5, 33

FitzHugh (1961) Richard FitzHugh. Impulses and physiological states in theoretical models of nerve membrane. Biophysical journal, 1(6):445. Citado na pág. 1

Friedman (2013) Avner Friedman. Partial differential equations of parabolic type. Courier Corporation. Citado na pág. 33

Gao e Wang (2004) Wenliang Gao e Jinghua Wang. Existence of wavefronts and impulses to fitzhugh-nagumo equations. Nonlinear Analysis: Theory, Methods \& Applications, 57(5):667-676. Citado na pág. 10, 73 
Ghazaryan et al. (2011) Anna Ghazaryan, Yuri Latushkin e Stephen Schecter. Stability of traveling waves for degenerate systems of reaction diffusion equations. Indiana Univ. Math. J, 60(2):443-472. Citado na pág. 15

Gohberg et al. (2012) Israel Gohberg, Seymour Goldberg e Marinus Kaashoek. Basic classes of linear operators. Birkhäuser. Citado na pág. 66

Hale (1980) Jack K. Hale. Ordinary Differential Equations. Robert E. Krieger Publishing Co. Citado na pág. 16

Henry (1981) Daniel Henry. Geometric theory of semilinear parabolic equations. Lecture Notes in Math, 840. Citado na pág. 3, 5, 15, 16, 20, 71

Hodgkin e Huxley (1952) Alan L Hodgkin e Andrew F Huxley. A quantitative description of membrane current and its application to conduction and excitation in nerve. The Journal of physiology, 117(4):500. Citado na pág. 1

Jones (1984) Christopher KRT Jones. Stability of the travelling wave solution of the fitzhughnagumo system. Transactions of the American Mathematical Society, 286(2):431-469. Citado na pág. $2,3,21$

Jones (1995) Christopher KRT Jones. Geometric singular perturbation theory. Em Dynamical systems, páginas 44-118. Springer. Citado na pág. 12

Kapitula e Promislow (2013) Todd Kapitula e Keith Promislow. Spectral and dynamical stability of nonlinear waves. Springer. Citado na pág. 21

Klaasen e Troy (1981) Gene A Klaasen e William C Troy. The stability of traveling wave front solutions of a reaction-diffusion system. SIAM Journal on Applied Mathematics, 41(1):145-167. Citado na pág. 30

Nagumo et al. (1962) Jinichi Nagumo, Suguru Arimoto e Shuji Yoshizawa. An active pulse transmission line simulating nerve axon. Proceedings of the IRE, 50(10):2061-2070. Citado na pág. 1

Oliveira (1992) Luiz A. F. de Oliveira. Existence of travelling waves in the fitzhugh-nagumo equation. Dynamic Systems and Applications, 1:83-92. Citado na pág. 2

Pao (2012) Chia-Ven Pao. Nonlinear parabolic and elliptic equations. Springer Science \& Business Media. Citado na pág. 39

Pazy (2012) Amnon Pazy. Semigroups of linear operators and applications to partial differential equations, volume 44. Springer Science \& Business Media. Citado na pág. 14

Pego (1985) Robert L Pego. Compactness in $l^{2}$ and the fourier transform. Proceedings of the American Mathematical Society, 95(2):252-254. Citado na pág. 16

Protter e Weinberger (2012) Murray H Protter e Hans F Weinberger. Maximum principles in differential equations. Springer Science \& Business Media. Citado na pág. 59

Rauch (1976) Jeffrey Rauch. Global existence for the fitzhugh-nagumo equations. Communications in Partial Differential Equations, 1(6):609-621. Citado na pág. 14, 39

Rauch e Smoller (1978) Jeffrey Rauch e Joel Smoller. Qualitative theory of the fitzhugh-nagumo equations. Advances in Mathematics, 27:12-44. Citado na pág. 13, 39, 41

Rothe (1984) Franz Rothe. Global solutions of reaction-diffusion systems. Citado na pág. 14, 39 
Rottmann-Matthes (2005) Jens Rottmann-Matthes. Spectral properties of mixed hyperbolicparabolic systems. Diplomathesis, Universität Bielefeld, Bielefeld. Citado na pág. 50, 51, 52, 53, 54

Sandstede (2002) Björn Sandstede. Stability of travelling waves. Handbook of dynamical systems, 2:983-1055. Citado na pág. 21

Smoller (1983) Joel Smoller. Shock waves and reaction - diffusion equations, volume 258. Springer Science \& Business Media. Citado na pág. 13

Sotomayor (1979) Jorge Sotomayor. Licões de equacões diferenciais ordinárias, volume 11. Instituto de Matemática Pura e Aplicada, CNPq. Citado na pág. 50

Szmolyan (1991) Peter Szmolyan. Transversal heteroclinic and homoclinic orbits in singular perturbation problems. Journal of differential equations, 92(2):252-281. Citado na pág. 2, 12

Tuma (1987) E Tuma. Comparison principles for strongly coupled reaction-diffusion equations. Proceedings of the Royal Society of Edinburgh: Section A Mathematics, 106(3-4):209-219. Citado na pág. 30

Tuma e Blázquez (1992) E Tuma e CM Blázquez. The stability of solutions in an initial-boundary reaction-diffusion system. Bulletin of the Australian Mathematical Society, 46(03):441-448. Citado na pág. 30

Vainikko (1979) Gennadii M Vainikko. Regular convergence of operators and the approximate solution of equations. Itogi Nauki i Tekhniki. Seriya"Matematicheskii Analiz", 16:5-53. Citado na pág. 66

Yanagida (1989) E Yanagida. Stability of travelling front solutions of the fitzhugh-nagumo equations. Mathematical and Computer Modelling, 12(3):289-301. Citado na pág. 3, 12, 15, 21, 22

Yanagida (1985) Eiji Yanagida. Stability of fast travelling pulse solutions of the fitzhugh — nagumo equations. Journal of Mathematical Biology, 22(1):81-104. Citado na pág. 2, 21 